$\rightarrow$ Schriften zum Kultur- und Museumsmanagement

Hartmut John (Hg.)

\title{
Shops und kommerzielle Warenangebote
}

\section{Publikumsorientierte Instrumente} zur Steigerung der Museumsattraktivität

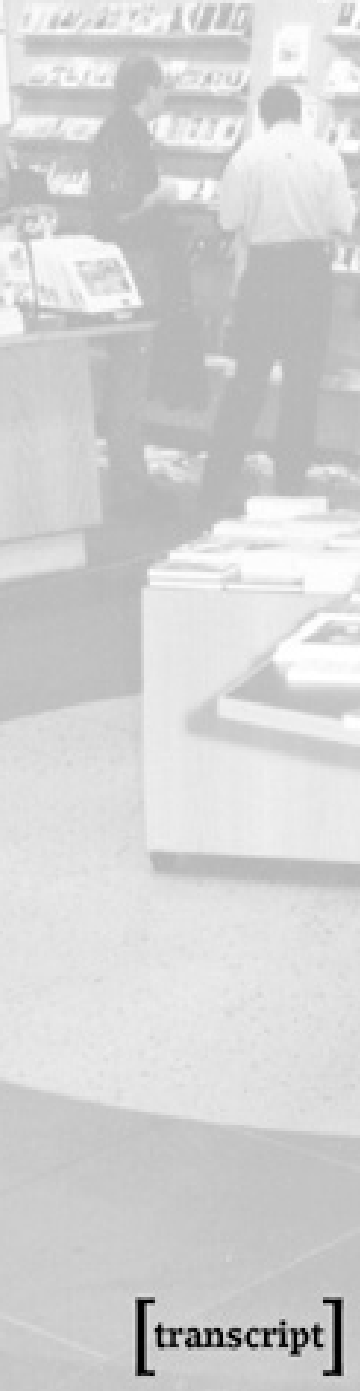


Shops und kommerzielle Warenangebote 
Publikation der Abteilung Museumsberatung Nr. 8

LANDSCHAFTSVERBAND RHEINLAND

Rheinisches Archiv- und Museumsamt 


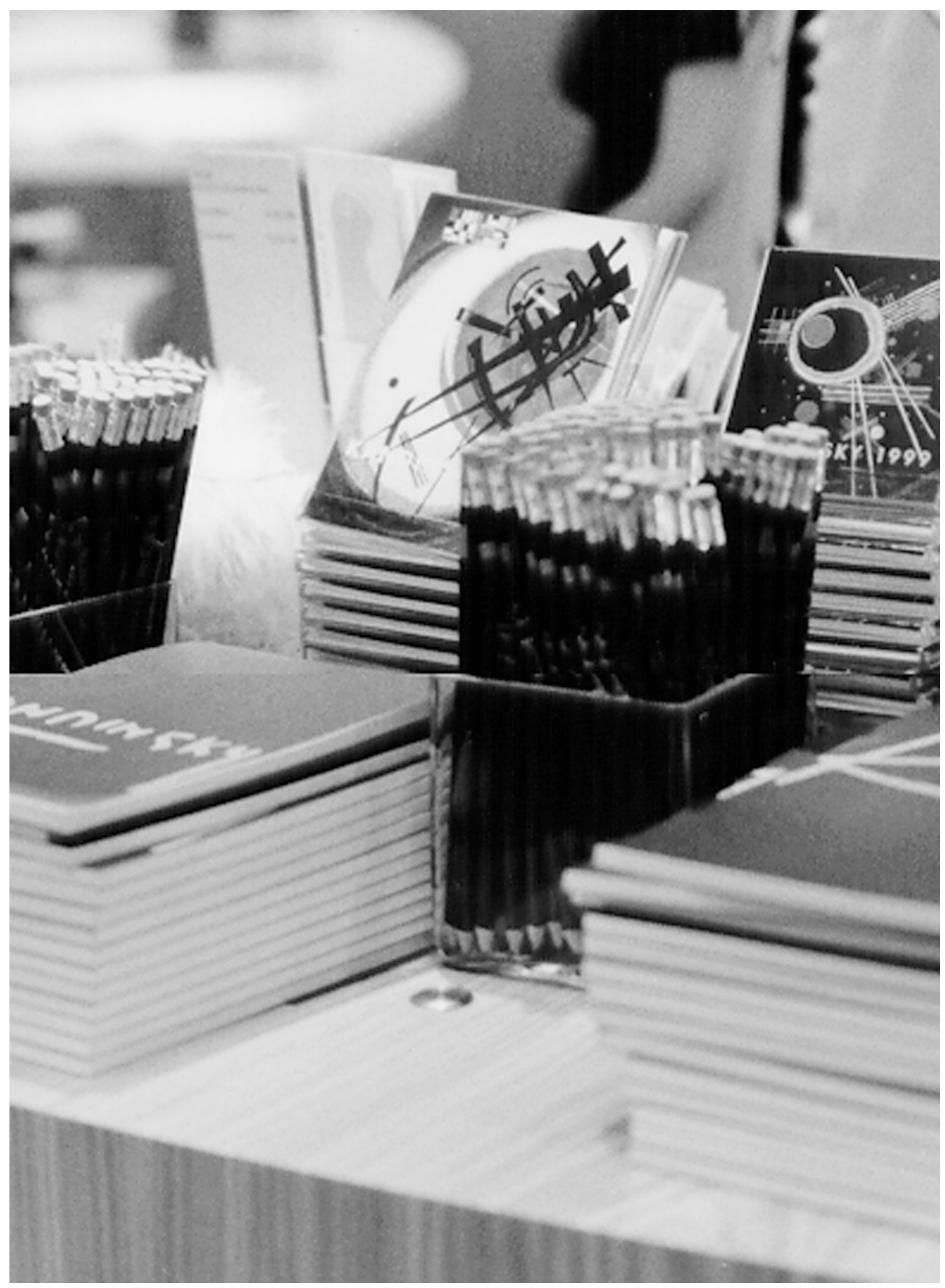



HARTMUt John (Hg.)

\title{
Shops und kommerzielle Warenangebote
}

\author{
Publikumsorientierte Instrumente \\ zur Steigerung der Museumsattraktivität
}

Publikation der Abteilung Museumsberatung Nr. 8

LANDSCHAFTSVERBAND RHEINLAND

Rheinisches Archiv- und Museumsamt 
Tagungsband zur gleichnamigen Veranstaltung

des Fortbildungszentrums Abtei Brauweiler/

Rheinisches Archiv- und Museumsamt

im Von der Heydt-Museum, Wuppertal,

am 17./18. September 1998

Herausgegeben von Hartmut John

im Auftrag des LANDSCHAFTSVERBANDES RHEINLAND

- Amt für Öffentlichkeitsarbeit -

- Rheinisches Archiv- und Museumsamt -

\section{(c) $\$($ This work is licensed under a Creative Commons \\ Attribution-NonCommercial-NoDerivatives 3.0 License.}

Die Deutsche Bibliothek -

CIP-Einheitsaufnahme

Shops und kommerzielle Warenangebote :

publikumsorientierte Instrumente zur Steigerung der Museumsattraktivität ; [Tagungsband zur gleichnamigen Veranstaltung des Fortbildungszentrums Abtei Brauweiler/Rheinisches Archiv- und Museumsamt im Von der HeydtMuseum, Wuppertal, am 17./ 18. September 1998] / Hartmut John (Hg.). [Im Auftr. des Landschaftsverbandes Rheinland, Amt für Öffentlichkeitsarbeit ; Rheinisches Archiv- und Museumsamt. Red. : ConCultura GmbH, Bonn]. -

Bielefeld : transcript Verlag, 2000

(Publikationen der Abteilung Museumsberatung ; Nr. 8)

(Schriften zum Kultur- und Museumsmanagement)

ISBN 3-933127-55-6

(C) 2000 transcript Verlag, Bielefeld

Landschaftsverband Rheinland und Autoren

Konzeption: Hartmut John

Organisation: ConCultura GmbH, Bonn

Redaktion: ConCultura $\mathrm{GmbH}$, Bonn

Umschlagfoto: Haus der Geschichte der

Bundesrepublik Deutschland, Bonn,

Fotostudio Querbach

Gestaltung: transcript Verlag, Bielefeld

Satz: digitron $\mathrm{GmbH}$, Bielefeld

Druck: Interpress, Budapest

Printed in Hungary

ISBN 3-933127-55-6 


\section{Inhalt}

HARTMUT JOHN

Vorwort

Hans WaLter HÜtTeR

Merchandising als Bestandteil eines integrierten

Museumskonzeptes

Haus der Geschichte der Bundesrepublik Deutschland

Eva HoffMeister

Museumsläden in NRW

Praktische Organisation und Marketingkennzahlen

BRIGITTE VoswiNKeL

Erfolgreiche Museumsshops

Anmerkungen zu Management und Produktentwicklung

BERND GÜNTER

Integration von Museumsshops in das Marketing-

konzept von Museen

Claus Peter Pithan

Steuerpflicht für Museumsshops 
Antonius J. Dommers

Organisations- und Trägerschaftsmodelle

für Museumsshops

Formen betriebswirtschaftlicher Ausgliederung

GABRIELE UELSBERG

Der Museumsshop in Trägerschaft eines

Fördervereins

ULRICH LÖBER

Die GmbH als Museumsshop-Betreiber

Autoren 
Beiträge 



\section{HARTMUT JoHN}

\section{Vorwort}

Auch hierzulande werden Musemsläden immer zahlreicher und sind aus vielen Museen nicht mehr wegzudenken. Bei Neu- oder Umbauten gehören Shops heute schon fast zur Regelausstattung. Inzwischen gibt es Shops, die den Anforderungen an einen modernen Kulturbetrieb in jeder Hinsicht genügen und betriebswirtschaftlich mit Erfolg geführt werden - bisweilen mit sechsstelligen Umsatzzahlen und diesen mehr oder weniger proportionalen Gewinn-Margen.

Dennoch: Trotz der zweifelsohne vorhandenen positiven Beispiele muß die deutsche Museumslandschaft insgesamt noch als "Entwicklungsland" in puncto Shops apostrophiert werden; und dies nicht nur gemessen am angelsächsischen Standard, der nicht in allen seinen Erscheinungsformen beispielgebend für unsere Kulturverhältnisse sein kann. Dementsprechend ist auch nicht weiter tragisch, daß Anglizismen wie "Kulturmerchandising", "Licensing « oder "Productplacement « und "Point of Sale " noch weithin Fremdworte in Museumskreisen sind. Allerdings hat die schwache Entwicklung der mit solchen Begriffen verbundenen Denk- und Verhaltensweisen negative Konsequenzen. Sie führt dazu, daß in vielen unserer Museen Läden und Verkaufsstellen geradezu vor den Besuchern versteckt werden. Peinliche Leere lädt zum schnellen Weitergehen ein: Außer Führern, Faltblättern und Postkarten gibt es häufig kaum etwas zu kaufen. Einige sogenannte Museumsshops sind von schlecht sortierten und präsen- 
tierten Buchläden nicht $\mathrm{zu}$ unterscheiden, andere sind leicht mit Souvenirläden oder Geschenkboutiquen zu verwechseln.

Interessanterweise hat sich auch das an den Hochschulen und Universitäten betriebene Kultur-/ Museumsmarketing kaum empirisch und analytisch mit der Funktion und dem Stellenwert von Verkaufsangeboten innerhalb des Museumsbetriebs auseinandergesetzt. Eine Ausnahme stellt der Lehrstuhl für Marketing um Prof. Dr. Bernd Günter an der Heinrich-Heine-Universität Düsseldorf dar, der in diesem Band die Ergebnisse seiner darauf bezogenen Arbeit vorstellt.

Dieser 'weiße Fleck im Museumsmarketing und die noch geringen Erfahrungen der Museen mit dem Verkaufen sind ursächlich für die in aller Regel überzogene Erwartung von Kulturpolitikern, Verwaltungs- und Museumsleuten, mit dem Betrieb von $\mathrm{Mu}$ seumsshops könnte man "so richtig Geld machen", um den Aufwanddeckungsgrad öffentlich subventionierter Museen entscheidend zu erhöhen. Unter dem Strich können selbst US-amerikanische Museen kaum mehr als ein Prozent ihrer Ausgaben aus Shopverkäufen finanzieren - beim New Yorker Metropolitan waren es 1995 zwei Prozent, und ähnlich ist die Situation beim Shop des Louvre (Paris).

Wichtiger als die Einnahmefunktion von Museumsläden für die Verringerung des öffentlichen Subventionsbedarfs ist ihre Rolle im Gesamtkontext der publikumsbezogenen Museumsangebote. Denn Verkaufsgeschäfte und -stellen im Museum können

- imageprägend und -fördernd für ein Museum sein, wenn Sortiment und Präsentation von entsprechender Qualität sind,

- Werbe- und Multiplikatoreneffekte für die Kultureinrichtung haben, Kundenbindungen stärken und die Zahl der Freunde und Förderer erhöhen,

- mittelbar Bildungsfunktion übernehmen, wenn die Verkaufsangebote auf das Museumsprofil ausgerichtet sind und an $\mathrm{Mu}-$ seumsthemen heranführen und schließlich

- einen wichtigen positiven Baustein innerhalb des »Gesamterlebnisses Museumsbesuch « darstellen, der die Zufriedenheit mit dem Museumsangebot positiv beeinflußt und die Bereitschaft wiederzukommen mitbestimmt. 
Wer in diesem Sinne Museen auch zu erlebnisreichen, anspruchsvollen Einkaufsorten entwickeln will, wer die Ausprägung einer >Merchandisingkultur in Museen als notwendig erachtet, wer Kommerz im Museum nicht, wie kürzlich die ZEIT, als Bedrohung der Museumsfundamente perhorresziert (Hanno Rauterberg in Nr. 14/1998), sollte sich mit dem Thema Museumsshops eingehend auseinandersetzen und im Detail befassen. Dabei möchte dieser Tagungsband des Fortbildungszentrums Abtei Brauweiler helfen. Allerdings nicht im Sinne eines 'Starterpackage`, einer >Rezeptsammlung، aus der Instant-Küche nach dem Motto: "Man nehme das richtige Produkt zum angemessenen Preis mit verkaufsfördernder Präsentation«; vielmehr ist es Anliegen dieser Publikation, einige erhellende Schneisen in den vielschichtigen, facettenreichen Gegenstand "Shops und kommerzielle Warenangebote für Museen« zu schlagen - von den strukturellen und organisatorischen Rahmenbedingungen des Betriebs von Museumsshops über Zielgruppenanalyse, Sortiment- und Preispolitik, bis hin zu Management-, Marketing- und Personalfragen. In Verbindung mit den vermittelten Erfahrungen und dem Know-how erfolgreicher Shop-Betreiber wollen sie dem Leser dabei helfen, Problemfelder und Sollbruchstellen schärfer zu erkennen und schon im Planungsstadium von Museumsläden mögliche Fallstricke und Fußangeln zu umgehen.

Natürlich bilden die vorgestellten Fallbeispiele und Modelle die Museumsshop-Realität nur teilweise ab. Sie dürfen deshalb auch nie 1:1 auf die Verhältnisse eines anderen Museums übertragen werden. Das Fortbildungszentrum hofft jedoch, mit dieser Publikation einen Beitrag zur Positionsklärung zu leisten und den Lesern so viel Orientierungs- und Problemwissen zu vermitteln, daß sie entsprechende Konzepte und Planungen für ihre Häuser kompetenter und zielgerichteter mitentwickeln und steuern können. Wir würden uns darüber hinaus freuen, wenn der vorgelegte Band der Diskussion darüber, wie Museen, Shops und kommerzielle Warenangebote zu publikumsorientierten Instrumenten für die Steigerung ihrer Attraktivität entwickeln können, Impulse geben könnte.

Allen Referenten und Autoren, dem gastgebenden Von der Heydt-Museum in Wuppertal und Frau Elke Hartkopf, M.A., vom Kulturberatungs-Unternehmen ConCultura in Bonn, die für die Tagungsorganisation und die Redaktion der Beiträge verantwortlich zeichnete, sei für Unterstützung und Engagement herzlich ge- 
dankt. Dem transcript Verlag in Bielefeld danken wir für die Aufnahme des Bandes in die Reihe der "Schriften zum Kultur- und Museumsmanagement«. 
HANS WALTER HüTteR

\title{
Merchandising als Bestandteil eines integrierten Museumskonzeptes
}

\author{
Das Haus der Geschichte der Bundesrepublik Deutschland
}

Der Besuch eines modernen, besucherfreundlichen und erlebnisorientierten Museums sollte nicht mit dem Ausstellungsrundgang enden, sondern im Idealfall erst im Museumsshop. Das - hoffentlich - positive Bild, das die Ausstellung geboten hat, sollte sich auch in den übrigen Serviceeinrichtungen eines Museums fortsetzen oder gar verstärken. Oft ist es insbesondere ein gut sortierter und das Kaufinteresse emotional weckender Museumsshop, der langfristig primäre Erinnerungen an Ausstellungsbesuche auslöst. Sich mit Inhalten, Themen und Exponaten des Museums vertieft auseinanderzusetzen, auch sich an den Museumsbesuch zu erinnern oder ein kleines Geschenk für Freunde und Familie mitzunehmen, sind die häufigsten Motive für die meist spontanen Käufe in Museumsshops. Eventshopping als Teil des Lebensgefühls hat für moderne, besucherorientierte Kultureinrichtungen hohe Bedeutung.

In Deutschland haben Verantwortliche in vielen Museen und Ausstellungshäusern bis heute noch Berührungsängste, in ihren Kultureinrichtungen kommerziell geführte Shops einzurichten. Die insbesondere aus den USA, aus Großbritannien und Frankreich sich zunehmend durchsetzenden Erfahrungen wie auch die immer knapper werdenden Mittel für die Durchführung operativer Aufgaben haben viele Kulturverantwortliche in jüngster Vergangenheit jedoch motiviert, sich intensiver mit Fragen des Merchandisings auseinanderzusetzen. 
Vor einem Irrglauben vieler Stadtkämmerer und Finanzminister muß jedoch sogleich gewarnt werden: Selbst vorzüglich organisierte und wirtschaftlich erfolgreich geführte Verkaufsstellen in Museen oder anderen Kultureinrichtungen können nicht dazu führen, daß die Träger sich aus der Finanzierung der Häuser zunehmend zurückziehen. In diesem Zusammenhang immer wieder angeführte Beispiele aus den Vereinigten Staaten von Amerika sind untauglich, undifferenziert auf deutsche Verhältnisse übertragen zu werden. Die Rahmenbedingungen in den USA sind völlig andere als in der Bundesrepublik Deutschland. Gewinne aus $\mathrm{Mu}-$ seumsshops und anderen Merchandisingaktivitäten können zwar helfen, das Budget von Museen und Ausstellungshäusern aufzubessern, sollen aber nicht allein oder vorrangig diese Aufgabe haben.

Ein gut geführter Museumsshop soll Bestandteil des Gesamtkonzeptes einer Kultureinrichtung sein. Die Entwicklung von museumseigenen Shopprodukten sowie das themenbezogene Angebot aus vorhandenen Sortimenten können gleichfalls zu Instrumenten der Öffentlichkeitsarbeit und Besucherbindung werden. Außerdem können Produkte dazu beitragen, den Bildungsauftrag der Kultureinrichtungen auch über den eigentlichen Aufenthalt im Haus hinaus weiter zu führen. Die Shopartikel können der themenbezogenen Nachbereitung, Vertiefung und so möglichst wieder zur Vorbereitung auf einen weiteren Besuch dienen.

In der Regel befinden sich Museumsshops unmittelbar im Museumsgebäude. Eine Reihe großer Museen, insbesondere in den USA und Großbritannien, haben Verkaufsstellen in Kaufhäusern, Shopping-Galerien, Bahnhöfen oder an anderen publikumsintensiven Stellen eingerichtet. Einige Museen bieten ihre Produkte auch über Versandkataloge an. Die wirtschaftlichen Erfolge dieser Aktivitäten außerhalb der Museen und Ausstellungshäuser sind abhängig von Produktangebot, Lage und Zielgruppe recht unterschiedlich. Beim Versandhandel haben einige Museen ihre Aktivitäten inzwischen wieder eingestellt, bei anderen boomt das Geschäft.

Die in Museen Verantwortlichen sollten die Möglichkeiten eines modernen Kulturmerchandisings intensiv und sorgfältig prüfen, sich freimachen von den immer wieder geäußerten Bedenken, Kultur dürfe keine kommerziellen Gedanken verfolgen, Kultur müsse frei bleiben von Kommerz, Kultur dürfe nur der Idee, dem Inhalt verpflichtet sein, Kultur stehe dem Kommerz geradezu kon- 
trär gegenüber. Diese Haltung, die insbesondere in Deutschland noch weit verbreitet scheint, da kulturelle Aktivitäten hier oft vollständig oder weitestgehend aus öffentlichen Mitteln finanziert werden, muten im internationalen Vergleich antiquiert an.

In den USA z. B. wurde bereits 1955 die Museum Store Association (MSA) mit Sitz in Denver gegründet. Diese Vereinigung hilft den Museen bei der Entwicklung und beim Vertrieb von Museumsshopprodukten. Sie bietet ferner ein umfangreiches Sortiment, aus dem Museen und andere Kultureinrichtungen schöpfen können, um mit nur geringem Aufwand ein attraktives Shopangebot kostengünstig und ohne hohes Risiko vorhalten zu können.

In Europa gibt es seit wenigen Jahren erste Versuche, Produzenten, Händler und Kultureinrichtungen zusammen zu bringen, umihre Ziele zu bündeln und die gemeinsamen Absichten kostengünstig und erfolgsorientiert umzusetzen. Die bisherigen Erfahrungen mit Museums-Messen in Deutschland, Frankreich und Großbritannien zeigen, daß die gewünschte wirtschaftlich orientierte, gemeinschaftliche Durchsetzung von Zielen bei weitem noch nicht erreicht ist. Einige Hersteller, z. B. von Kugelschreibern, haben inzwischen europaweit ansehnliche Marktanteile erreicht, indem sie Grundlagenprodukte entwickelt haben, die einer breiten Gruppe von Museen und Kultureinrichtungen, aber auch kommerziellen Unternehmen eine Basis bieten, kostengünstig individualisierte Produkte herzustellen.

Als während der achtziger Jahre der Neubau für das Haus der Geschichte der Bundesrepublik Deutschland in Bonn geplant und errichtet wurde, konnte in enger Abstimmung mit den Architekten des neuen Museumsgebäudes - Hartmut und Ingeborg Rüdiger aus Braunschweig - und den Verantwortlichen bei den Bundesbaubehörden ein zielgruppen- und sachgerechter Standort für den Museumsshop realisiert werden. Die infrastrukturellen Rahmenbedingungen orientieren sich an den Anforderungen des allgemeinen Einzelhandels. Wichtig ist, daß möglichst viele Besucher des Museums am Shop und dessen Auslagen vorbei geführt werden. Die Lage im räumlichen Gesamtkonzept, die Inneneinrichtung und die Präsentation der Produkte müssen dazu beitragen, die Besucher geradezu in den Shop hineinzusaugen. Durch den großzügigen Shopeingang im Foyer in unmittelbarer Anbindung an den Haupteingang des Museums wurde dieses Ziel erreicht. Zusätzliche Auslagen vor dem Shopeingang im Bereich des Foyers, ein Informationsterminal für die hauseigene CD-ROM »Erlebnis Geschichte. Deutschland seit 1945" sowie Leuchtreklamen 
helfen, den Aufmerksamkeitswert des Museumsshops noch zu steigern.

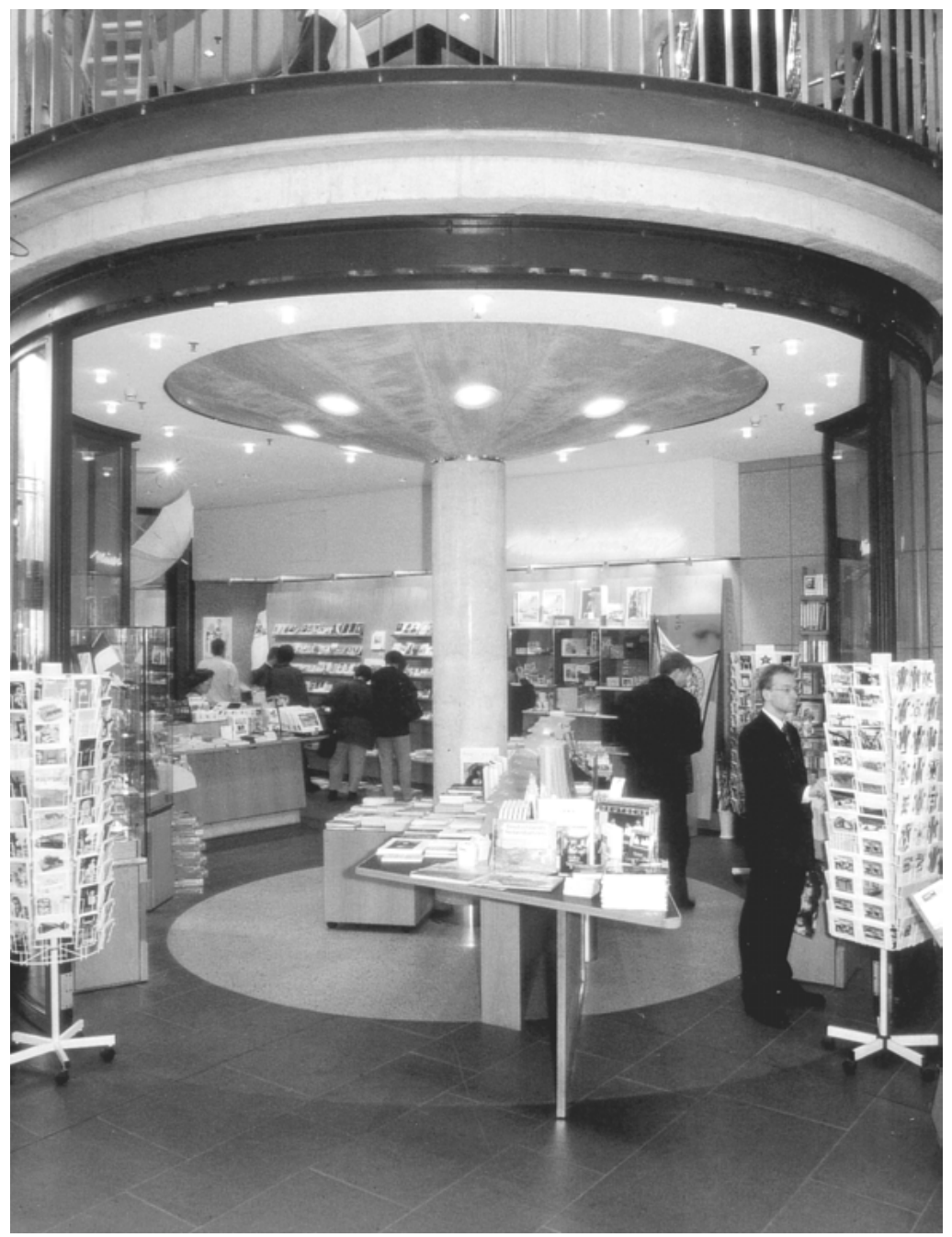

Lösungen, die sich für das eine Haus mit seinen Rahmenbedingungen als tragfähig oder gar optimal erweisen, sind auf eine andere Einrichtung nicht unbedingt erfolgversprechend übertragbar. Jeder einzelne Betreiber muß gemessen an seiner individuellen Zielgruppe, an den Themen, den infrastrukturellen und finanziellen Möglichkeiten orientierte Individuallösungen finden. 
Um ein erfolgsorientiertes Kulturmerchandising $\mathrm{zu}$ entwickeln, sind folgende Fragestellungen hilfreich:

- Welche Zielgruppen erreichen meinen Shop ohnehin, welche kann ich durch gezieltes Merchandising zusätzlich akquirieren und binden?

- Welche Produkte will und kann ich themen- bzw. ereignisbezogen anbieten?

- Welche infrastrukturellen und räumlichen Möglichkeiten stehen mir zur Verfügung?

- Welche personellen Möglichkeiten habe ich?

- Welche Organisationsform bietet sich an?

\section{Zielgruppen}

In jeder Kultureinrichtung - übrigens auch bei jedem Einzelhandelsgeschäft - ist zunächst zu klären, welche Zielgruppen das Museum anspricht oder künftig ansprechen will, welche das Haus und damit den Museumsshop ohnehin erreichen und wie diese durch gezielte Werbung und Angebote zusätzliche gebunden werden können.

Für den Museumsshop im Haus der Geschichte konnten bis zur Eröffnung des Museums im Juni 1994 im Rahmen des Gesamtkonzeptes zunächst nur Ziele und Wünsche formuliert werden. Nach fast fünf Jahren Erfahrung lassen sich Zielgruppen und Kaufverhalten aufgrund umfänglicher statistischer Erhebungen und Besucherbefragungen recht exakt analysieren.

Das Haus der Geschichte präsentiert besucherfreundlich und erlebnisorientiert deutsche Zeitgeschichte vom Ende des Zweiten Weltkrieges bis in die Gegenwart. In seiner Grundidee war es von Beginn an darauf ausgerichtet, breite Bevölkerungsschichten, den >Alltagsmenschen`, zu erreichen, nicht vorrangig den spezialisierten >Museumsmenschen $`$ anzusprechen. Die dezidierte Zielgruppenprognose vor Eröffnung des Museums und die inzwischen vorliegenden statistischen Untersuchungen sind Grundlage für die Zusammenstellung des Shopsortimentes. Hierzu gehören vor allem Literatur und diverse Non-Book-Produkte, die sich themenbezogen bereits auf dem Markt befinden sowie die Entwicklung und Produktion eigener, allein im Shop des Hauses der Geschichte vertriebener Artikel. Nach Auswertung der Besucherdaten seit Er- 
öffnung des Hauses bestätigt sich die Prognose, daß für eine breite Kundenschicht ein vielfältiges Sortiment anzubieten sinnvoll ist. Hierbei ist insbesondere $\mathrm{zu}$ berücksichtigen, daß Gruppenbesucher ein erheblich von Individualbesuchern abweichendes Kaufverhalten an den Tag legen.

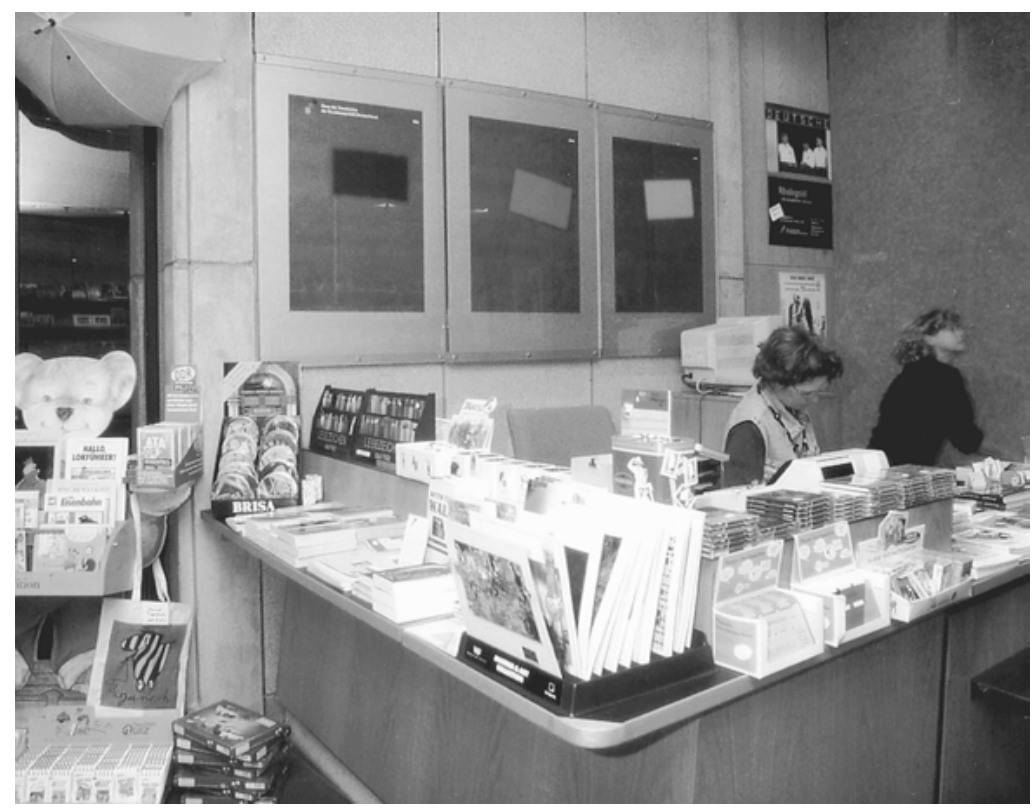

Der Anteil der Museumsbesucher, die auch Kunden des Museumsshops werden, ist ein Indikator für die Attraktivität des Shopangebotes. Gleichwohl muß das traditionell unterschiedliche Kaufverhalten bei Museumsbesuchern im In- und Ausland berücksichtigt werden. 20 bis 30 Prozent der Museumsbesucher in traditionsreichen und international angesehenen Museen in London werden auch Shopkunden. Demgegenüber stehen fünf bis zehn Prozent der Gesamtbesucher in gut ausgestatteten Museumsshops in Deutschland. Diese Anteile sinken bei steigender Gruppenfrequenz und Gesamtbesucherzahl auf bis zu 0,5 Prozent der Gesamtbesuche. Nach Optimierung der Präsentation sowie des Shopangebotes in den vergangenen vier Jahren seit seiner Eröffnung erreicht das Haus der Geschichte mittlerweile zwischen acht und zehn Prozent der Besucher der Dauerausstellung auch als Shopkunden. 
Shopbetreiber im Kulturbereich sollten berücksichtigen, ob Ausstellungen und Veranstaltungen regionalen Bezug haben und somit die Bevölkerung der engeren Umgebung einbeziehen oder ob die regionale Wohnbevölkerung von geringerer Bedeutung ist, da die Ausstellungen von nationalem oder internationalem Rang eher von Menschen aus der weiteren Umgebung besucht werden. Gäste, die mehr unter touristischen Aspekten Ausstellungen besuchen, sind eher bereit, für Shopprodukte auch höhere Beträge zu investieren, als das Publikum, das zum »Museum um die Ecke» kommt. Stammpublikum ist hingegen von besonderem Interesse beim Aufbau eines Fachsortimentes. Der Museumsshop kann auf diese Weise zum Buchladen und Geschenkshop für Dauerkunden werden, die insbesondere in kleineren Einrichtungen einen erheblichen Teil des Umsatzes ausmachen können. Bei der Untersuchung der Besucherzahlen ist eine detaillierte Analyse notwendig, um eine solide Grundlage für die Bewertung von Umsätzen, Umsatzrelationen, Produktakzeptanz etc. zu ermitteln. Nur differenzierte Auswertungen können konkrete Hinweise geben, um das Sortiment zielgruppengerecht auszurichten.

\section{Produkte}

Grundsätzlich gelten für Produktangebote im Umfeld kultureller Aktivitäten, d.h. auch in Museen und Ausstellungshäusern, die allseits bekannten Gesetze des Marktes. Die Produkte stehen in harter Konkurrenz zu den Angeboten außerhalb der Kulturbetriebe. Attraktive und qualitativ hochwertige Produkte zu akzeptablen Preisen sind Grundvoraussetzungen für den Verkaufserfolg. Die Chance des Kulturmerchandisings besteht vor allem darin, an besonderem Ort in ereignisbezogener, emotionaler Situation Produkte anzubieten, die in engem Zusammenhang mit einem positiv besetzten Erlebnis stehen. Der Museums- oder Ausstellungsbesucher ist unmittelbar während oder nach dem Ausstellungsrundgang besonders eingestimmt, so daß sich das Angebot im inhaltlichen Umfeld bewegen sollte. Jetzt - und nicht Wochen später - ist der Besucher motiviert, sich mit Produkten, die in enger Verbindung mit der Museums- oder Ausstellungsthematik stehen, zu beschäftigen. Hierbei sollten die Artikel einen Bezug zum Museum, zum Ereignis oder dessen Themenumfeld haben. Wenn die Produkte Informationen über Museum oder Ausstellung vermitteln, 
erreichen sie gleichzeitig einen wünschenswerten Werbe- und Multiplikatoreneffekt.

Bei der Zusammenstellung des Produktangebotes für den $\mathrm{Mu}$ seumsshop können entweder auf dem Markt vorhandene Artikel übernommen, ggf. durch Verpackung, Aufschrift o. ä. individualisiert, gemeinsam mit dem Anbieter neue Produkte entwickelt oder in alleiniger Verantwortung des Museums Artikel hergestellt werden. Meist werden die finanziellen Möglichkeiten die Grenzen eigener Produktentwicklung aufzeigen.

Im Haus der Geschichte wurde vor der Eröffnung auf der Basis der antizipierten Besucherstruktur in enger Zusammenarbeit zwischen dem Museum und dem Pächter des Museumsshops - dem Geschäftsführer der Rheinland-Verlag- und Betriebsgesellschaft (RVBG) - das Produktsortiment für den Shop zusammengestellt. Seither werden die Artikel regelmäßig ergänzt, aktualisiert, zum Teil auch reduziert.

Eng angelehnt an die Themen der Ausstellungen des Hauses ist ein wesentlicher Bestandteil des Shopsortimentes im Museum für Zeitgeschichte in Bonn zeithistorische und politikwissenschaftliche Literatur. Wissenschaftliche Werke, auch populär-historische Darstellungen, Karikaturen- und Bildbände, Lexika, kindund jugendgerechte Fachliteratur wurden themenspezifisch ausgewählt und können aus den umfangreichen Verlagsangeboten jederzeit ergänzt werden. Hierdurch wurde der Museumsshop zu einem Fachbuchhandel zur deutschen Geschichte seit 1945, in dem die Kunden eine in üblichen Buchhandlungen nicht vorhandene Breite und Tiefe themenspezifischer Publikationen finden. In jüngster Zeit treten zunehmend auch elektronische Medien zum Thema - CDs und CD-ROMs - in das Blickfeld der Museumsbesucher.

Die selbstentwickelten Produkte - vor allem bezogen auf die Sammlungen und Ausstellungen des Hauses - geben dem Shop im Haus der Geschichte sein individuelles Gesicht. Für jede Wechselausstellung des Hauses erscheint gemeinhin eine eigene Publikation, meist ein reich illustriertes Begleitbuch, ein Magazin oder eine Broschüre. Diese Publikationen werden in der Regel auch im Buchhandel bundesweit angeboten, so daß der gewünschte PR-Effekt im Rahmen des integrierten Museumskonzeptes erreicht wird. Ferner ist so die Gesamtauflage zu steigern und der Abgabepreis im Museumsshop zu reduzieren, gleichzeitig die Gewinnspanne für das Museum zu erhöhen. Von besonderem Interesse ist das 
Buch zur Dauerausstellung »Erlebnis Geschichte«: Reich illustriert und allgemein verständlich formuliert, ist diese Publikation zum Museumsshoppreis von 39 DM (im Buchhandel 58 DM) für die Zielgruppe des Museums ein neuartiges Buch zur deutschen Geschichte seit Ende des Zweiten Weltkrieges. Hiervon verkauft der Museumsshop jährlich ca. 10.000 Exemplare. Die inzwischen erschienene zweite, aktualisierte Auflage und die folgenden Editionen sind trotz gleichbleibenden Verkaufspreises von besonderem wirtschaftlichen Interesse.

Mit der Publikationsreihe »Objekte im Museum» beschreitet das Haus der Geschichte einen neuen Weg. In thematischhistorischem Zusammenhang werden herausragende Ausstellungsobjekte oder Objektgruppen erläutert. Das Exponat in der Ausstellung ist der visuell-gedankliche Impuls, die Broschüre wird anschließend zur griffigen Erinnerung, zum persönlichen Mitnahmeexemplar. Die attraktiv gestalteten Publikationen werden fortlaufend ergänzt und exklusiv im Museumsshop für 9,80 DM angeboten. Die Entwicklung und Realisierung dieser Publikationsreihe ist außerdem ein gutes Beispiel für eine enge und erfolgreiche Kooperation mit einer Agentur, die sich u.a. auf Ziele und Aufgaben von Kultureinrichtungen spezialisiert hat: Das Haus der Geschichte liefert Texte und eine vorrecherchierte Fotoauswahl, nach grundsätzlicher Gestaltungsentscheidung realisiert die Agentur Schleiner + Partner in Freiburg diese Publikationen zu einem vorab ausgemachten Preis bis zur Anlieferung im Shop.

Eine Publikation besonderer Art ist die CD-ROM »Erlebnis Geschichte. Deutschland seit 1945", die das Haus der Geschichte gemeinsam mit dem Ernst Klett Verlag in Stuttgart und den TC Studios in Ludwigsburg entwickelte und produzierte. Diese multimediale, animative CD-ROM mit zahlreichen Ton- und Filmdokumenten, Informationstexten, Fotos, Statistiken etc. kann der $\mathrm{Mu}-$ seumsbesucher zum Museumsshoppreis von 89 DM (im Buchhandel für 98 DM) erwerben. Der Verkauf der in vollem Umfang von den privaten Partnern vorfinanzierten CD-ROM entspricht den Erwartungen, die zweite Fassung konnte bereits ein Jahr nach der öffentlichen Präsentation hergestellt werden. Ein exklusiv für diese CD-ROM im Foyer des Museums eingerichteter Informationsterminal in enger Anbindung an den Museumsshop animiert die Besucher, sich auch auf diese multimediale Form mit der deutschen Zeitgeschichte zu beschäftigen, gleichzeitig erhalten sie den 
Hinweis, daß sie nebenan im Shop dieses Produkt zum Sonderpreis erwerben können.

Der Museumsshop im Haus der Geschichte erzielt mit den Publikationen aus den Verlagssortimenten und den eigenen ca. zwei Drittel des Gesamtumsatzes. Print- und printverwandte Produkte spielen im Museumsshop des zeithistorischen Museums eine erhebliche - auch wirtschaftliche - Rolle.

30 Prozent des Gesamtumsatzes erzielt der Museumsshop im Haus der Geschichte mit eigens erstellten Non-Book-Artikeln. Hierbei erweisen sich Postkarten als besonders zugkräftig. Dauerhaft attraktiv sind auch die angebotenen Modellautos im HO-Maßstab 1:87. Die in der Dauerausstellung des Museums gezeigten Fahrzeuge werden in einer dem Corporate Design des Hauses entsprechenden Verpackung mit historischer Erläuterung angeboten. Die Realisierung erfolgt mit verschiedenen namhaften deutschen Herstellern.

In Kooperation mit dem Museumsshoppächter entstehen anläßlich jeder neuen Wechselausstellung themen-, zielgruppenund jahreszeitlich orientierte Produkte. Initiativen und Vorschläge kommen sowohl vom Shoppächter wie auch von den Projektgruppen aus dem Haus. Nach gemeinsamer Prüfung fällt dann im Einvernehmen die Entscheidung für die jeweils $\mathrm{zu}$ realisierenden Produkte, die teils vom Shoppächter, teils vom Museum vorfinanziert werden.

Das Sortiment ist regelmäßig und in kurzen Abständen zu durchforsten. Neben dem Grundsortiment an Book- und NonBook-Artikeln verlieren vor allem die anläßlich von Wechselausstellungen realisierten Produkte nach recht kurzer Zeit an Bedeutung. Daher sollte bei der Realisierung eigener Artikel darauf geachtet werden, daß deren Bezug zum Museum auch außerhalb der engen Wechselausstellungsgrenzen möglich ist. Durch exakte Analyse der Produktpalette, der Verkaufszahlen und des sich wandelnden Käuferverhaltens ist eine solide Basis für die Aktualisierung des Shopsortimentes zu gewinnen, dessen Straffung in regelmäßigen Abständen wünschenswert ist.

\section{Produktpräsentation}

Einkauf gilt dem Lebensnotwendigen, Shopping eher der Lebensfreude! Dabei wird die Warenpräsentation zum visuellen Kaufer- 
lebnis. Mindestens so attraktiv wie die Ausstellung muß das Angebot im Museumsshop sein. Kunden und Käufer müssen sich wohl fühlen, zum Schnuppern und Stöbern angeregt werden. Die Präsentation der Produkte muß in regelmäßigen Abständen neu organisiert werden. Mit Blick auf besondere Ereignisse, Jahrestage, Ausstellungsthemen o. ä. müssen die Artikel leicht auffindbar und attraktiv präsentiert werden. „Crossmerchandising "kommt dem emotionalen, Event-bezogenen Einkaufsverhalten entgegen.

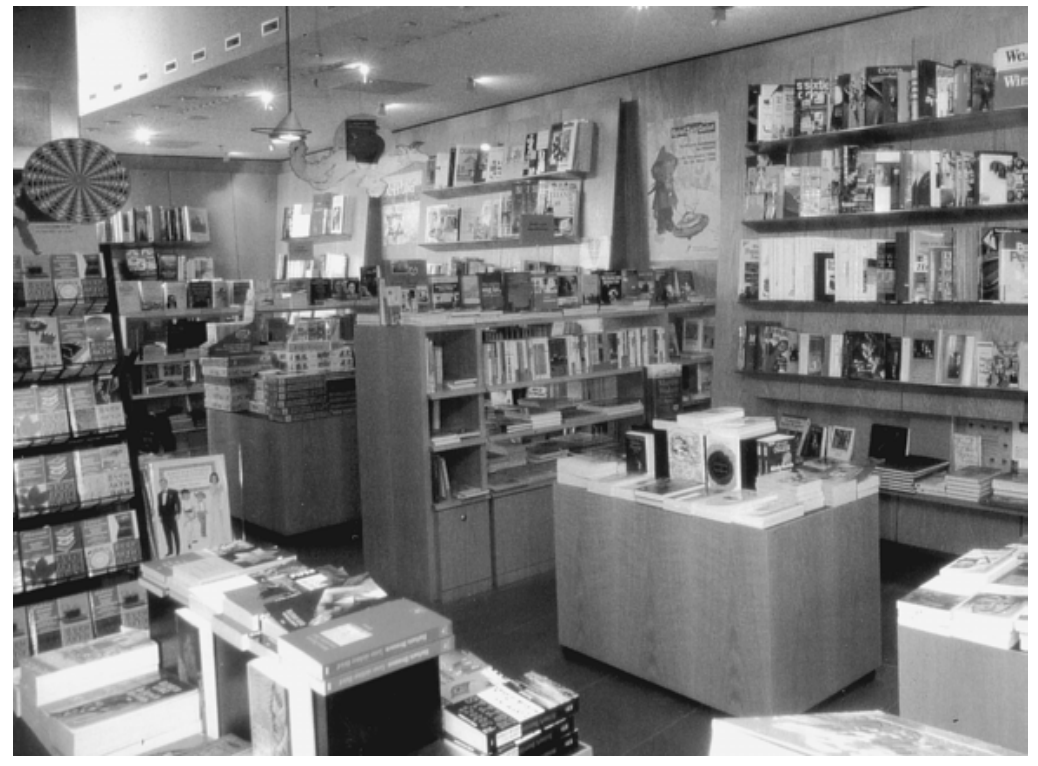

Im Haus der Geschichte der Bundesrepublik Deutschland hat der Museumsshop bereits seit Beginn der Planungen unmittelbar am Haupteingang des Hauses eine kundengünstige Lage. Großzügig verglaste Flächen an der Außenfassade und zum Foyer hin entsprechen der architektonischen Gesamtidee des neuen Museumsgebäudes, transparent und offen den Besucher anzusprechen. Die architektonischen Vorgaben des Gebäudes erlauben eine Zweiteilung des Shops. Im hinteren, ruhigeren Bereich sind vor allem die Publikationen, im vorderen, unmittelbar zum Foyer hin gelegenen offeneren Teil vorrangig das Non-Book- und Produktsortiment des Museums präsentiert. Die Besucher, die sich in Ruhe mit der Auswahl von Fachliteratur beschäftigen wollen, werden durch Käufer nicht gestört, die lediglich ein Souvenir erwerben wollen. An der Nahtstelle zwischen beiden Shopbereichen steht die Verkaufs- 
theke mit elektronischer Kasse, die auch kreditkartentauglich ist. So erfüllt diese Aufteilung einen kostengünstigen Personaleinsatz und recht gute Kontrollmöglichkeiten.

Die Inneneinrichtung des Museumsshops wurde in Absprache zwischen Museum und Architekten des Hauses ausgewählt und gestaltet. Bei den konkreten Präsentationen von Produkten und Artikelgruppen wirkten der Museumsshoppächter, die Leiterin des Shops und die im Museum Verantwortlichen intensiv zusammen. Nach ca. zwei Jahren Betriebsphase konnten aufgrund der Beobachtungen und statistischen Ergebnisse ohne großen Aufwand weitere Verbesserungen bei den Präsentationsmöglichkeiten erreicht werden.

Ein konsequent themenbezogenes Produktangebot - ggf. an anderer Stelle zusätzlich durch die Darbietung im Rahmen der Produktgattung ergänzt - ist käuferfreundlich und umsatzfördernd. Die Produkte zu jeweiligen Wechselausstellungen bietet der Museumsshop im Haus der Geschichte an zentraler Stelle an: Begleitpublikation, ausgewählte, handelsübliche Fachliteratur und selbstentwickelte Merchandisingprodukte. An anderen Stellen im Shop, an denen z. B. Fachliteratur oder kindgerechte Artikel angeboten werden, sind die zum Thema präsentierten Produkte, falls dies sinnvoll erscheint, erneut vorhanden.

Schließlich sollte eine gezielte, Synergieeffekte nutzende Werbung die Präsentation von Shopartikeln unterstützen. Im Haus der Geschichte schaltet der Pächter des Shops regelmäßig Anzeigen mit aktuellen Produktangeboten im Museumsmagazin. Diese Werbeanzeigen werden ergänzt durch Vorstellungen neuer Produkte, Publikationsrezensionen etc. Bei jeder Pressekonferenz und Eröffnung einer Wechselausstellung im Haus der Geschichte bilden auch die neuen und aktuellen Shopprodukte einen Schwerpunkt.

\section{Infrastruktur}

Die Infrastruktur eines Museumsshops muß unbedingt auf die erwarteten Käufergruppen und -zahlen ausgerichtet sein. Nicht die Größe der Verkaufsfläche ist bei der Einrichtung einer Verkaufsstelle in Kultureinrichtungen entscheidend, sondern vielmehr die Lage und die geschickte Präsentation attraktiver Produkte. Hingegen können überdimensionierte Verkaufsstellen den wirtschaftlichen Gewinn erheblich schmälern. 


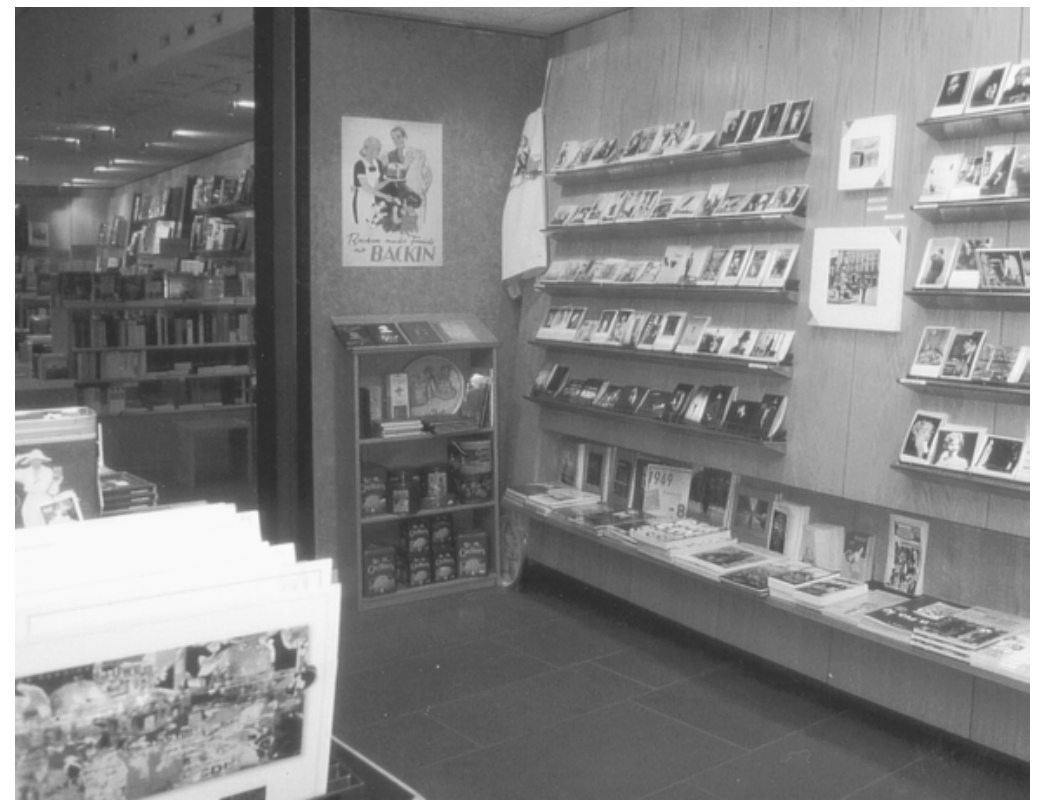

$\mathrm{Zu}$ prüfen ist auch, in welcher Form der Shop geführt wird. Einheitslösungen können bei der Vielfalt der kulturellen Einrichtungen und individuellen Rahmenbedingungen nicht erfolgversprechend sein. Ob der Shop im Eigenbetrieb mit Freiwilligen oder Teilzeitbeschäftigten, mit einem Alleinpächter oder einer Betriebsgesellschaft, die auch andere Bereiche des Hauses organisiert, oder gar als Einrichtung des Museums selbst geführt wird, ist von den individuellen Möglichkeiten und Zielsetzungen abhängig.

Beim Haus der Geschichte in Bonn zeichnete sich bereits frühzeitig vor der Eröffnung ab, daß die Stiftung selbst weder Zeit noch Personal haben würde, den Museumsshop in Eigenregie zu führen. Nach zahlreichen intensiven Gesprächen und einer Ausschreibung fiel die Entscheidung, den Museumsshop an die Rheinland-Verlag- und Betriebsgesellschaft zu verpachten. Diese Gesellschaft ist eine $\mathrm{GmbH}$ in Trägerschaft des Landschaftsverbandes Rheinland. Insgesamt betreut die RVBG inzwischen mehr als 25 Museen in unterschiedlichem Umfang.

Auswahl, Aus- und Fortbildung des Verkaufspersonals für den Museumsshop ist ein wesentlicher Baustein zum Erfolg. Ob freiwillig, ehren- oder hauptamtlich tätiges Museumsshoppersonal, in jedem Fall sollte sichergestellt sein, daß die Mitarbeiterinnen und 
Mitarbeiter sich in hohem Maße mit dem Haus und den Produkten identifizieren und daß sie motiviert sind, sich weit über das im Einzelhandel übliche Verkaufsgespräch hinaus zu engagieren. Nur so ist weitgehend sicherzustellen, daß der Museumsbesucher und Shopkunde optimal beraten wird und sich hieraus für das Haus auch ein wirtschaftlicher Erfolg einstellt.

Auch qualifiziertes und motiviertes Museumsshoppersonal muß aus- und fortgebildet werden. Wichtig ist hierfür ein regelmäßiger Gedankenaustausch über Ziele und Programm des Museums, über Erfahrungen mit Museums- und Museumsshopbesuchern etc.

Im Haus der Geschichte arbeiten der Museumsshoppächter sowie die Museumsleitung auch in Fragen der Personalauswahl, Aus- sowie Fortbildung intensiv und vertrauensvoll zusammen übrigens weit über das vertraglich vereinbarte Maß hinaus. Die personelle Kontinuität im Museumsshop, die Qualität der Beratung, das persönliche Engagement auch außerhalb üblicher Öffnungszeiten und nicht zuletzt der wirtschaftliche Erfolg stellen diesem Kooperationsmodell ein gutes Zeugnis aus.

Schließlich noch ein Blick auf die haushaltsrechtlichen Bedingungen in Deutschland: Oft ist es im Rahmen des öffentlichen Haushaltsrechtes problematisch, die durch Produktverkauf erzielten Gewinne wieder unmittelbar für Zwecke des Museums einzusetzen. In vielen Fällen fließen erzielte Gewinne dem Träger, nicht aber dem Betreiber wieder $\mathrm{zu}$, wodurch die Motivation, durch Merchandising Einnahmen und Gewinne zu erzielen, naturgemäß gegen Null tendiert. Wenn hingegen die erzielten Gewinne den Einrichtungen wieder unmittelbar zu Gute kommen, ist es für das Museum auch sinnvoll, den Shop selber zu betreiben. In vielen Fällen wählen Museen den Weg, eine Betriebsgesellschaft zu gründen, die freier von haushaltsrechtlichen Vorschriften nach den Gesetzen des Marktes operieren kann. In anderen Fällen betreiben Förder- und Freundeskreise auf privatrechtlicher Basis die Verkaufsstellen in Kultureinrichtungen. In jedem Einzelfall ist die betriebswirtschaftlich günstigste Lösung zu suchen, die mit dem jeweiligen Haushaltsrecht des Trägers und den Steuergesetzen in Einklang steht. Die inzwischen zunehmend praktizierte Budgetierung von Kultur- und Bildungseinrichtungen in öffentlicher Trägerschaft wird künftig die Arbeit der Häuser erleichtern. Bei allen Betriebsformen ist jedoch zu beachten, daß langfristig solide, auf partnerschaftlicher Basis beruhende Lösungen in aller Regel er- 
folgversprechender sind als vordergründig nur auf Gewinnmaximierung ausgerichtete Vertragsverhältnisse.

Die wirtschaftlichen Ergebnisse, die seit Eröffnung des Hauses der Geschichte der Bundesrepublik Deutschland im Juni 1994 in Bonn erzielt wurden, lassen eine positive Bewertung der Zielgruppenanalyse, der Auswahl des Produktsortimentes, der Infrastruktur und der Einkaufs- und Abgabepreise zu. Seit dem Eröffnungsjahr konnten die Umsatzergebnisse zum Teil erheblich gesteigert werden, obwohl die Besucherzahlen - naturgemäß - im ersten Jahr nach der Eröffnung am höchsten waren. Ein Beispiel: Im besucherstarken Oktober wurde der Umsatz von 1994 bis 1998 kontinuierlich um insgesamt mehr als 40 Prozent gesteigert. Durchschnittlich ist im Museumsshop des Hauses der Geschichte in Bonn seit 1995 eine Umsatzsteigerung von mehr als 20 Prozent zu verbuchen. Die Transaktionswerte pro Käufer bewegen sich um 20 DM. Gemessen an den Gesamtbesuchen in der Dauerausstellung gibt jeder Besucher im Museumsshop inzwischen ca. 1,6o DM aus. 1996 lag dieser Wert noch bei 1,30 DM. Verglichen mit z.B. international renommierten Kunstmuseen in den USA oder Großbritannien, die einen sehr hohen Anteil touristischen Publikums analysieren können, sind diese Werte noch steigerungsfähig. Doch bei Betrachtung historischer, technik- und kulturhistorischer Museen mit breiter Zielgruppe in Deutschland hält der Museumsshop im Haus der Geschichte einen der vorderen Plätze. Verglichen mit gut florierenden Buchhandlungen kann der Shop, bezogen auf Verkaufsfläche, Personal- und Wareneinsatz, gute Werte vorweisen.

Bei der Einrichtung einer Verkaufsstelle im Zeitgeschichtlichen Forum Leipzig der Stiftung Haus der Geschichte der Bundesrepublik Deutschland, das im Herbst 1999 eröffnet wurde, sind die Erfahrungen und Erkenntnisse bei der Vorbereitung und Führung des Shops in Bonn nur mit erheblichen Variationen zu übertragen. Durch die architektonisch bedingte andere Lage des Shops, das antizipierte zum Teil anders strukturierte Publikum, vor allem auch durch die enger ausgerichtete Thematik der Dauerausstellung, die erwarteten Besucherzahlen, den Anteil der Besuchergruppen etc., ist für diesen Shop ein völlig neues Konzept zu entwickeln. Auch hier wurden in jüngster Vergangenheit wieder zahlreiche Gespräche mit Interessenten und Shopbetreibern geführt, die zum Ergebnis hatten, daß eine ökonomische Form zur Führung dieser Verkaufsstelle in Leipzig am ehesten unter Einbeziehung von möglichst vielen Synergieeffekten mit dem Shop in Bonn oder 
mit anderen Verkaufsstellen im Kulturmerchandising zu erreichen ist. An dieser Stelle bestätigt sich, daß unbedingt in jedem Einzelfall eine detaillierte Zielsetzung, Analyse und Wirtschaftlichkeitsberechnung notwendig ist, um einerseits dem Besucherinteresse entgegenzukommen und andererseits keinen wirtschaftlichen Schiffbruch zu erleiden.

Für eine wirtschaftliche Führung einer Verkaufsstelle in einem Museum sind bei erster Betrachtung folgende Faktoren von Bedeutung:

- Zahl der antizipierten - oder tatsächlich ermittelten - Museumsbesucher

- Zahl der Käufer im Museumsshop

- Gesamtumsatz

- Umsatz pro Museumsbesucher

- Struktur und finanzieller Einsatz des Warenangebotes

- Anteil der eigenen und zugekauften Produkte

- Umsätze in den einzelnen Produktkategorien

- Personalkosten

- allgemeine Betriebskosten

- Nettogewinn

Beim Vergleich der zur Verfügung stehenden, wenn auch sorgsam gehüteten Umsatzdaten, sollte das Ziel einer Kultureinrichtung in Deutschland sein, ca. zehn Prozent der Besucher zu einem Kauf im Shop zu veranlassen. Bei herausragenden Einzelevents und Ausstellungen könnte dieser Anteil auf 20 bis 25 Prozent zu steigern sein. Internationale Museen mit hohem touristischem Anteil erreichen diese Werte und liegen häufig noch darüber. Wenn auch bei einem erhöhten Anteil der Museumsbesucher als Käufer im Shop der Transaktionswert auf dem relativ hohen Niveau bleibt, der bei geringerem Anteil von Museumsbesuchern im Shop zu konstatieren ist, dürfte dem wirtschaftlichen Erfolg zumindest von dieser Seite her nichts im Wege stehen. 


\section{Eva HofFMEISTER}

\section{Museumsläden in Nordrhein-Westfalen}

\section{Praktische Organisation und Marketingkennzahlen}

Unter der Überschrift "Museumsläden in Nordrhein-Westfalen Praktische Organisation und Marketingkennzahlen " möchte ich den tatsächlichen Stand der Dinge in Sachen rheinischer und westfälischer Museumsläden schildern, so wie ich ihn während einer Serie von Interviews im Frühjahr des Jahres 1997 feststellen konnte. ${ }^{*}$ Die Fragen, die ich den Leiterinnen und Leitern der $\mathrm{Mu}$ seen damals gestellt habe, bezogen sich unter anderem auf Einrichtungs- und Ausstattungsmodalitäten, Entscheidungswege, Bestellwesen, Präsentation der Sortimente und Dekoration sowie die Umsatz- und Besuchsstatistik der Geschäftsjahre 1995 und 1996.

$\mathrm{Zu}$ Beginn gehe ich auf die wichtigsten Stichworte des theoretischen Hintergrundes der Befragung ein. Anschließend werde ich die Befragungsbedingungen vorstellen und danach aufzeigen, in welcher Form das absatzpolitische Instrumentarium des Handelsbetriebes in nordrhein-westfälischen Museen eingesetzt wird sowie schließlich zwei der insgesamt vier ermittelten Kennzahlen in einem Museumsvergleich vorstellen. Am Ende steht - als ein Ergebnis der Studie und meiner eigenen Erfahrung mit der Einrichtung und dem Betrieb von Verkaufsstellen in Museen - die Kurzcharakteristik eines 'guten Museumsladens. 


\section{Theoretischer Hintergrund}

Den Ausführungen zu Zielhierarchie und Handelsmarketing möchte ich zwei Annahmen als gegeben voranstellen:

1. Museen betreiben Marketing.

2. Museumsläden sind Einzelhandelsunternehmen.

\section{Zielhierarchie:}

\section{Unternehmensziele - Handlungsziele - Marketingziele}

Marketingziele einer gewinnorientierten Unternehmung stehen in einer Hierarchie unterschiedlicher Zielebenen. Diese Hierarchie kann als Pyramide dargestellt werden. Die Zahl und der Konkretisierungsgrad der Ziele nimmt von der Spitze zur Basis dieser Pyramide hin zu.

An der Spitze der Pyramide stehen drei übergeordnete Zielebenen: Oberste Priorität im Bestreben, einen Unternehmenserfolg zu erreichen, hat der Unternehmenszweck, der ausdrückt, welche Art von Leistung die Unternehmung erbringen soll, zum Beispiel: „Wir helfen Unternehmen bei der Bewältigung ihrer Informations- und Kommunikationsprobleme."

Der Unternehmenszweck wird gefolgt von der Unternehmensidentität, auch als »Corporate Identity« bezeichnet. Sie soll sich im Verhalten, der Kommunikation und dem Erscheinungsbild der Unternehmung zeigen und sich in der Öffentlichkeit als ihr kennzeichnendes Image ausdrücken. Sie manifestiert sich in vier Bereichen: Corporate Design, Corporate Behaviour, Corporate Communication sowie Unternehmenskultur.

Auf der letzten der drei übergeordneten Zielebenen werden die Unternehmensgrundsätze formuliert, die Leitlinien, mit denen das Unternehmen seine Standpunkte zu wichtigen Fragestellungen beschreibt, z. B.: "Zur Herstellung unserer Produkte verwenden wir ausschließlich nachwachsende Rohstoffe.«Die Grundsätze beziehen sich mit stetig ändernder Priorität auf Kunden, Mitarbeiter, Umwelt sowie Gewinn und Wachstum.

Aus den Ebenen der übergeordneten Ziele ergeben sich die Ebenen der konkreten Handlungsziele: Sie werden angeführt von den Unternehmenszielen. In ihnen legt die Unternehmung die 
Zustände fest, die durch unternehmerische Maßnahmen erreicht werden sollen. Sie können in Gruppen eingeteilt werden:

- Marktstellungsziele (z. B. Höhe des Marktanteils, Umsatzhöhe)

- Rentabilitätsziele (z. B. Höhe des Gewinns oder der Umsatzrentabilität)

- finanzielle Ziele (z.B. Grad der Kreditwürdigkeit oder der Selbstfinanzierung)

- soziale Ziele (z.B. Arbeitszufriedenheit, soziale Integration der Mitarbeiter)

- Prestigeziele (z.B. Unabhängigkeit, Möglichkeit der politischen Einflußnahme)

Aus den bis hier definierten Vorgaben, die miteinander vereinbar sein müssen und bei veränderten Handlungsbedingungen neu aufeinander abzustimmen sind, werden als nächstes die genauen Teilziele für die verschiedenen Aufgabenbereiche der Unternehmung (unter anderem Beschaffung, Marketing, Finanzen) erarbeitet. Mit ihrer Umsetzung wird die Realisierung der Unternehmensziele möglich.

Hängt der Unternehmenserfolg von der Beeinflussung der Absatzmärkte ab, so ist Absatzmarketing (neben Beschaffungsmarketing, Personal- und Finanzmarketing) der Aufgabenbereich der Unternehmung, dem in der Formulierung der Marketingziele besondere Bedeutung zukommt. Mit den Marketingzielen werden auf der untersten Zielebene die Zustände festgelegt, die auf dem Absatzmarkt erreicht werden sollen.

Auch Marketingziele, die sehr viel präziser sind als die vorgenannten Oberziele der Unternehmung, werden in Gruppen eingeteilt. Man unterscheidet ökonomische und psychographische Marketingziele.

Ökonomische Marketingziele, die in engem Zusammenhang zu den generellen Unternehmenszielen stehen, sind z. B. die Höhe des Deckungsbeitrags und des Marktanteils, die Höhe des Gewinns oder der Umsatzrentabilität.

Psychographische Marketingziele beziehen sich auf mentale Prozesse der Konsumenten und betreffen z. B. die Höhe des Bekanntheitsgrades oder die Beeinflussung der Imagebildung und der Kaufabsicht der potentiellen Kunden.

Marketingziele müssen hinsichtlich Inhalt, Ausmaß, Zielgruppen- und Zeitbezug vollständig formuliert sein, sie müssen dem 
anstehenden Bewertungsproblem entsprechen, und sie müssen eine vertikale und horizontale Koordinierung der verschiedenen Zieldimensionen erlauben. Es ist Aufgabe des Managements, die verschiedenen Beziehungen der Ziele untereinander zu erkennen. Die Beziehungen können ergänzender, neutraler oder auch entgegengesetzter Art sein, man spricht von Komplementarität, Neutralität und Konflikt. Weiterhin hat das Management die Aufgabe, die Ziele in ein Zielsystem einzuordnen.

Aus der Festlegung der Zieldimension und ihrer Operationalisierung ergibt sich schließlich die Entwicklung des Marketingplans. Er verbindet Marketingkonzept und Marketingstrategie sowie die Analyse der Zielgruppen und der Ausgangssituation. Im Marketingplan wird der Einsatz des absatzpolitischen Instrumentariums - Marketing-Mix aus Produkt- und Sortimentpolitik, Distributionspolitik, Preis- und Konditionenpolitik sowie Kommunikationspolitik - festgelegt. Der Marketingplan ist ein wesentliches Mittel zur Koordinierung und Lenkung der Marketinghandlungen.

\section{Zielhierarchie im Museumsbereich}

Um die verschiedenen mit der Einrichtung von Museumsläden verbundenen Erwartungen untersuchen zu können, ist es hilfreich, diese Zielhierarchie auf den Museumsbereich zu übertragen: Das Museumsmanagement schreibt in einem ersten Schritt für alle Belange der Einrichtung (Sammlung, Restaurierung / Pflege, Forschung und Vermittlung) die übergeordneten Ziele fest (1. Zweck, 2. Identität, 3. Grundsätze). Alle drei Zielebenen sind abhängig von kulturpolitischem Auftrag, finanzieller Ausstattung oder wissenschaftlichem Anspruch des Museums. Mit der Festlegung dieser Ziele nehmen die Beteiligten - Museumsleitung, Rechtsträger, Sponsor - die Weichenstellung für die Außenwirkung des Museums vor und bestimmen das Qualitätsniveau der Einrichtung. Mit einem Museumsladen können dann z. B. die folgenden Handlungsziele, genauer: `Museums`-Ziele verbunden sein.

\section{Vermittlungsziele}

- Vertiefung der Museumsinhalte durch den Verkauf einschlägiger Literatur

- Vertiefung der Museumsinhalte durch den Verkauf weiterführender, selbst entwickelter Produkte (CD-ROM, Gesellschaftsspiel etc.) 


\section{Imageziele}

- Profilierung des Museums als besucherorientierte Einrichtung (professionell ausgestattete und organisierte Dienstleistungszonen)

- Verbreitung der Marke "Museum« durch den Verkauf von Merchandising-Sortiment

- Einbindung des Museums in das öffentliche Leben durch Kooperationen mit ortsansässigen Produzenten (Kunstgewerbe etc.) und Übernahme ihrer Ware in das Verkaufssortiment

- Einbindung des Museums in seine >Nachbarschaft durch das Angebot von Warengruppen, die auch für Nicht-Museumsbesucher attraktiv sind

\section{Rentabilitätsziele}

- Erzielung von Einnahmen zur Unterstützung des Ankaufsoder Unterhaltsetats

- Erzielung von Einnahmen zur Produktion neuer Verkaufsartikel

\section{Marktforschung}

- durch direkte Kontakte zwischen Verkaufspersonal und Besuchern

Entsprechend diesen Handlungszielen müssen die Museen die Rolle der Museumsläden im Gesamtkonzept "Museums-Marketing « definieren. Bezogen auf die Vermittlungstätigkeit berücksichtigt Marketing für Museen die Bereiche Leistungspolitik (Art, Qualität, Kontinuität der Leistung, Besucherservice), Distributionspolitik (Öffnungszeiten, Erreichbarkeit, "Museum in der Schule«, Absatzmittler), Preispolitik (Eintrittspreise, Ermäßigungen, >Abonnenten-System, Zahlungsbedingungen) und Kommunikationspolitik (Öffentlichkeitsarbeit, persönliche Kontakte, Werbung). Da Verkaufsstellen in Museen zum einen Informationsmedien vertreiben, mit deren Hilfe Ausstellungsinhalte nachvollziehbar gemacht oder vertieft werden können, sind sie im LeistungsMix des Museums besonders zu berücksichtigen. Da zum anderen zumindest ein theoretischer Zusammenhang besteht zwischen der Höhe des Museumseintrittspreises und dem Ausgabeverhalten der Besucher im Museumsladen, ist dieser im Preis- oder Kontrahierungs-Mix zumindest theoretisch $\mathrm{zu}$ berücksichtigen. Und weil zum dritten die Verkaufsstellen von Museumsbesuchern als Teil 
der kulturellen Einrichtung wahrgenommen werden, sind Verkaufsstellen schließlich im Kommunikations-Mix des Museums ebenfalls besonders zu berücksichtigen.

Über die Festlegung von Rentabilitätszielen wirken Verkaufsstellen zusätzlich als Profit-Center der Museen, also als Unternehmensbereich mit Verantwortung für betriebswirtschaftlichen Erfolg und gesondertem Erfolgsausweis. Eine relativ hohe Entscheidungsfreiheit sowie eine auf das Profit-Center bezogene Erfolgsermittlung sind organisatorische Voraussetzung für das Erreichen der Rentabilitätsziele.

\section{Die absatzpolitischen Instrumente des Handelsbetriebes}

Marketing - eine ursprünglich rein absatzmarktorientierte Unternehmenspolitik - findet seit den 8oer Jahren in unterschiedlicher Ausprägung auch in nicht gewinnorientierten Unternehmen Anwendung: Museen bedienen sich - vor allem in Ausübung ihrer Vermittlungsfunktion - der Erkenntnisse des Dienstleistungs-, Nonprofit- oder Kulturmarketing. Verkaufsstellen in Museen sind jedoch Einzelhandelsunternehmen. Mit ihrer Eröffnung ist der quasi unumgängliche Vorgang der Einnahmenerzielung verbunden. Dies bedeutet, daß die Betreiber - wollen sie den Laden erfolgreich führen - Grundsätze des Handelsmarketings anwenden müssen.

Wie im Konsumgüter- und Dienstleistungsmarketing, so leiten sich auch im Handelsmarketing die Marketingziele aus den übergeordneten und den Unternehmenszielen ab. Die Marketingziele sind Basis des Marketingplans, in dem wiederum der MarketingMix definiert wird. Dieser beschreibt die qualitative, quantitative und zeitliche Kombination der Marketinginstrumente.

In Erweiterung der allgemeinen Marketinginstrumente werden unter absatzpolitischen Instrumenten diejenigen speziellen Handlungsmöglichkeiten in der Geschäftspolitik des Handelsbetriebes verstanden, die das Kaufverhalten der aktuellen und zukünftigen Kunden beeinflussen: Gestaltung des Verkaufsraums, Sortiment, Preise und Konditionen, Werbung, Personal. Da der Standort des Museums den Standort des Museumsladens vorgibt - es werden in der Regel (noch) keine »Dependancen« z. B. in Innenstadtlagen eröffnet -, vernachlässige ich an dieser Stelle das Instrument Standortpolitik. 


\section{Verkaufsraumgestaltung}

Ladengestaltung im weiteren Sinn umfaßt die Bereiche Fassade, Schaufenster, Verkaufsraum, Regalflächenoptimierung und Kassenorganisation. Die spezielle Gestaltung des Verkaufsraums wird durch mehrere Faktoren bestimmt:

\section{Laden-Layout}

- Raumaufteilung in verschiedene Funktionszonen (Warenflächen, Kundenflächen, Theken- oder Kassenflächen)

- Raumanordnung innerhalb der Funktionszonen (Regale, Warenträger, Gänge)

\section{Space Utilisation}

- qualitative Raumzuteilung (Zuordnung von Artikeln zu bestimmten Regalplätzen und -flächen)

- quantitative Raumzuteilung (Aufteilung der Regalflächen)

\section{Interior Design}

- Raumeinrichtung durch die Gestaltung der Raumelemente (Möblierung, Boden- und Treppengestaltung etc.)

\section{Atmospherical surroundings}

- Raumumfeldgestaltung (Atmosphäre, Musik etc.)

Ziele der Verkaufsraumgestaltung sind unter anderem die Erhöhung von Umsatz, Deckungsbeitrag, Verweildauer und Einkaufsstättentreue.

Bei der Planung der Inneneinrichtung sind als Handlungsmöglichkeiten möglichst flexible Veränderungen von Lichtverhältnissen, Warenpräsentation und Dekoration vorzusehen.

Ein Kontrollmittel für die Qualität der Verkaufsraumgestaltung ist die Kennzahl Flächenleistung als Relation von Verkaufsfläche in Quadratmetern zum Jahresumsatz.

\section{Sortimentpolitik (Leistungspolitik)}

Als Sortiment wird die Summe aller Absatzobjekte (Sachgüter, Dienstleistungen oder Rechte) bezeichnet, die ein Anbieter im Laufe einer Saison seinen Abnehmern anbieten will.

Sortimente lassen sich durch die Anzahl der alternativ angebotenen Artikelgruppen (Sortimenttiefe), die Anzahl der additiv angebotenen Artikel (Sortimentbreite) sowie die Artikelschwerpunk- 
te (Sortimentrahmen) beschreiben. Wegen der ständigen Anpassung an Marktveränderungen - Wechsel von Bezugsquellen, Änderung von Trends und Kundenwünschen oder der räumlichen Möglichkeiten - unterliegen Sortimente notwendigem Wandel.

Ziele der Sortimentpolitik sind - Gewinnorientierung vorausgesetzt - zum einen die Minimierung der Kosten durch Senkung von z. B. Einstandspreisen und Handlungskosten als auch die Maximierung der Erlöse durch Erhöhung von z.B. Artikelumsatz und Zahl der Kunden.

Das Verkaufsstellenmanagement kann sich folgender sortimentpolitischer Handlungsmöglichkeiten bedienen:

- Einengung des vorhandenen Sortiments durch Streichung einzelner Artikel oder ganzer Artikelgruppen (Sortimentkontraktion),

- Ausweitung des vorhandenen Sortiments durch Hinzunahme einzelner Artikel oder Artikelgruppen (Sortimentexpansion),

- Strukturveränderungen durch Verschieben der Umsatzanteile (Sortimentvariation).

Kontrollmöglichkeiten bezogen auf Umsatz, Deckungsbeitrag und Rentabilität sind:

- Kennzahlenrechnung zur Beurteilung der Umsätze einzelner Artikel,

- Kennzahlenrechnung zur Beurteilung der Umsätze ganzer Warengruppen,

- Analyse der Kundenreaktionen.

\section{Preispolitik}

Als Preis wird diejenige Menge an Geld bezeichnet, die der Käufer für eine bestimmte Menge eines Gutes an den Verkäufer übertragen soll.

Preispolitische Maßnahmen führen zu Veränderungen von Forderungen gegenüber Kunden. Sie können zum einen quantitativer Natur sein (Höhe des Preises), zum anderen zeitlicher (Zeitpunkt der Geldübertragung) oder qualitativer Natur (zulässige Zahlungsmittel).

Das Preisniveau bestimmt sich zum einen nach seiner Fixierung, also nach: 
- Anzahl und Anwendung der Kalkulationssätze (Handelsspannen),

- dem Verhältnis der Preise zu denen der Mitanbieter (Hochoder Niedrigpreispolitik),

- der Häufigkeit, mit der Preise in einem bestimmten Zeitabschnitt geändert werden,

sowie zum anderen nach der Darstellung des Preises durch:

- Bevorzugung einzelner Ziffern (entweder 5 und 9 oder gleiche Ziffern),

- Anzeigen auch anderer Preise (z.B. frühere oder die anderer Anbieter),

- starke Hervorhebung (z. B. große Preisschilder, Symbole).

Im Zentrum des Zielsystems der Preispolitik steht die Gewinnmaximierung. Sie kann begleitet werden von verschiedenen innenoder außengerichteten, lang- oder kurzfristigen Zielen wie der Erhöhung der Liquidität oder dem Abverkauf veralteter Produkte.

Preispolitische Handlungsmöglichkeiten im Rahmen der gesetzlichen Vorschriften sind einerseits die Sonderangebotspolitik bezogen auf ein vorübergehendes Angebot einzelner Waren - und andererseits Verkäufe unter Einstandspreis. Die Entscheidung für eine bestimmte Handlungsmöglichkeit setzt die Möglichkeit ihrer Beurteilung aus Sicht der potentiellen Kunden voraus. Eine solche Beurteilung kann sich auf empirische Daten - Preistests, Konkurrenzforschung, Kundenbefragung - oder auf theoretische Erklärungsansätze stützen.

\section{Werbeplanung}

Die Kommunikationspolitik einer Unternehmung setzt sich zusammen aus Öffentlichkeitsarbeit (Public Relations), Verkaufsförderung, Präsentationspolitik, persönlichem Verkauf, den verschiedenen Formen der nonverbalen Kommunikation (Corporate Design) und Werbung. Als für Handelsbetriebe relevantes Mittel der Kommunikationspolitik möchte ich im folgenden die Werbeplanung vorstellen.

Werbung ist die bewußte, gezielte und kostenverursachende Gestaltung der nichtpersönlichen Kommunikation zwischen der Handelsunternehmung und den aktuellen und potentiellen Kunden unter Einschaltung bestimmter Werbeträger. Werbeträger 
sind Personen oder Sachen, die zur Streuung der Werbemittel eingesetzt werden. Beispiele für Werbemittel und ihre Träger sind Anzeigen in Printmedien, Werbespots in Film, Funk und Fernsehen, besondere Präsentationen im Verkaufsraum, Dekoration im Schaufenster etc.

Ziele der Werbeplanung können ökonomischer Art - z. B. Erhöhung des Umsatzes bestimmter Artikel, Erhöhung des durchschnittlichen Kaufbetrags pro Kunde - oder psychologischer Art sein - z. B. Veranlassung des Besuchs einer Verkaufsstelle, Ausbildung von Kaufabsichten. Sie sind auf die Profilierung der Handelsleistung gerichtet und auf die Schaffung einer wirksamen Nachfrage.

Maßnahmen der Werbeplanung im Rahmen des Werbebudgets sind zum einen die Festlegung von Werbeobjekten nach Inhalt und Art der Darstellung sowie zum anderen die Streuung der Werbung. Auch hier kann zur Vorbereitung der Entscheidungsfindung auf empirische Untersuchungen, Pretests/Posttests, zurückgegriffen werden.

Die Kontrolle der durch Kommunikationspolitik bzw. Werbung erzielten Wirkung ist naturgemäß schwierig, da in der Beeinflussung des Käuferverhaltens alle absatzpolitischen Instrumente sowie Faktoren aus dem Umfeld der Käufer zusammenwirken.

\section{Personalpolitik}

Die kundenorientierte Personalpolitik einer Handelsunternehmung beinhaltet die Bereiche Beschäftigung, Entgelt und Bildung.

Im Rahmen der innerbetrieblichen Beschäftigungspolitik ist $\mathrm{zu}$ entscheiden, wieviele Mitarbeiterinnen und Mitarbeiter mit welchem persönlichen Hintergrund $\mathrm{zu}$ welchen Zeiten wo eingesetzt werden sollen.

Die Entgeltpolitik legt die Höhe der Löhne und Gehälter sowie eventueller Prämienzahlungen fest.

Da die Qualität der Verkaufsgespräche im Zentrum der Beziehungen zwischen Kunden und Verkaufspersonal steht, kommt der innerbetrieblichen Bildungspolitik der Handelsunternehmung besonderes Gewicht zu. Maßnahmen der innerbetrieblichen Bildungspolitik sind Aus- und Weiterbildung sowie Schulung des Personals im Hinblick auf fachliche und persönliche Qualifikation.

Weitere Faktoren, die die Qualität der Beziehungen zwischen Kunden und Personal beeinflussen, sind die generelle Auswahl des Personals, das im Verkauf eingesetzt wird (Kontaktfreudigkeit, 
Auftreten), Anreizlöhne oder andere Arten der Motivation sowie Führungsstil und Kontrolle.

Ziel der Personalpolitik ist es, zu den vorgesehenen Zeiten die jeweils geplante Anzahl von Beschäftigten mit den gewünschten Qualifikationen in den relevanten Tätigkeitsbereichen einsetzen zu können. Dabei ist zu berücksichtigen, daß Mitarbeiter und Mitarbeiterinnen im Handel über die Komponente des Corporate Behaviour das äußere und innere Erscheinungsbild der Unternehmung - Corporate Identity - wesentlich beeinflussen. Dies wiederum hat Auswirkungen auf die Effektivität der übrigen absatzpolitischen Instrumente. Auswahl, Ausbildung und Führung des Verkaufspersonals sind daher ein besonders $\mathrm{zu}$ berücksichtigender Bestandteil des Marketing-Mix.

Zur Kontrolle der quantitativen Personaleinsatzplanung werden vom Management Personalleistungskennzahlen erhoben.

\section{Die Befragung und ihre Ergebnisse}

Zur Beschreibung der Charakteristika von Museumsläden habe ich im Frühjahr 1997 Leiterinnen und Leiter 34 nordrhein-westfälischer Museen zu Organisationsform, Marketing und Wirtschaftsdaten der von ihnen betreuten Verkaufsstellen befragt und ihre Antworten in zwei Stichproben ausgewertet. Die Bildung von zwei Stichproben hatte zum Ziel festzustellen, ob es Unterschiede gibt zwischen den Verkaufsstellen, die vor 1995 eröffneten, und denjenigen, die ihren Betrieb nach November 1995 aufnahmen. Die Stichproben sind wegen der Zurückhaltung mancher Verantwortlicher leider nicht repräsentativ (die Anzahl von Interviews zu Museumsläden in beispielsweise Kunstmuseen entspricht also nicht dem Anteil der Kunstmuseen an allen Museen in NordrheinWestfalen, die Anzahl von Interviews mit externen Pächtern von Museumsläden entspricht nicht der Anzahl der insgesamt in Nordrhein-Westfalen verpachteten Museumsläden etc.).

Da nicht alle befragten Museen bzw. Pächter von Museumsläden, die Fragen zu Organisationsformen und Marketing beantworteten, Angaben zu Umsatzzahlen machen konnten, variieren die Stichprobengrößen in Abhängigkeit von den gestellten Fragen leicht. 
Organisationsform und Marketing

- Stichprobe 1: Umfang: 25 Befragungen; Eröffnung der Verkaufsstellen: 1973 bis April 1995; Betrieb der Verkaufsstellen in 1995

- Stichprobe 2: Umfang: 9 Befragungen; Eröffnung der Verkaufsstellen: November 1995 bis Oktober 1996; Betrieb der Verkaufsstellen in 1996

\section{Wirtschaftsdaten}

- Stichprobe 1: Umfang: 22 Befragungen; Eröffnung der Verkaufsstellen: 1973 bis April 1995; Betrachtung der in 1995 erzielten Umsatzerlöse

- Stichprobe 2: Umfang: 7 Befragungen; Eröffnung der Verkaufsstellen: November 1995 bis Oktober 1996; Betrachtung der in 1996 erzielten Umsatzerlöse

\section{Absatzpolitisches Instrumentarium nordrhein-westfälischer Museumsläden}

Setzen die Verantwortlichen der nordrhein-westfälischen $\mathrm{Mu}$ seumsläden das absatzpolitische Instrumentarium der Handelsbetriebe ein? Einen fachmännischen Umgang mit den entsprechenden Instrumenten konnte ich in den wenigsten Fällen feststellen, statt dessen aber ein hohes Maß an Improvisation und kreativer `Mangelverwaltung`. Welche Ansätze eines Einsatzes sind also erkennbar?

\section{Gestaltung des Verkaufsraums}

Die Mehrheit der Museen hat in ihren Häusern genau eine Verkaufsstelle eingerichtet. Die Verkaufsstellen haben eine durchschnittliche Größe von 81 (Stichprobe 1) bzw. 75 Quadratmetern (Stichprobe 2). Der Zugang zu den Verkaufsstellen ist in Stichprobe $1 \mathrm{zu}$ gleichen Teilen wahlweise und zwangsläufig, in Stichprobe 2 mehrheitlich zwangsläufig. Bis auf den Museumsladen in einem Freilichtmuseum befinden sich alle untersuchten Verkaufsstellen vor den Eingangskassen der Museen. Das Erscheinungsbild der Verkaufsstellen ist zu unterscheiden in Buchhandlung bzw. Laden, Verkaufsbereich, Kiosk und Theke. Die Verkaufsstellen beider Stichproben sind in der Mehrzahl als Läden eingerichtet. Die Dekoration des Sortiments erfolgt in beiden Stichproben fast ausschließlich durch Personal des Museums. In 
Ausstattung, Dekoration und Präsentation des Verkaufssortiments variieren die Verkaufsstellen erheblich.

\section{Sortimentpolitik}

Sortimente in Museumsläden lassen sich unterscheiden in ständig geführte Kernsortimente, vorübergehend angebotene Sondersortimente zu Wechselausstellungen, sowie Zusatz- und Randsortimente. Bestandteile des Kernsortiments sind museumseigenes Sortiment (Kataloge, Kurzführer, Plakate, Postkarten, eigene Videos, CDs oder CD-ROMs), eigenes Merchandising-Sortiment (T-Shirts, Bleistifte, Tassen etc.), Sortimente aus Eigenproduktion (im Museum während Handwerksvorführungen hergestellte Artikel wie Backwaren, Drechselarbeiten, Papier etc.) sowie Bücher. Die Anteile der einzelnen Warengruppen am Gesamtumsatz wurden in den Erfolgsrechnungen der Museen leider nicht erfaßt.

\section{Zahlungsmodalitäten als Bestandteil der Preispolitik}

Wegen der hohen Anschaffungs- und Unterhaltskosten sowie der geringen Kundennachfrage ist in beiden Stichproben Bezahlung mittels Kredit- oder EC-Karte nur in wenigen Verkaufsstellen möglich. Lediglich eine Minderheit der Verkaufsstellen verfügt über ein elektronisches Warenwirtschaftssystem und damit über die Möglichkeit, alle wesentlichen statistischen Daten (Bestellmengen, Lagerbestände, Umschlagshäufigkeit, Einstands- und Verkaufspreise, Deckungsbeiträge, Kennzahlen etc.) jederzeit abrufen zu können.

\section{Werbeplanung}

Für die Mehrheit der Verkaufsstellen beider Stichproben wird kein Werbeplan erstellt. In je fünf Einrichtungen beider Stichproben finden jedoch - mehr oder weniger häufig - Werbemaßnahmen statt. Dies sind z. B. Hinweise auf die Verkaufsstelle im Internet, Anzeigenschaltung, Hinweise in Hausprospekten oder hauseigenen Publikationen.

\section{Personalpolitik}

Im Durchschnitt der untersuchten Verkaufsstellen sind montags bis freitags eineinhalb (Stichprobe 1) bzw. eine (Stichprobe 2) Person beschäftigt. An Wochenenden und Feiertagen sind zwei bzw. ebenfalls eine Person in der Verkaufsstelle tätig. Eine geziel- 
te und regelmäßige Schulung des Verkaufspersonals findet nur in Ausnahmefällen statt.

\section{Kennzahlen und Betriebsvergleich}

Wirken sich diese - teilweise grundverschiedenen - Ausgangsbedingungen auf das Ausgabeverhalten der Besucher und damit auf die Höhe der in einem bestimmten Zeitraum zu erzielenden Verkaufserlöse aus? Theoretisch ja. Aber wie kann dies nachgewiesen werden?

Mit der Erstellung einer auf Museumsläden bezogenen Systematik von Umsatzkennzahlen und Betriebsvergleichen habe ich versucht, Verkaufserlöse in Museumsläden transparenter und damit nachvollziehbarer zu machen. Ich gehe dabei davon aus, daß Museen materiell hinsichtlich ihres Standorts und des angebotenen Verkaufssortiments sowie formell hinsichtlich der Erhebung ihrer Umsatz- und Besuchszahlen vergleichbar sind. Es sei jedoch deutlich darauf hingewiesen, daß es sich wegen der kleinen Stichproben zur Zeit tatsächlich snur um einen Versuch handeln kann. Unabhängig von den Stichprobengrößen hat sich aber die eigentliche Systematik auch in nachfolgenden Berechnungen bewährt. Ziel ist es, in einer zukünftigen Befragung auf breiterer Basis, die praktischen Auswirkungen der verschiedenen Ausgangsbedingungen anhand neu zu berechnender Kennzahlen und Betriebsvergleiche tatsächlich nachzuweisen.

Von den insgesamt vier ermittelten Kennzahlen möchte ich Ihnen zwei vorstellen, nämlich den Umsatz je Museumsbesuch sowie den Umsatz je Stunde Öffnungszeit (und vernachlässige damit die Flächen- und die Personalleistungskennzahl). Die Kennzahlen wurden auf folgendem Weg errechnet:

1. (Jährlicher Umsatz aus Erlösen in Verkaufsstellen) : (Anzahl der Museumsbesuche) = Umsatz je Museumsbesuch (abgekürzt $\mathrm{U} / \mathrm{Mb}$ )

2. (Jährlicher Umsatz aus Erlösen in Verkaufsstellen) : (Gesamtöffnungszeit der Verkaufsstelle in Stunden) = Umsatz je Stunde Öffnungszeit (abgekürzt U/h)

Die Umsätze aus dem Verkauf von Eintrittskarten und Führungsentgelte sind nicht Teil der Umsätze aus Erlösen in Verkaufsstel- 
len. Die Untersuchung ergab die folgenden Spitzenwerte der ermittelten Kennzahlen:

\section{Abbildung 1}

\begin{tabular}{|c|c|c|c|c|c|c|}
\hline \multicolumn{1}{|c|}{ Kennzahl } & \multicolumn{3}{|c|}{ Stichprobe 1 } & \multicolumn{3}{c|}{ Stichprobe 2 } \\
\hline alle Angaben in DM & Rang 1 & Rang 2 & Rang 3 & Rang 1 & Rang 2 & Rang 3 \\
\hline & Spezial-M. & Kunst-M.1 & Freil.-M. & Kunst-M.1 & Kunst-M.2 & Spezial-M. \\
Umsatz je Museumsbesuch & 6,64 & 6,38 & 4,94 & 6,25 & 4,69 & 3,10 \\
\hline Umsatz je Stunde Öffnungszeit & $1.044,00$ & 559,33 & 469,33 & 184,84 & 140,69 & 122,55 \\
\hline
\end{tabular}

Basis: 22 (Stichprobe 1, 1995) bzw. 7 (Stichprobe 2, 1996); Quelle: Hoffmeister 1997

Als Anhaltspunkte seien die niedrigsten Werte der Kennzahlen ebenfalls aufgeführt: Umsatz je Museumsbesuch: 0,23 DM (Stichprobe 1) bzw. 1,07 DM (Stichprobe 2); Umsatz je Stunde Öffnungszeit: 14,47 DM (Stichprobe 1) bzw. 5,97 DM (Stichprobe 2).

Im Verlauf der Jahre 1997 und 1998 hat sich gezeigt, daß in Kunstmuseen sogar ein Umsatz je Museumsbesuch in Höhe von 8 DM (inklusive Katalogverkauf) erzielt werden kann.

Auch unter den angestellten Betriebsvergleichen treffe ich eine Auswahl und stelle an dieser Stelle je zwei Vergleiche bezogen auf Merkmale der Verkaufsstellen und bezogen auf Merkmale der $\mathrm{Mu}$ seen vor.

\section{Vergleich nach Zugangsmöglichkeit}

Die Verkaufsstellen in den untersuchten nordrhein-westfälischen Museen befinden sich in der Regel auf einem den Besuchern vorgegebenen Weg, nämlich vor oder im Bereich der Eingangskasse. Potentiellen Käufern wird dadurch die Möglichkeit geboten, die Verkaufsstelle zu betreten, ohne vorher den Museumseintrittspreis bezahlen zu müssen. Lediglich in Freilichtmuseen ist unter Umständen der Verkaufsbereich auf dem eigentli- 
chen Ausstellungsgelände und nicht im Kassenbereich des Museums plaziert.

Größere Unterschiede zwischen den Einrichtungen ergeben sich bei der Untersuchung der Zwangsläufigkeit des Zugangs zu den Verkaufsstellen. Zwangsläufig ist ein Zugang dann, wenn Besucher auf ihrem Weg zur Eingangskasse oder auf dem Weg zum Ausgang des Museums direkt an einer Verkaufstheke vorbei oder durch eine Verkaufsstelle hindurchgehen müssen (z. B. im Eingangsbereich des Deutschen Museum Bonn, der gleichzeitig $\mathrm{Mu}$ seumsladen ist). Im Gegensatz dazu ist der Zugang fakultativ, wenn Besucher die Wahl haben, den Verkaufsbereich zu betreten oder eben nicht zu betreten (z. B. den durch eine Glasfront räumlich abgeschlossenen Museumsladen im Foyer des Hauses der Geschichte, Bonn). Abbildung 2 gibt die Verteilung der Verkaufsstellen nach ihrer Zugangsmöglichkeit wieder.

Abbildung 2: Verteilung der Verkaufsstellen nach Zugangsmöglichkeit

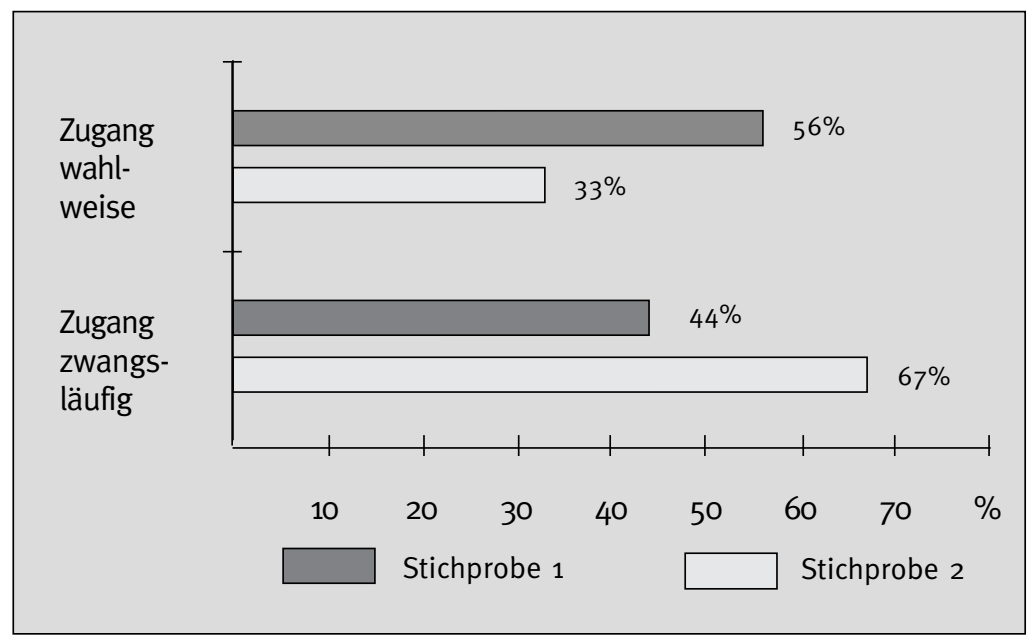

Basis: 25 (Stichprobe 1, 1995) bzw. 9 (Stichprobe 2, 1996); Quelle: Hoffmeister 1997

Abbildung 3 zeigt, wie sich die Zugangsmöglichkeit auf die erzielten Umsatzerlöse ausgewirkt haben könnte. 


\section{Abbildung 3}

\begin{tabular}{|c|c|c|c|}
\hline Stichprobe 1 / Anzahl & $\mathbf{1 1}$ & $\mathbf{1 1}$ & ges. 22 \\
\hline Zugangsmöglichkeit & $\begin{array}{c}\text { wahl- } \\
\text { weise }\end{array}$ & $\begin{array}{c}\text { zwangs- } \\
\text { läufig }\end{array}$ & $\begin{array}{c}\text { Mittelwert } \\
\text { aller } \\
\text { Vk-Stellen }\end{array}$ \\
\hline Umsatz je Museumsbesuch & 3,18 & 1,86 & 2,52 \\
\hline Umsatz je Stunde Öffnungszeit & 280,25 & 70,59 & 175,42 \\
\hline
\end{tabular}

\begin{tabular}{|c|c|c|c|}
\hline Stichprobe 2 / Anzahl & $\mathbf{1}$ & $\mathbf{6}$ & ges. 7 \\
\hline Zugangsmöglichkeit & $\begin{array}{c}\text { wahl- } \\
\text { weise }\end{array}$ & $\begin{array}{c}\text { zwangs- } \\
\text { läufig }\end{array}$ & $\begin{array}{c}\text { Mittelwert } \\
\text { aller } \\
\text { Vk-Stellen }\end{array}$ \\
\hline Umsatz je Museumsbesuch & $\mathrm{xxx}$ & 3,23 & 3,15 \\
\hline Umsatz je Stunde Öffnungszeit & $\mathrm{xxx}$ & 93,53 & 96,22 \\
\hline
\end{tabular}

Alle Angaben in DM; Basis: 22 (Stichprobe 1, 1995) bzw. 7 (Stichprobe 2, 1996); Quelle: Hoffmeister 1997

Stichprobe 1: Entgegen der Erwartung, daß das Betreten einer Verkaufsstelle per se die Kaufbereitschaft des Besuchers erhöht, liegt der $\mathrm{U} / \mathrm{Mb}$ bei wahlweisem Zugang zur Verkaufsstelle deutlich höher als bei zwangsläufigem Zugang. Die zweite Kennzahl steht in umgekehrtem Verhältnis: U / h sinkt um die Hälfte.

Stichprobe 2: Die Kennzahlen des Museums, das als einziges einen wahlweisen Zugang anbietet, sind aus Gründen des Datenschutzes nicht angegeben. Da der U/Mb mit 3,23 DM bei zwangsläufigem Zugang höher liegt als der $\mathrm{U} / \mathrm{Mb}$ bei wahlweisem $\mathrm{Zu}$ gang in Stichprobe 1, könnte in Auswertung der Kennzahlen dieser Stichprobe jedoch die Annahme bestätigt werden: Mit zunehmendem 'Zwang`, die Verkaufsstelle betreten oder passieren zu müssen, steigt der U/Mb.

\section{Vergleich nach Art des Erscheinungsbildes}

Ein wichtiges Instrument des Handelsmarketings ist, wie schon erwähnt, das Ladenlayout. Neben der Schaufenstergestaltung sind 
vor allem die Möglichkeiten der Verkaufsraumgestaltung für Betreiber von Museumsläden von Interesse. Zu unterscheiden sind hier die Gestaltung des eigentlichen Ladens (Ladenarchitektur, Flächenaufteilung, Möblierung, Regalanordnung, Beleuchtung etc.) und die Warenpräsentation (Warenplazierung, Displaygestaltung, Dekoration). Um die Auswirkung der unterschiedlichen Einrichtungsarten auf die Höhe der Umsatzerlöse prüfen zu können, werden die Verkaufsstellen verschiedenen Einrichtungskategorien zugeordnet.

\section{Buchhandlung}

Ladengeschäft, in dem neben Büchern zunehmend auch elektronische Medien verkauft werden, zusätzliches Postkarten- und Plakatsortiment, dabei kein oder nur geringer Anteil an Merchandising- oder Design-Artikeln. Beispiele sind die Buchhandlungen im Wallraf-Richartz Museum, Museum Ludwig, Köln, und Heinz Nixdorf Museums-Forum, Paderborn,

\section{Laden}

Nach Wahrig Deutsches Wörterbuch "Raum (mit Schaufenster), in dem Waren verkauft werden«, gekennzeichnet durch reine oder partielle Selbstbedienung (offene Warenpräsentation in Regalen etc., gleichzeitig Präsentation in Vitrinen, die nur vom Verkaufspersonal zu öffnen sind). Beispiele finden sich im Haus der Geschichte und im Deutschen Museum, beide Bonn.

\section{Verkaufsbereich}

Nach Art der Verkaufsraumgestaltung, Warenpräsentation und Kassenumgebung zwischen Laden und Theke einzuordnen (kein separat abschließbarer Raum, aber doch größere Grundfläche; zum Teil einfache oder zweckentfremdete Möblierung). In dieser Art eingerichtet sind beispielsweise die Verkaufsstellen im Museum für Angewandte Kunst, Köln, und im Leopold-Hoesch-Museum, Düren.

\section{Kiosk}

Nach Wahrig »frei stehendes Verkaufshäuschen oder Stand ...« Ware und meist auch Eintrittskarten werden dem Kunden durch ein Fenster oder eine schalterähnliche Öffnung aus dem Verkaufsraum gereicht. Beispiele für eine solche Verkaufssituation sind das 
Kassenhaus des Archäologischen Parks Xanten und der Kassenbereich im Glasmuseum Rheinbach.

\section{Theke}

Gr., lat. Behältnis, nach Wahrig ursprünglich »Schanktisch«, also meist längerer Verkaufstisch, eventuell ergänzt um Postkarten-, Plakat- oder Bücherständer, meist gleichzeitig Kassentresen zum Verkauf der Eintrittskarten. In dieser Art eingerichtet sind der Kassenbereich des Rheinischen Landesmuseums Bonn und der des Gustav-Lübcke-Museums in Hamm.

Die Häufigkeitsverteilung unter den befragten Museen ist die folgende:

Abbildung 4: Verteilung der Verkaufsstellen nach Art des Erscheinungsbildes (Mehrfachnennung)

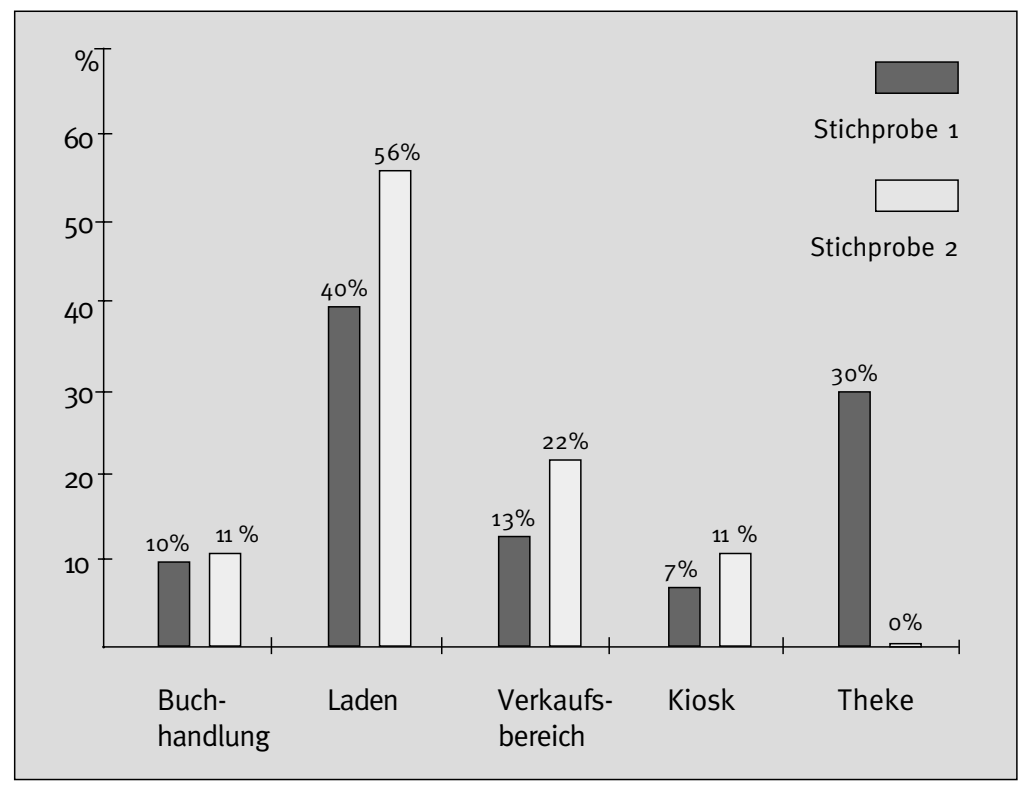

Basis: 25 (Stichprobe 1, 1995) bzw. 9 (Stichprobe 2, 1996); Quelle: Hoffmeister 1997

Abbildung 5 zeigt, wie sich die Art der Einrichtung auf die erzielten Umsatzerlöse ausgewirkt haben könnte. 
Abbildung 5

\begin{tabular}{|c|c|c|c|c|c|}
\hline Stichprobe 1 / Anzahl & $\mathbf{1 0}$ & $\mathbf{5}$ & $\mathbf{2}$ & $\mathbf{5}$ & $\mathbf{g e s .} 22$ \\
\hline Erscheinungsbild & $\begin{array}{c}\text { Buchhandlg. } \\
\text { bzw. Laden }\end{array}$ & $\begin{array}{c}\text { Verkaufs- } \\
\text { bereich }\end{array}$ & Kiosk & Theke & $\begin{array}{c}\text { Mittelwert } \\
\text { aller } \\
\text { Vk-Stellen }\end{array}$ \\
\hline Umsatz je Museumsbesuch & 3,25 & 2,87 & 0,56 & 1,48 & 2,52 \\
\hline Umsatz je Stunde Öffnungszeit & 261,90 & 180,69 & 63,67 & 41,90 & 175,42 \\
\hline
\end{tabular}

\begin{tabular}{|c|c|c|c|}
\hline Stichprobe 2 / Anzahl & $\mathbf{4}$ & $\mathbf{3}$ & ges. $\mathbf{7}$ \\
\hline Erscheinungsbild & $\begin{array}{c}\text { Buchhandlg. } \\
\text { bzw. Laden }\end{array}$ & diverse & $\begin{array}{c}\text { Mittelwert } \\
\text { aller } \\
\text { Vk-Stellen }\end{array}$ \\
\hline Umsatz je Museumsbesuch & 3,62 & 2,52 & 3,15 \\
\hline Umsatz je Stunde Öffnungszeit & 104,18 & 85,61 & 96,22 \\
\hline
\end{tabular}

Alle Angaben in DM; Basis: 22 (Stichprobe 1, 1995) bzw. 7 (Stichprobe 2, 1996); Quelle: Hoffmeister 1997

Stichprobe 1: Der relativ hohe Umsatz in Buchhandlungen bzw. Läden läßt vermuten, daß ein Zusammenhang besteht zwischen Käuferverhalten und Erscheinungsbild der Verkaufsstelle. Zu berücksichtigen ist, daß an Theke und Kiosk in der Regel gleichzeitig der Eintrittskartenverkauf stattfindet.

Stichprobe 2: Auch hier scheint die Kaufbereitschaft der Käufer/Besucher in den als Buchhandlungen bzw. Läden eingerichteten Verkaufsstellen größer zu sein als in den nach anderer Art gestalteten Verkaufsstellen.

\section{Vergleich nach Museumsart}

Die Museen wurden entsprechend ihren Hauptsammelgebieten und -schwerpunkten verschiedenen Museumsarten zugeordnet. Die Einteilung erfolgte nach der in Anlehnung an die UNESCOKlassifikation vorgenommene Gliederung des Instituts für Museumskunde. Nach dieser Einteilung werden Freilichtmuseen auch hier nicht separat dargestellt. An der Umfrage beteiligten sich fünf 
Freilichtmuseen, die ebenso wie ein Firmenmuseum in Stichprobe 1 enthalten sind. Kunst- und Ausstellungshäuser sind der Gruppe der Kunstmuseen zugeordnet.

\section{Abbildung 6: Verteilung nach Museumsart}

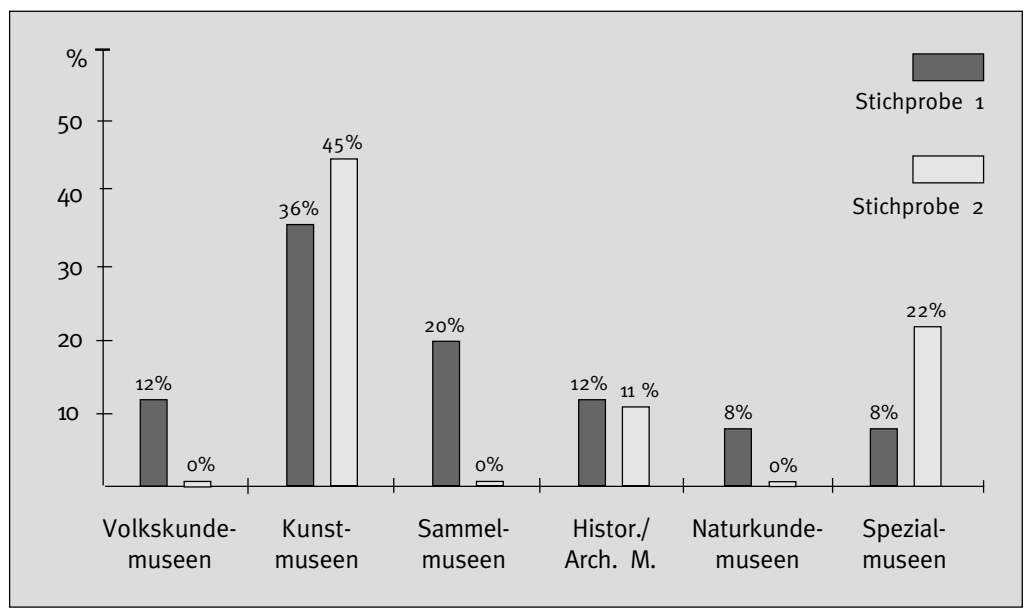

Basis: 25 (Stichprobe 1, 1995) bzw. 9 (Stichprobe 2, 1996); Quelle: Hoffmeister 1997

Abbildung 7 (siehe S. 52) zeigt, wie sich die Museumsart auf die erzielten Umsatzerlöse ausgewirkt haben könnte.

Stichprobe 1: Wegen der außergewöhnlich hohen Standardabweichung in der Gruppe der Spezialmuseen ist der für diese Museen ermittelte Wert des $\mathrm{U} / \mathrm{Mb}$ nicht aussagekräftig. Der Kennzahl $\mathrm{U} / \mathrm{Mb}$ der Kunstmuseen kommt im Verhältnis zum relativierten Wert der kleinen Gruppe der Spezialmuseen größere Bedeutung zu. Erlöse aus Katalog- und Kurzführerverkäufen sind in der Regel leider nicht separat auszuweisen und deshalb auch in der Gruppe Kunstmuseen Bestandteil der errechneten U / Mb.

Stichprobe 2: Die Werte der Kennzahlen der beiden untersuchten Kunstmuseen sind insofern zu relativieren, als beide Verkaufsstellen im letzten Quartal 1996 eröffneten und deshalb der Anteil des Weihnachtsgeschäfts mit höheren Umsätzen größer ist als in den anderen Gruppen. Erlöse aus Katalogverkäufen können ebenfalls nicht separat ausgewiesen werden. 
Abbildung 7

\begin{tabular}{|c|c|c|c|c|c|c|c|c|}
\hline Stichprobe 1 / Anzahl & $\mathbf{3}$ & $\mathbf{7}$ & $\mathbf{4}$ & $\mathbf{4}$ & $\mathbf{2}$ & $\mathbf{2}$ & $\mathbf{g e s .} \mathbf{2 2}$ & davon $\mathbf{4}$ \\
\hline Art der Museen & $\begin{array}{c}\text { Volks- } \\
\text { kunde- } \\
\text { museen }\end{array}$ & $\begin{array}{c}\text { Kunst- } \\
\text { museen }\end{array}$ & $\begin{array}{c}\text { Sammel- } \\
\text { museen }\end{array}$ & $\begin{array}{c}\text { Histor./ } \\
\text { archäol. } \\
\text { Museen }\end{array}$ & $\begin{array}{c}\text { Natur- } \\
\text { kunde- } \\
\text { museen }\end{array}$ & $\begin{array}{c}\text { Spezial- } \\
\text { museen }\end{array}$ & $\begin{array}{c}\text { Mittelwert } \\
\text { aller } \\
\text { Vk-Stellen } \\
\text { Freilicht- } \\
\text { museen }\end{array}$ \\
\hline Umsatz je Museumsbesuch & 2,72 & 3,19 & 2,34 & 1,00 & 0,67 & 5,12 & 2,52 & 2,25 \\
\hline Umsatz je Stunde Öffnungszeit & 247,02 & 169,55 & 54,80 & 120,09 & 86,69 & 529,23 & 175,42 & 204,79 \\
\hline
\end{tabular}

\begin{tabular}{|c|c|c|c|c|}
\hline Stichprobe 2 / Anzahl & $\mathbf{2}$ & $\mathbf{2}$ & $\mathbf{3}$ & ges. 7 \\
\hline Art der Museen & $\begin{array}{c}\text { Kunst- } \\
\text { museen }\end{array}$ & $\begin{array}{c}\text { Technik- } \\
\text { museen }\end{array}$ & diverse & $\begin{array}{c}\text { Mittelwert } \\
\text { aller } \\
\text { Vk-Stellen }\end{array}$ \\
\hline Umsatz je Museumsbesuch & 5,47 & 2,57 & 1,99 & 3,15 \\
\hline Umsatz je Stunde Öffnungszeit & 94,29 & 76,73 & 110,50 & 96,22 \\
\hline
\end{tabular}

Alle Angaben in DM; Basis: 22 (Stichprobe 1, 1995) bzw. 7 (Stichprobe 2, 1996); Quelle: Hoffmeister 1997

\section{Vergleich nach Höhe des normalen Eintrittspreises}

Innerhalb der befragten Museen gibt es die in Abbildung 8 dargestellten Gruppen in Abhängigkeit von der Höhe des normalen Eintrittspreises.

Abbildung 9 zeigt, wie sich die Höhe des Eintrittspreises auf die erzielten Umsatzerlöse ausgewirkt haben könnte. Die Kennzahlen der Museen, die die einzigen in ihrer Kategorie sind, werden aus Gründen des Datenschutzes wieder nicht angegeben.

Stichprobe 1: Die Gruppe der Museen mit einem Eintrittspreis in Höhe von 15 bis 20 DM ist nicht aufgeführt, da nur ein Museum einen Eintrittspreis in dieser Höhe vorsieht. Mit steigender Höhe des Eintrittspreises scheinen auch die Werte des U/Mb zuzunehmen. Eine Ausnahme bildet die Gruppe der Museen mit einem Eintrittspreis in Höhe von 7 bis 8 DM. Das Ergebnis wird bei Aufschlüsselung der deutlich größten Gruppe (Eintrittspreis 5 bis 6 DM) allerdings relativiert. 
Abbildung 8: Verteilung der Museen nach Höhe des normalen Eintrittspreises (für Erwachsene)

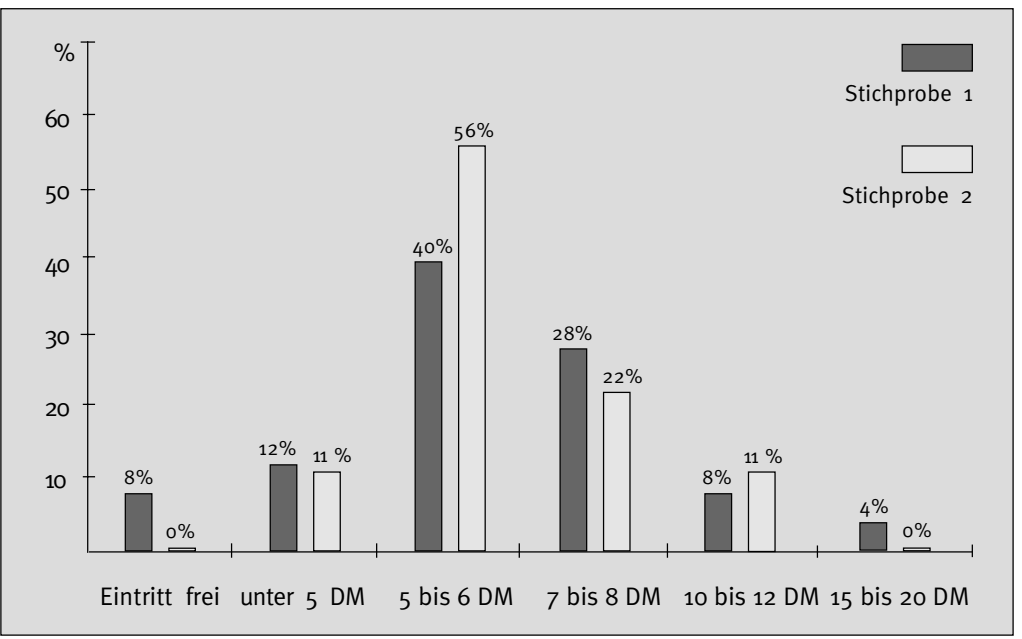

Alle Angaben in DM; Basis: 25 (Stichprobe 1, 1995) bzw. 9 (Stichprobe 2, 1996); Quelle: Hoffmeister 1997

\section{Abbildung 9}

\begin{tabular}{|c|c|c|c|c|c|c|c|c|}
\hline Stichprobe 1 / Anzahl & 2 & 3 & 10 & 4 & 2 & ges. 22 & davon 7 & bzw. 3 \\
\hline \multirow[t]{2}{*}{ Museumseintrittspreis } & \multirow{2}{*}{$\begin{array}{c}\text { Eintritt } \\
\text { frei }\end{array}$} & \multirow{2}{*}{$\begin{array}{l}\text { unter } \\
5 \mathrm{DM}\end{array}$} & \multirow{2}{*}{$\begin{array}{l}5 \text { bis } \\
6 \mathrm{DM}\end{array}$} & \multirow{2}{*}{$\begin{array}{l}7 \text { bis } \\
8 \mathrm{DM}\end{array}$} & \multirow{2}{*}{$\begin{array}{l}10 \mathrm{bis} \\
12 \mathrm{DM}\end{array}$} & \multirow{2}{*}{$\begin{array}{l}\text { Mittelwert } \\
\quad \text { aller } \\
\text { Vk-Stellen }\end{array}$} & \multicolumn{2}{|c|}{5 bis $6 \mathrm{DM}$} \\
\hline & & & & & & & $\begin{array}{l}\text { Kunst- } \\
\text { museen }\end{array}$ & $\begin{array}{l}\text { andere } \\
\text { Museen }\end{array}$ \\
\hline Umsatz je Museumsbesuch & 1,47 & 2,28 & 2,91 & 2,05 & 3,46 & 2,52 & 3,52 & 1,48 \\
\hline Umsatz je Stunde Öffnungszeit & 192,00 & 25,25 & 148,69 & 195,42 & 546,61 & 175,42 & 180,82 & 73,73 \\
\hline
\end{tabular}

\begin{tabular}{|c|c|c|c|c|c|}
\hline Stichprobe 2 / Anzahl & $\mathbf{1}$ & $\mathbf{3}$ & $\mathbf{2}$ & $\mathbf{1}$ & ges. $\mathbf{7}$ \\
\hline Museumseintrittspreis & $\begin{array}{c}\text { unter } \\
5 \mathrm{DM}\end{array}$ & $\begin{array}{c}5 \mathrm{bis} \\
6 \mathrm{DM}\end{array}$ & $\begin{array}{c}7 \mathrm{bis} \\
8 \mathrm{DM}\end{array}$ & $\begin{array}{c}10 \mathrm{bis} \\
12 \mathrm{DM}\end{array}$ & $\begin{array}{c}\text { Mittelwert } \\
\text { aller } \\
\text { Vk-Stellen }\end{array}$ \\
\hline Umsatz je Museumsbesuch & $\mathrm{xxx}$ & 4,53 & 2,79 & $\mathrm{xxx}$ & 3,15 \\
\hline Umsatz je Stunde Öffnungszeit & $\mathrm{xxx}$ & 100,31 & 90,91 & $\mathrm{xxx}$ & 96,22 \\
\hline
\end{tabular}

Basis: 22 (Stichprobe 1, 1995) bzw. 7 (Stichprobe 2, 1996); Quelle: Hoffmeister 1997 
Stichprobe 2: Die Werte der Kennzahlen scheinen mit steigender Höhe des Eintrittspreises zu sinken. Das Ergebnis wird relativiert durch die nicht angegebenen Daten. Alle Werte der Kennzahlen des Museums mit einem Eintrittspreis unter 5 DM liegen niedriger als die der Museen mit einem Eintrittspreis in Höhe von 5 bis 6 DM.

\section{Der 'gute، Museumsladen}

Was sind nun die Kriterien eines iguten`, also bestmöglich funktionierenden Museumsladens? Der sgute، Museumsladen erfüllt aus Sicht der Museen drei Funktionen. Er ist

- Teil der Leistungspolitik des Museums über das Angebot von Informationsmedien, mit deren Hilfe Ausstellungsinhalte vertieft werden können,

- Teil der Kommunikationspolitik des Museums über eine museumsgerechte Gestaltung des Verkaufsraums, den Aufbau persönlicher Kontakte zwischen Verkaufspersonal und Besuchern/Kunden sowie die Abgabe von werbend wirkendem Material,

- Profit-Center für das Museum über die Erzielung von Einnahmen.

\section{Voraussetzungen hierfür sind}

- das Engagement eines kompetenten Verkaufsstellen-Managements das den Anspruch hat, die Verkaufsstelle als Marketinginstrument des Museums zu führen und dabei gleichzeitig das Potential eines Handelsbetriebs bestmöglich ausschöpfen will,

- eine enge, kooperative Zusammenarbeit zwischen Verkaufsstellen-Management und Museumsleitung,

- die Festlegung genauer Zielvereinbarungen hinsichtlich Leistung, Kommunikation und Rentabilität,

- das Engagement von kompetentem Verkaufspersonal und dessen regelmäßige Schulung,

- der Einsatz eines leistungsfähigen Abrechnungssystems. 
Und noch ein weiteres Kriterium möchte ich anführen: Das Management sguter 'Museumsläden kopiert nicht die Konzepte anderer, sondern entwickelt ein auf die jeweilige besondere Ausgangssituation abgestimmtes eigenes Einrichtungs-, Sortiment- und Dekorationsprofil. Damit erst wird die Verkaufsstelle zu einer für den Besucher und für den Betreiber akzeptablen Bereicherung der Gesamtleistung Museum.

\section{Anmerkung}

* Näheres hierzu ist nachzulesen in der Veröffentlichung "Museumsläden und Marketing«, der große Teile dieses Beitrags entnommen sind (Hoffmeister, Eva (1998): Museumsläden und Marketing, Köln).

\section{Literatur}

\section{Zu Fragen der allgemeinen Marketingtheorie:}

Benkert, Wolfgang (1992): "Marketing und Controlling in öffentlichen Kultureinrichtungen«. In: Handbuch KulturManagement. Loseblattsammlung, D 1.2, Stuttgart.

Benkert, Wolfgang/Lenders, Britta/Vermeulen, Peter (Hg.) (1995): KulturMarketing, Stuttgart.

Diller, Hermann (Hg.) (1994): Vahlens Großes Marketing Lexikon, München.

Hasitschka, Werner (1995): »Kulturmarketing«. In: Bruno Tietz (Hg.) 1995.

Holch, Julian (1995): »Dienstleistungsorientiertes Kulturmarketing«. In: Benkert/Lenders/Vermeulen (Hg.) 1995: 27-56.

Kotler, Philip (1992): Marketing-Management, Stuttgart.

Kotler, Philip/Bliemel, Friedhelm (1995): Marketing-Management, Stuttgart.

Lenders, Britta (1995): „Auf dem Weg vom Marketing zum Kulturmarketing «. In: Benkert/Lenders /Vermeulen (Hg.) 1995: 17-26.

Meffert, Heribert (1998): Marketing, Wiesbaden.

Müller-Hagedorn, Lothar (1993): Kulturmarketing, Hagen.

Müller-Hagedorn, Lothar (1996): Einführung in das Marketing, Darmstadt.

Nieschlag, Robert/Dichtl, Erwin/Hörschgen, Hans (1994): Marketing, Berlin.

Raffée, Hans /Wiedmann, Klaus-Peter (1995): "Nonprofit-Marketing«. In: Tietz (Hg.) 1995. 
Scheuch, Fritz (1989): Marketing, München.

Tietz, Bruno (Hg.) (1995): Handwörterbuch des Marketing, Stuttgart.

\section{Zu Fragen des Handelsmarketings:}

Ausschuß für Begriffsdefinition aus der Handels- und Absatzwirtschaft (1982): Katalog E, Begriffsdefinition, Köln.

Barth, Klaus (1988): Betriebswirtschaftslehre des Handels, Wiesbaden.

Berekoven, Ludwig (1990): Erfolgreiches Einzelhandelsmarketing, München.

Gümbel, Rudolf (1963): Die Sortimentspolitik in den einzelnen Betrieben des Wareneinzelhandels, Köln/Opladen.

Hansen, Ursula (1990): Absatz- und Beschaffungsmarketing des Einzelhandels, Göttingen.

Müller-Hagedorn, Lothar (1993): Handelsmarketing, Stuttgart.

Sommer, Elmar (1997): "Neue Brücken schlagen - Merchandising avanciert zu einem lukrativen Bindeglied zum Endverbraucher«. display 4/97, S. 33-41.

Tietz, Bruno (1985): Der Handelsbetrieb, München.

\section{Zu Fragen des Controllings:}

Benkert, Wolfgang (1992): "Marketing und Controlling in öffentlichen Kultureinrichtungen«. In: Handbuch KulturManagement. Loseblattsammlung, D 1.2., Stuttgart.

Bramsemann, Rainer (1995): Angewandtes Controlling, Münster, Hamburg.

Fäßler, Klaus/Rehkugler, Heinz/Wegenast, Claudius (1991): Lexikon des Controlling, Landsberg/Lech.

\section{Zu Fragen des Steuerrechts}

Koch, Karl/Scholtz, Rolf-Detlev (1996): Abgabenordnung Kommentar, Köln.

Strachwitz, Rupert Graf (1996): »Rechts-, Organisations- und Trägerschaftsformen für Museen und Ausstellungsbetriebe«. In: Zimmer, (Hg.) 1996: S. 133-154.

\section{Zu Museumsläden}

Ambrose, Timothy (1993): Managing New Museums, Edinburgh.

Butler, Paul (1993): »The way ahead for museum retailing«. Museum Development 5/93, S. 21-24.

Compania Media (Hg.) (1999): Der Museumsshop. Positionen - Strategien - Sortimente. Ein Praxisführer, Bielefeld.

Institut für Museumskunde (1996): "Eintrittspreise von Museen und Ausgabeverhalten der Museumsbesucher«. Materialien aus dem Institut für Museumskunde, Heft 46. 
Maiwald, Christine (1997): „Wirtschaften statt Haushalten«. Museumsblatt, Mitteilungen aus dem Museumswesen Baden-Württembergs $22 / 97$, S. 16-21.

Museum Store Association (1996): The New Store Work Book.

\section{Zu Besucherservice}

Grönroos, Christian (1990): Service Management and Marketing, Lexington/Mass., Toronto.

Günter, Bernd (1997): "Museum und Publikum: Wieviel und welche Form der Besucherorientierung benötigen Museen heute?«. In: Landschaftsverband Rheinland (Hg.) 1997.

Landschaftsverband Rheinland (Hg.) (1997): Das besucherorientierte Museum, Köln.

\section{Sonstige}

Blumentritt, Ulrich (1984): Modell zur ökonomischen Führung von Museen, Pulheim.

Waidacher, Friedrich (1996): Handbuch der allgemeinen Museologie, Wien.

Zimmer, Annette (Hg.) (1996): Das Museum als Nonprofit Organisation, Frankfurt/Main. 

BrigitTe VoswinkeL

\section{Erfolgreiche Museumsshops}

Anmerkungen zu Management und Produktentwicklung

Der Shop - Balance zwischen Kunst und Kommerz

Wer das Lufthansa-Magazin vom September '98 gelesen hat, dem wird der Artikel über die Situation deutscher und internationaler Museen aufgefallen sein. Die Autorin Beatrice Schaechterle weist darauf hin, daß der Museumsbesuch zu den wichtigsten kulturellen Reisemotiven gehört:

Der Erfolg von Museen mißt sich längst nicht mehr nur allein an der Qualität des Dargebotenen. Studien belegen, daß ohne inszeniertes Drumherum Besucherzahlen stagnieren oder zurückgehen. Verwöhnt von Action-Kino, Marketingevents und Erlebnisreisen sucht der $\mathrm{Mu}$ seumskunde auch in den heiligen Hallen nach Sinnesabenteuern.

Dazu gehört selbstverständlich auch ein attraktiver Museumsshop. Speziell bei Kunstmuseen findet man hierzulande - im Gegensatz $\mathrm{zu}$ anderen Ländern, in denen ein solcher Shop fast zwangsläufig zum Museum gehört - noch häufig Hemmungen, Kunst und Kommerz zu vermischen. Konservative Kritiker fürchten, daß die klassischen Aufgaben der Museen - Sammeln, Bewahren, Erforschen, Ausstellen - gefährdet sind und daß die Qualität der Ausstellungen abhängig gemacht werden könnte von der Verkaufsmöglichkeit der Replikate, wie jüngst in einem ZEIT-Artikel geäußert. Diese Vorwürfe kann man z.B. leicht mit Hinweis auf den Louvre ent- 
kräften: dort wurden 1994 vier Millionen Shopartikel verkauft. Hat das den künstlerischen Wert seiner Ausstellungen gemindert? Hier im von der Heydt-Museum konnten seit Eröffnung des Shops Anfang 1990 einige hochwertige Bilder angekauft werden, für die sonst - bedingt durch die prekäre Finanzsituation der Stadt - keine Mittel zur Verfügung gestanden hätten. Durch den Shop werden Beteiligungen an den Druckkosten von Katalogen bestritten, sowie Ausstellungen, Vorträge oder so profane Dinge wie Beleuchtungskörper mitfinanziert.

Einige zeitgenössische Künstler, wie z.B. Keith Haring, veranstalteten keine Ausstellungen ohne angeschlossenen Museumsshop und wiesen dadurch auf die Bedeutung der neuen Warenklasse zwischen Kunst und Kommerz hin, die der Wuppertaler Professor für Ästhetik, Bazon Brock »Theoretische Objekte» nennt:

Seit alters hat man sich der Leistung solcher Objekte im Gebrauch von Souvenirs, Weihegeschenken und Amuletten versichert. Schon die Reisenden des antiken Massentourismus erwarben Souvenirs, um ihre Erinnerungen an Erlebnisse von Kulturerzeugnissen jederzeit aktivieren zu können. Mit dem deutschen Wort "Andenken" wird die Leistung dieser typischen Theoretischen Objekte prägnant gekennzeichnet. Die Souvenirs bieten der Erinnerung ein Zuhause.

Wer die Entwicklung der Museumsshops mit hochgezogenen Augenbrauen beäugt, sei an seinen kulturkritischen Verdacht erinnert, daß man originäre Kunstwerke der Up-to-date Künstler kaum noch von Shopangeboten unterscheiden könne. Künstlerwerke werden selbst zu theoretischen Objekten und zu Souvenirs des Kunstbetriebs.

Ein weiterer Vorwand gegen einen Shop gerade im eigenen $\mathrm{Mu}$ seum ist die Platzfrage. Man benötigt relativ wenig Verkaufsfläche. Nach unseren Erfahrungen gibt es selten Häuser, die dieses Minimum nicht erfüllen. Für einen Shop sprechen u. a. folgende Argumente:

- Durch die ständig wachsende Freizeit der Menschen können neue Besucherstrukturen aufgebaut werden, die ohne Shops den Museen verloren gehen.

- Ein attraktiv gestalteter Shop kann die Schwellenangst vor dem Museum abbauen und so die Besucherzahl erhöhen - schließlich scheint Shopping ein Grundbedürfnis der meisten Men- 


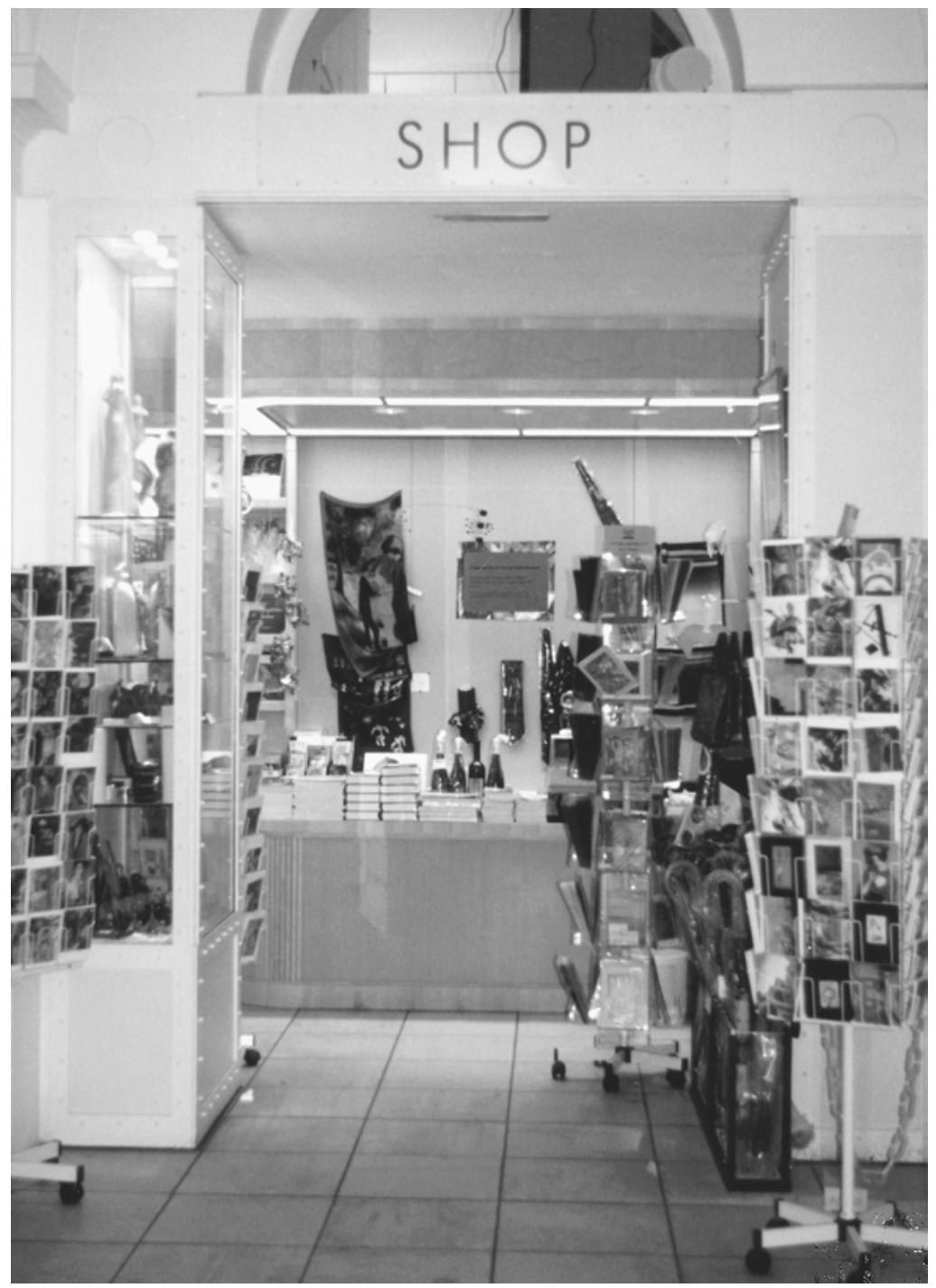

schen zu sein. Warum sollte man ihnen ausgerechnet im $\mathrm{Mu}$ seum diese Möglichkeit verwehren?

- Shopbesucher identifizieren sich mit den im Museum gekauften Artikeln, die wiederum den Beschenkten Kunstverstand suggerieren und somit zur kostenlosen Werbung für das Museum beitragen. Der Käufer hat einen persönlichen Kontakt zu 
der gekauften Ware - ein wesentlicher Vorteil gegenüber dem anonymen Versandhandel.

\section{Die Shopgründung: Grundsatzentscheidungen zum Konzept}

Wenn Sie sich entschlossen haben, einen Shop zu gründen, sollten im Vorfeld die grundsätzlichen Fragen geklärt sein:

\section{Rechtsform}

Vor der Gründung sollten, oder besser noch: müssen Sie verbindliche Gespräche mit den Institutionen führen, mit denen Sie es bestimmt zu tun bekommen, z. B. mit dem Finanzamt. Es gibt eine Vielzahl von möglichen Rechtsformen, ich möchte hier nur zwei erwähnen.

Erfahrungen zeigen, daß bei deutlich weniger als 100.ooo Besuchern der Shop als Zweckbetrieb des Museums über einen gemeinnützigen Verein geführt werden kann und damit die Gewinne ohne Gewerbe-, Umsatz- und Körperschaftsteuer an das Museum zurückfließen. Über diese Besucherzahl hinaus bietet sich die Form der $\mathrm{GmbH}$ an, aber diese Details müssen Sie mit Ihren Beratern klären.

Als Grundkapital für den Ersteinkauf der Waren benötigen Sie etwa 30.000 bis 50.000 DM, die vom Shopträger zur Verfügung gestellt werden müssen. Die Ladeneinrichtung ist in diesem Preis nicht enthalten, da sie meist schon vorhanden ist.

\section{Wohin mit dem Shop?}

Wenn der Shop seinen Auftrag für das Museum erfüllen soll, gehört er zwingend in den Eingangsbereich, er sollte erreichbar sein, bevor man Eintritt zahlt. So kann auch die Laufkundschaft erfaßt werden.

Der Shop im Wuppertaler Von der Heydt-Museum hat ca. $3 \mathrm{om}^{2}$ Verkaufsfläche, doch der Umsatz kann sich sehen lassen. Auch hierbei muß man natürlich die Besucherzahlen berücksichtigen. Ein kleiner, gemütlicher Shop reizt mehr zum Kauf an als ein großer und aufwendig gestalteter. 


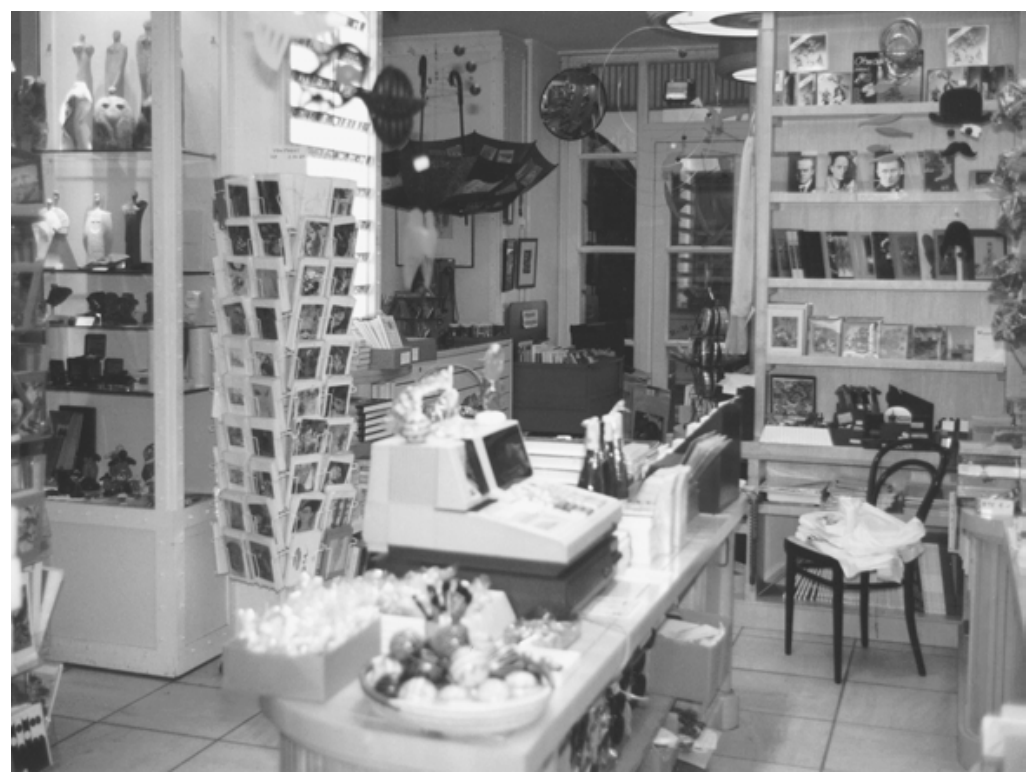

\section{Personal}

Wird der Shop als Zweckverband des Museums über einen gemeinnützigen Verein geführt, dürfen keine Personalkosten aus dem Erlös bezahlt werden. Hieraus ergibt sich zwingend der Einsatz von ehrenamtlichen Kräften. Als Beispiel sei noch einmal das Von der Heydt-Museum genannt: Das Management besteht aus drei Damen: Frau Sunkel und ich sind verantwortlich für Einkauf, Preisgestaltung, Marketing und Organisation, während Frau Reinhardt Kasse und Buchhaltung verwaltet. Wegen der damit verbundenen regelmäßigen Präsenz wird sie aus dem Etat des Museumsvereins bezahlt. Die Verkaufsmannschaft setzt sich aus ca. 50 Damen und wenigen Herren zusammen, fast alle - nach einem Aufruf des Vorstands - von Beginn an dabei. Mit dieser Mannschaft ist es bisher immer gelungen, den Shop während der Museumsöffnungszeiten zu besetzen, auch an Sonn- und Feiertagen sowie in der Urlaubszeit. Die Anwesenheitsregelung erfolgt auf vollkommen freiwilliger Basis, man kann sich in die im Shop ausliegenden Monatspläne eintragen. Unser Dankeschön für die freiwillige Mitarbeit besteht aus einem jährlichen Kaffetrinken zur Adventszeit mit kleinen Geschenken sowie zwei Ausflügen im Jahr, auch das organisiert vom Shopmanagement. 
Wenn als Rechtsform die $\mathrm{GmbH}$ gewählt wurde, sollte man auch soviel freiwillige Helfer wie möglich einsetzen, da der Faktor Personalkosten stets problematisch ist. Bei einem meiner letzten Vorträge wurde ich gerade bei dem Punkt "freiwillige Mitarbeiter" heftig angegriffen: Eine solche Geschäftsführung trage nicht zur Reduzierung der hohen Arbeitslosenzahlen bei. Hierzu nur soviel: Der Zweck eines Shops ist es, Geld für das Museum zu verdienen, nicht die sozialen Probleme der Städte zu lösen. Selbst die deutlich umsatzstärkere Industrie und der Handel können die immensen Personalkosten und vor allem Personalnebenkosten nicht mehr tragen und reduzieren dementsprechend. Außerdem sind in der Regel freiwillige Helfer mit sehr viel mehr Freude dabei, und das wird von den Kunden honoriert. Erst kürzlich ging eine Meldung durch die Presse, wie sehr der Handel durch unfreundliches Personal behindert wird. Unsere Kunden versichern uns immer wieder, wie gern sie zum Einkaufen kommen, da sie so freundlich bedient werden.

Als positives Beispiel kann auch das Naturhistorische Museum in New York angeführt werden, das ebenso viele freiwillige wie fest angestellte Mitarbeiter hat, nämlich je 600 (1994)! Nicht zu unterschätzen dabei ist auch folgender Aspekt: Die überwiegende Zahl der Helfer sind Rentner und Alleinstehende, die dadurch wieder eine Aufgabe für sich gefunden haben und aus der Isolation herauskommen, wobei sie noch das Bewußtsein haben können, einer guten Sache zu dienen.

\section{Einrichtung}

Ein typischer Fehler ist der Wunsch, aus dem Laden ein Museum im Museum zu machen oder eine superelegante Galerie einzurichten. Bei der Einrichtung sollten die verkaufspsychologischen Erkenntnisse des Handels berücksichtigt werden: offene Bücherregale, niedrige Theke im Verkaufsbereich zum Stapeln der Ware, einige wenige geschlossene Glasvitrinen für teure Artikel, umsatzstarke Displays und im unteren Bereich möglichst viel Platz zur Zwischenlagerung.

\section{Kasse}

Ich glaube kaum, daß heute ein modernes Geschäft ohne computergestütztes Warenerfassungssystem auskommen kann. Die Computerkasse gestattet eine ständige Lagerkontrolle und damit eine notwendige Verkaufsstatistik. Voraussetzung dafür ist aller- 
dings die ständige Pflege des Artikelbestandes in der Kasse. Bei unserem Verkaufspersonal wurde das System nach anfänglichen Ängsten voll angenommen, nachdem man erkannt hatte, daß die Abrechnung über Artikelnummern sehr viel einfacher ist, als alle Preise im Kopf zu haben.

\section{Die Zahlen aus der Praxis:}

\section{Beispiele aus dem Von der Heydt-Museum}

\section{Umsatz, Gewinnstatistik, Lagerhaltung}

In den USA gilt als Richtwert ein Jahresumsatz des Shops von 4,50 DM je Museumsbesucher. Für die Richtigkeit unserer Einkaufspolitik spricht, daß wir diesen Wert von Jahr zu Jahr steigern konnten, 1996 und 1997 um fast 50 Prozent. Der Einzelhandel rechnet mit Umsätzen von ca. $10.000 \mathrm{DM} / \mathrm{m}^{2}$ und Jahr, auch diesen Wert haben wir stets mühelos erreicht. Die Gewinnspannen der einzelnen Artikel sind sehr unterschiedlich, so werden bei uns Kataloge und Poster vom Museum bereitgestellt und bringen für den Shop nur zehn Prozent Gewinn. Bücher werden von einer namhaften Kunstbuchhandlung in Kommission geliefert und erzielen 20 Prozent, aber dafür entfallen die Kapitalkosten. Die restlichen Artikel kalkulieren wir handelsüblich.

Hier eine grafische Darstellung von Umsatz und Gewinn der einzelnen Artikelgruppen. Die Zahlen sind Mittelwerte der letzten Jahre.

Der geringe Umsatz an Büchern fällt deutlich auf, aber er deckt sich mit den Erfahrungen anderer ähnlich gelagerter Museen. Während der Umsatz der vom Museum finanzierten Artikel 33 Prozent beträgt, schrumpft der Gewinn auf nur zwölf Prozent. Dagegen erwirtschaften 32 Prozent der Geschenkartikel 44 Prozent Gewinn! Folglich: Die vielgeschmähten Kleinartikel bringen konstant das meiste Geld.

Der durchschnittliche Warenvorrat beträgt bei uns ca. 100.000 DM, d.h. wir haben dank unserer Einkaufsplanung kaum Lagerhaltung. Die Ware befindet sich im wesentlichen in Verkaufsregalen und Displays im Shop.

Wenig empfehlenswert ist es, in puncto Einkauf den Louvre als Vergleich heranzuziehen: Man war kürzlich gezwungen, eine zusätzliche Lagerhalle anzumieten, ein Kostenfaktor, der absolut 


\section{Abbildung 1}

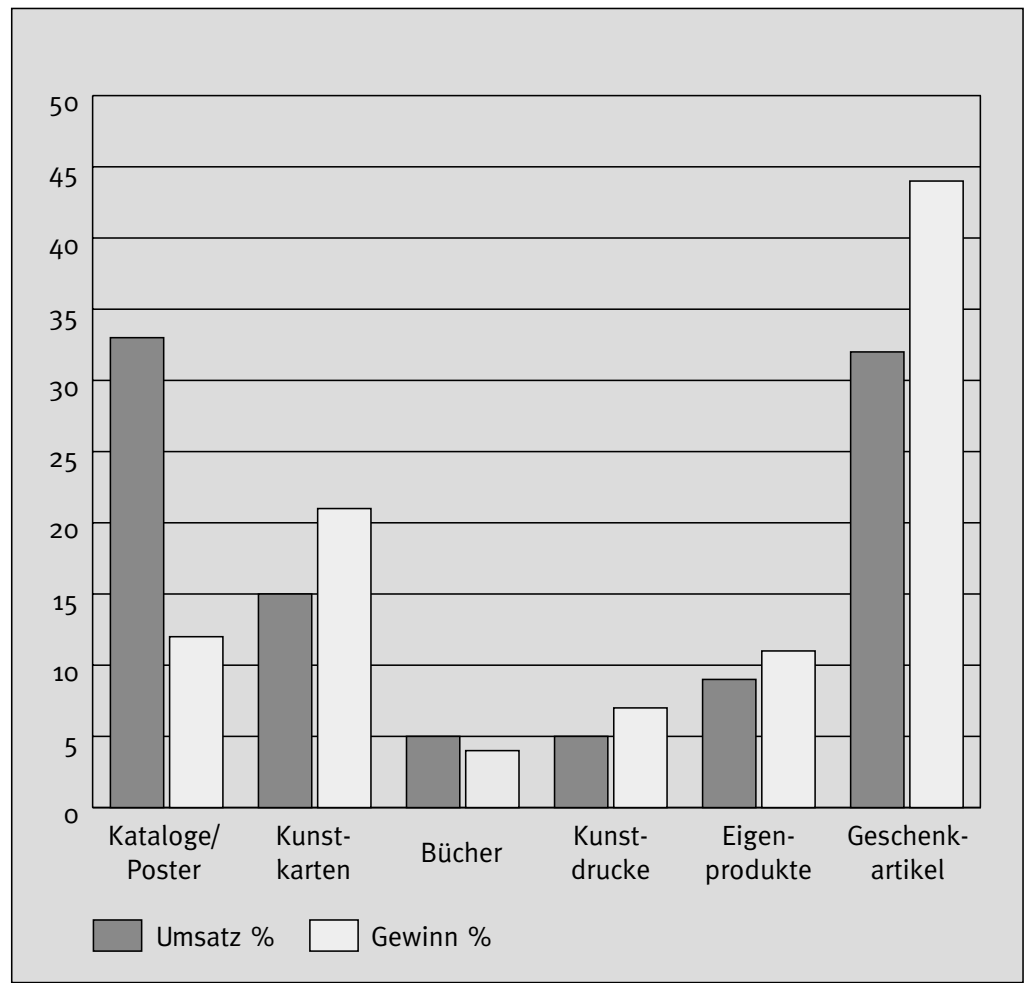

nicht zeitgemäß ist, ich verweise nur auf den Trend der "just in time-Lieferung«, der sich bei Industrie und Handel durchgesetzt hat.

\section{Der Warenkorb:}

Handelsware und museumsbezogene Produktlinien

Das Warenangebot muß selbstverständlich museumsbezogen und auf die Besucherstruktur und -wünsche abgestimmt sein. Preiswerte Waren sollten qualitativ ansprechend sein und hochwertige sind vorsichtig zu disponieren, wobei beim Einkauf unbedingt darauf zu achten ist, nicht nur nach dem eigenen Geschmack zu ordern. Sie sollten für jeden Kunden und jedes Alter entsprechende Artikel bereithalten. Wie beim Handel sollte ein gut sortiertes Impulskaufsortiment an der Kasse gestapelt werden. 
Den größeren Teil der Waren kaufen wir auf Messen ein und geben die Adressen der Händler von shoprelevanten neuen Artikeln an interessierte Shops weiter und tauschen Erfahrungen aus. Aus Zeitgründen verzichten wir bewußt auf Vertreterbesuche.

Trotz des geringen Gewinnanteils von Büchern, wie wir eben in der Verkaufsstatistik gesehen haben, gehören sie in den Shop. Bücher sind immer äußerst dekorativ und untermauern die wissenschaftliche Kompetenz des Museums. Es ist kleineren Museen zu empfehlen, Bücher in Kommission zu nehmen, um ein möglichst breites Angebot ohne Kapitalbindung bieten zu können.

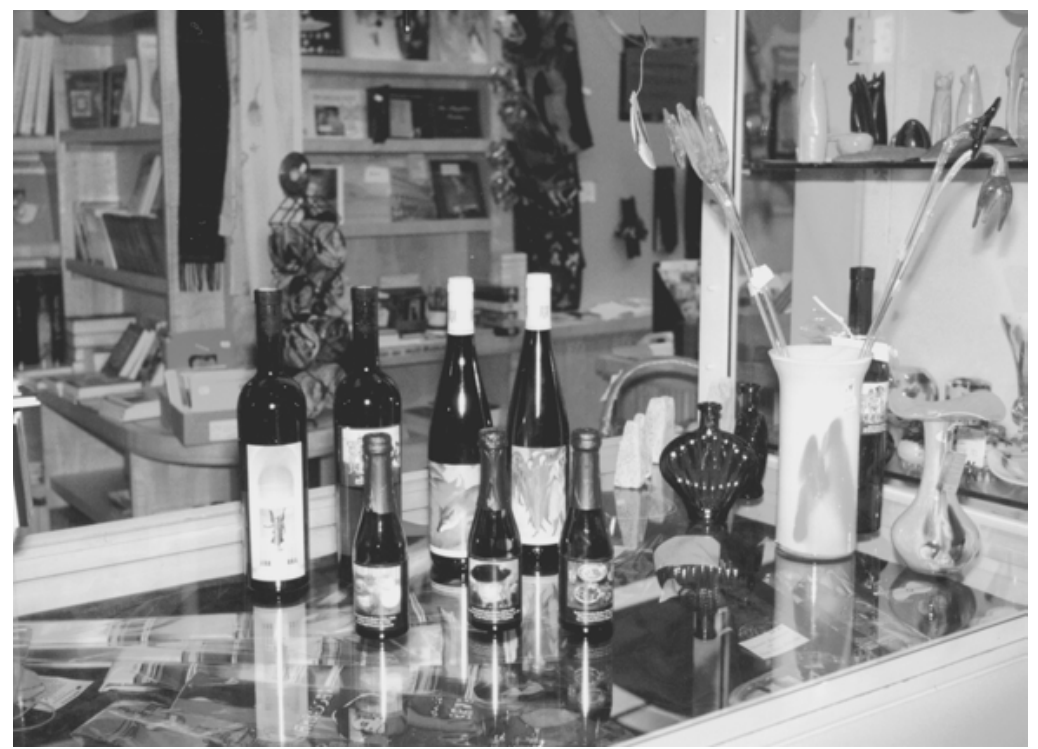

\section{Produktentwicklung}

So lobenswert auch die steigende Zahl von Shops in Museen ist, so birgt sie doch eine große Gefahr in sich: Wohin Sie auch kommen, finden Sie das gleiche Angebot der wenigen großen Anbieter. Um dieser Uniformierung entgegenzuwirken, benötigen Sie ein eigenes Profil. Beschaffen Sie sich also eine möglichst große Anzahl eigener Artikel, die es nur in ihrem Museum gibt. Um gegen Großserienfertigung preislich bestehen zu können, ist eine solche Eigenproduktion natürlich nur sinnvoll, wenn entsprechende Stückzahlen hergestellt werden können. Die Museums Shop Organisa- 
tion (MSO) hat ein Modell entwickelt, bei dem alle teilnehmenden Shops Artikel mit Werken aus dem eigenen Haus herstellen können. Renner bei uns sind Wein und Sekt mit hauseigenen Etiketten, natürlich aus einem der besten Weingüter Deutschlands. Immer gut gehen Blankobücher, Kartenboxen, Mousepads, Geschenkpapier, Servietten und Stofftaschen, alle natürlich speziell für unser Museum hergestellt. Eigenproduktionen sind ferner Schals und Krawatten und einiges mehr. Oft läßt sich auch die Industrie überreden, Artikel, die in ihr Verkaufsprogramm fallen, zu sponsern. Wir entwickeln ständig weitere Ideen, die wir auch anderen Shops zur Mitarbeit anbieten.

\section{Die MSO: Starthilfe und Erfahrungsaustausch beim Aufbau eines Shops}

Wir haben immer wieder festgestellt, daß alle Museen bei der Shopgründung vor den gleichen Fragen und Problemen stehen. Damit nicht jeder das Rad neu erfinden muß, haben Frau Sunkel und ich, nach langjähriger Erfahrung beim Aufbau und der Leitung des Shops im Von der Heydt-Museum Wuppertal, die Museums Shop Organisation gegründet, um unsere Erfahrung weiterzugeben und damit eine rationellere und preiswertere Einkaufsbasis zu erreichen. Wir bieten unsere - für Museen kostenlose - Beratung bei allen shoprelevanten Problemen an, wie z. B. der Frage nach verkaufsstarken Artikeln, deren Einkauf, der Möglichkeit zur Profilfindung über Eigenartikel zu vertretbaren Preisen, der optimalen Präsentation und was es noch für kleine Tips und Kniffe gibt, die man im Laufe der Jahre lernt.

Ich hoffe, ich habe ein wenig dazu beigetragen, die Berührungsängste gegenüber der Vermarktung von Kunst abzubauen und dem hochernsten Kulturbetrieb eine Prise "Erlebnismuseum " hinzuzufügen. 


\section{BERND GÜNTER}

\section{Integration von Museumsshops in das Marketingkonzept von Museen}

Museumsshops sind in den vergangenen Jahren zu einem teils akzeptierten, teils umstrittenen, teils angefeindeten Zweig der Aktivitäten von Museen, ihren Trägern und kommerziellen Anbietern geworden. Der Grad der Akzeptanz reicht von Ablehnung wegen drohender Verflachung und Kommerzialisierung einerseits bis hin zur Forcierung von Museumsläden andererseits, um dringend notwendige Einnahmen zu erzielen. Dabei scheint die Akzeptanz unter anderem auch vom Typ des Museums abzuhängen. Während Museumsshops in technisch orientierten und auch in historischen Museen eher auf ein positives Echo stoßen, sind insbesondere die Angebote von Läden in Kunstmuseen durchaus umstritten. Außerdem darf man vermuten, daß die Akzeptanz bei verschiedenen Besucher- und Nutzerschichten unterschiedlich ausfällt. Auf besonders kritische Positionen von Fachwissenschaftlern des jeweiligen Museumstyps und von Fachjournalisten weisen diverse Veröffentlichungen, auch in den Feuilletons, hin. Insgesamt scheinen Funktion, Bedeutung und Wirkungen von Museumsshops bisher wenig untersucht, speziell im deutschsprachigen Raum. Eine pilotartige empirische Untersuchung für ein Freilichtmuseum - jedoch mit interessanten generellen Hinweisen haben Helm/Klar (1997) durchgeführt und publiziert. Hoffmeister (1998) hat den Versuch unternommen, eine breitere Befragung zu realisieren und auszuwerten, deren Ergebnisse auszugsweise in diesem Band vorgestellt werden. 
Museumsshops sind ein Phänomen, das - neben anderen Facetten - besonders auch unter Management- und Marketing-Gesichtspunkten analysiert werden kann und sollte. Zum einen, weil die Angebote von Museumsläden unter Marketing-Aspekten zu würdigen sind. Zum anderen aber auch, weil Existenz und Betrieb eines Shops eine beträchtliche Bedeutung für das Marketing eines Museums und für das Museumsmanagement generell besitzen.

Auch aus den vorstehend genannten Gründen ist in der gebotenen Kürze darauf hinzuweisen, was Marketing im Museumskontext zweckmäßigerweise bedeutet. Marketing ist nicht einfach mit Werbung oder Verkaufsförderung oder Marktanalysen gleichzusetzen. Ein derartig verengtes Verständnis würde an dem vorbeigehen, was »Marketing " im umfassenden Sinne und im englischsprachigen Wortsinne bedeutet: auf Märkten agieren, Angebote machen und dafür bei Zielgruppen Akzeptanz (»Zu-Wendung«) erzielen. Nach Grönroos (1990) bedeutet Marketing, Beziehungen zu Kunden und anderen Parteien zu etablieren, aufrechtzuerhalten und auszubauen - und zwar in einer Weise, die allen beteiligten Parteien Nutzen bringt und ihnen erlaubt, ihre Ziele zu verfolgen.

Museen sind im Rahmen ihrer bekannten Aufgabenbereiche und Zielsetzungen "Sammeln, Forschen, Bewahren, Erhalten, Bilden und Ausstellen« (ICOM Deutschland 1998) auf verschiedenen Märkten tätig. Sie bieten Leistungen an und pflegen Umgang mit Besuchern, Nutzern und anderen Adressaten, bei denen sie Akzeptanz für ihre Leistungen zu erreichen trachten. Dem dienen Marketing-Überlegungen im Rahmen eines Managements, das seine Ziele effektiv erreichen will und mit knappen Ressourcen effizient umzugehen bestrebt ist (Helm/Klar 1997: Kapitel 1 und 2; Kotler/Kotler 1998).

Museen und Museumsläden, die ohne Bemühen um Effektivität und Effizienz betrieben werden, stellen Verschwendung von Ressourcen dar. Es ist von ihnen also professionelles Management und Marketing zu fordern. Das »Lagerkämmerchen mit Kaufgelegenheit« ist definitiv ein Auslaufmodell; eine systematische Marketing-Konzeption darf heute erwartet werden. Dies gilt sowohl für Shops, die von Museen geführt werden, als auch für solche Geschäfte, die in oder im Umfeld eines Museums von Dritten betrieben werden und ihre Sortimente auf das Museum beziehen.

Museumsshops sind dem Wettbewerb ausgesetzt und müssen daher Wettbewerbsvorteile besitzen bzw. suchen, entwickeln und 
ausbauen: Wettbewerbsvorteile gegenüber den Angeboten, die die Zielgruppen des Museums als Alternativen in Erwägung ziehen, wenn die Planung eines Museumsbesuches auch nur in Frage kommt. Museen konkurrieren in diesem Sinne um vier Ressourcen ihrer Zielgruppen: um deren Zeitbudget, deren Finanzbudget, um deren Bereitschaft zur Weiterempfehlung im Rahmen der Mundwerbung und um deren Bereitschaft zum Engagement für das $\mathrm{Mu}-$ seum, etwa in der kulturpolitischen Debatte. Museen wie auch deren Shops benötigen also Wettbewerbsvorteile in der Wahrnehmung und Beurteilung durch die Adressaten; sonst werden ihre Angebote nicht Präferenzen erzeugen.

Museumsshops können zur Vorziehenswürdigkeit, zur Einzigartigkeit, zur Unverwechselbarkeit, also zu Wettbewerbsvorteilen von Museen beitragen - und sei es als "trojanisches Pferd", wenn Besucher vorzugsweise Shop-Angebote wahrnehmen (wollen), dabei aber zum Besuch des Museums veranlaßt werden können. Damit wird deutlich, daß die Verträglichkeit der Museumsinhalte mit dem Auftritt und den Angeboten eines Museumsladens eine zentrale Gestaltungsaufgabe ist, und die Herstellung der Kompatibilität eine Aufgabe des Museumsmanagements ist. Ein interessantes aktuelles Beispiel für Fragen der Kompatibilität zwischen Museum/Ausstellung und Museumsshop stellt der Fall des Südtiroler Archäologiemuseums in Bozen dar. Dort wird in der Ausstellung der ca. 5.000 Jahre alten Mumie die weltweit bekannte Bezeichnung "Ötzi« streng vermieden und stattdessen vom "Mann aus dem Eis" gesprochen. Im Museumsshop wird der Name »Ötzi« mit großer Selbstverständlichkeit benutzt und in die Vermarktung eingebunden.

Marketing im umfassenden, sintegrierten sinne meint "das Management von Wettbewerbsvorteilen" auf Märkten. Dazu ist das planmäßige Gestalten von Kunden- und Kooperationsbeziehungen erforderlich. Das Vehikel zur Erzielung von Wettbewerbsvorteilen (»besser", »kostengünstiger" oder »schneller" aus der Sicht des Kunden) heißt Kundenorientierung bzw. im Kulturbereich: Besucherorientierung, Nutzerorientierung, Publikumsorientierung oder ganz allgemein Adressatenorientierung. Kundenorientierung bedeutet: nicht immer nur die Innensicht, hier: aus dem Kulturbetrieb Museum nach außen zu pflegen, sondern regelmäßig auch die Außensicht zu überprüfen, d.h. die Sicht eines Besuchers oder Nichtbesuchers auf das Museum einzunehmen. Kunden- bzw. Besucherorientierung beinhaltet z.B. »in den Köpfen 
der Kunden/Besucher spazierenzugehen«. Beispiele dafür sind systematische Stärken-Schwächen-Analysen aus der `Außensicht‘, Besucherforschung und Evaluation, Besucher-Feedback durch den Dialog mit dem Publikum. Die Gründe für das Arbeiten an mehr Besucherorientierung nennt im Sinne eines "Werkzeugkastens der Argumentation« Abbildung 1 (vgl. auch Günter 1998a: $51)$.

\section{Abbildung 1}

\section{Warum mehr Besucherorientierung?}

- damit die Ziele der Präsentation erreicht werden und nicht durch »Besuchermißhandlung « und Unzufriedenheit überlagert werden

- damit Besucher nicht vom »Kern der Sache« abgelenkt werden

- damit Besucher »sich ganzheitlich wohlfühlen« und entsprechend urteilen

- damit positive Mund-zu-Mund-Werbung entstehen kann

- damit Rückkopplung und Verbesserungen/Weiterentwicklung erleichtert werden

- damit nicht Flurschaden durch negative Berichterstattung in den Medien angerichtet wird

- damit Museumsmitarbeiter sich bestätigt fühlen können und zufrieden mit ihrer Tätigkeit sind

- damit - sofern angestrebt - Besuchszahlen gesteigert werden können

- damit man vom Besucher Gegenleistungen erhält (siehe Abbildung 2)

- damit mehr finanzielle Mittel der »öffentlichen Hand« akquiriert werden können

- damit Besucher sich für das Museum engagieren, z.B. in kulturpolitischen Diskussionen.

Kundenorientierung umfaßt sechs `Bausteine`: die Denkweise "vom Kunden her«, Kundenanalyse, Kundensegmentierung, kundenfreundliche Behandlung, Kundenzufriedenheit, Kundenbindung. Dies bedeutet im Zusammenhang mit Museumsshops: die Erwartungen bezüglich Shopangeboten zu ermitteln und - soweit 
mit den Museumszielen verträglich - zu erfüllen; zielgruppenspezifische Angebotspakete zu schnüren; Kundenbehandlung und Serviceorientierung zu pflegen, so wie dies in kommerziellen Bereichen üblich ist oder sein sollte; regelmäßig Kundenzufriedenheit abzufragen; Instrumente einzusetzen, die den Wiederkauf / Wiederholungsbesuch erleichtern, also Besucher zu Stammgästen $\mathrm{zu}$ machen. Wenn es gelingt, besucherorientiert zu handeln, darf ein Museum vom Besucher die in Abbildung 2 aufgelisteten Gegenleistungen vielfältiger Art erwarten, die dazu beitragen, die eigenen Ziele zu erreichen (Günter 1998a: 53).

\section{Abbildung 2}

\section{Gegenleistungen von Museumsbesuchern}

1. Zahlung eines Eintrittspreises, ggf. mit Entgelt für Führung

2. Kauf von begleitendem Informationsmaterial/Museumsführer etc.

3. Kauf von Produkten im Museumsshop aus Interesse, aber auch zur Unterstützung des Museums; Umsätze in der Cafeteria

4. Nutzung des Museums bzw. von Museumseinrichtungen für private/berufliche Zwecke (z. B. Veranstaltung von Feierlichkeiten und Betriebsfesten, Nutzung von Bildmaterial für private und berufliche Zwecke usw., Nutzung der Cafeteria)

5. Zufriedenheit/Lob (Motivationswirkung)

6. Anregungen/Verbesserungsvorschläge aus BesucherFeedback

7. Mund-zu-Mund-Werbung

8. Spenden (Sachspenden, z.B. Exponate; finanzielle Spenden)

9. Mitarbeit, ehrenamtlich z.B. im Freundeskreis bzw. Förderverein (finanzielle Unterstützung, administrative Unterstützung, »inhaltliche Unterstützung«, z.B. wissenschaftliche Mitarbeiter)

10. Unterstützung bei der Verfolgung der Museumsziele in der Öffentlichkeit und bei kulturpolitischen Konflikten. 
Kunden (Museumsbesucher und andere) beurteilen stets die gesamte Qualität eines Kaufvorgangs, eines Museumsbesuches, eines Dienstleistungspaketes, alle Teilleistungen und Merkmale des Angebotes (Helm/Klar 1997). Das bedeutet: Es ist gefährlich, zwischen Rand- und Kernleistungen zu unterscheiden und die Beurteilung von Nebenleistungen durch die Besucher zu unterschätzen. Ein Museumsshop ist nicht eine Randerscheinung, sondern ein Schaufenster des Museums, das den Gesamteindruck von einem Museum und das Gesamterlebnis eines Besuchers mitprägt (Helm/Klar 1997: Kapitel 2 und 5).

Erhebt man die Beurteilung von Museumsshops durch Kunden, so werden Defizite sichtbar, die aufzufüllen sind: im Außenauftritt, in der Information, in der Sortimentsgestaltung und -präsentation, in der Beratung etc. (vgl. Helm/Klar 1997: Kapitel 5, aus dem exemplarisch neuralgische Punkte im Angebot, im Auftritt und in der Beurteilung eines Museumsshops hervorgehen).

Museumsshops, ihre Angebote und ihre Leistungserbringung, müssen - soweit möglich - von Museumsleitungen süberwacht werden, um Kompatibilität mit dem Museumscharakter zu gewährleisten, um ein klares Profil (Ziel-Positionierung) und ein einheitliches Image eines Museums sicherzustellen. Wesentliches Instrument dazu ist bei Außenvergabe der Shop-Leitung die Vertragsgestaltung mit Überwachungsklauseln bzw. Vetorechten.

Das schließt nicht aus, daß Museumsshops ein eigenständiges aber mit dem Museumsprofil kompatibles - `Gesicht` und Erscheinungsbild entwickeln. Dieses sollte geprägt sein durch das Angebot von >Besonderheiten`, von unverwechselbarer Attraktivität (z. B. Herausgabe einer eigenen Edition; spezialisierte Literatursortimente, die im Buchhandel so nicht präsent sind; evtl. eigenes Logo usw.; siehe oben: Wettbewerbsvorteile).

Um Wettbewerbsvorteile eines Shops gegebenenfalls auch separat vom Museumsangebot zur Geltung kommen zu lassen, sollte jeder Museumsshop auch unabhängig vom Museumsbesuch zugänglich und erreichbar sein. Der Vorschlag, Shops stets getrennt von der Kassenzone eines Museums zugänglich zu machen, ist zwar im Einzelfall nicht unproblematisch, aber vor dem Hintergrund der oben beschriebenen Marketing-Konzeption wohlbegründet - abgesehen von kleinen Häusern, für die beide Optionen in Frage kommen. 
Museumsshops können verschiedene Formen der Kooperation - intensiver als bisher - nutzen, z.B. mit anderen Kultureinrichtungen, mit der Gastronomie, mit Verkäufern von Büchern, Materialien, Tonträgern oder regionalen Produkten. Dies gilt insbesondere bei Neuplanungen oder im Rahmen von Umbaumaßnahmen, wobei hier auch an verschiedene Varianten der Einbeziehung potentieller Besucher zu denken ist.

Betreiber von Museumsshops können und sollten intensiv von anderen Betrieben lernen - nicht nur von erfolgreichen Museumsshops, sondern im Sinne des Benchmarkings von Unternehmen in anderen, auch kommerziellen Branchen, die eine »best practice«, etwa bei der Erfüllung bestimmter Funktionen des Merchandising, der Shoppräsentation, der Logistik, des Angebots von Sammlerobjekten usw., realisieren.

Museumsshops stellen eine hervorragende Chance zur Kundenbindung dar - für den Shop und für das Museum. So kann es Aufgabe von Museumsshops sein, durch Unterstützung der Mundwerbung zur Besucherbindung beizutragen oder durch Internet-Präsenz auch das Museum als Ganzes zu fördern.

Die Betreiber von Museumsshops können und sollten die Chance ergreifen, das Ausmaß und die Art von Besucherorientierung zu realisieren, die ein Museum ansonsten nicht pflegt (im Einzelfall vielleicht auch: nicht pflegen will). Eine Chance des $\mathrm{Mu}-$ seumsshops besteht ebenfalls darin, Besuchern und Nicht-Besuchern bestimmte Service- und Informationsleistungen anzubieten, die ein Museum nicht an anderer Stelle bereitstellt.

Abschließend sollen die wichtigsten Problemfelder und Ansatzpunkte für eine praktische Marketing-Konzeption (siehe auch z.B. Helm/Klar 1997: Kapitel 2; Kotler/Kotler 1998) in drei Konstellationen aufgezeigt werden. Es sind drei Fälle in der Museumspraxis zu unterscheiden:

\section{Fall 1: Ein Museum betreibt einen Shop in eigener Regie}

Kernprobleme des Marketing sind:

- das Finden von Besonderheiten (Einzigartigkeit, die Wettbewerbsvorteile ermöglicht) im Waren- und Dienstleistungsangebot 
- fehlende Ressourcen, die bereitzustellen wären (Personal, zeitliche Kapazität usw.)

- Know-how des Museums und seiner Mitarbeiter, z.B. beim Einkauf von Waren und im Merchandising

- Kosten

- Professionalität in Verkauf und Beratung, evtl. sogar Versand,

- Service-Aspekte wie etwa Zahlungsmodalitäten, Öffnungszeiten

- Einstellung zum Kunden und zum Marketing generell

Lösungsmöglichkeiten aus Marketing-Sicht bestehen in: kreativinnovativen Überlegungen zu unverwechselbaren Angeboten, Wirtschaftlichkeitsanalysen und Controlling (ggf. mit externer Hilfe), Schulung/Training, Zukauf einzelner Dienstleistungen oder ganzer >Pakete (»Outsourcing«). Eine gewisse Chance liegt zum Beispiel in der möglichen Sonntagsöffnung von Museumsläden und -kiosken.

Fall 2: Ein Museum verpachtet einen Shop an einen externen Händler/Dienstleister bzw. kooperiert mit einem Shopbetreiber im eigenen Haus bzw. auf dem Museumsgelände

Kernprobleme des Marketing sind:

- Auswahl des Partners und Kontrolle der externen Mitarbeiter (insbesondere ihrer Einstellung zur Ausstellung und zum Kunden/Besucher)

- Kompatibilität mit dem Museum und seinen Zielen

- Kontrolle des Sortiments und der Produktqualität

- Vertragsbedingungen

Lösungsmöglichkeiten aus Marketing-Sicht bestehen in: sorgfältiger Auswahl des Partners, gemeinsamer Festlegung unverwechselbarer Angebote, vertraglicher Festlegung von Inhalten und Konditionen, regelmäßiger Abstimmung und Kontrolle der Partner, Überprüfung der Verträglichkeit durch Image- und Einstellungsanalysen bei Besuchern u. a. 
Fall 3: Es wird ein auf das Museum bezogener Shop außerhalb des Museumsgeländes ohne Zutun und ohne Kooperation mit dem Museum betrieben.

Kernprobleme des Marketing aus der Sicht des Museums sind:

- unkoordinierte und unkontrollierte Konkurrenz

- Intransparenz und >Verwechselbarkeit ‘ bei Besuchern, Museumsmitarbeitern und anderen

- Imageprobleme

- dem Museum entgehen Chancen, Abrundungs- und Einnahmemöglichkeiten

- der spätere Aufbau eines eigenen Museumsladens wird erschwert.

Lösungsmöglichkeiten aus Marketing-Sicht bestehen darin, daß ein Museum eben nicht einer unkontrollierbaren Konkurrenz das Feld überläßt, sondern mit einem geeigneten Angebot zum »Rundum-Erlebnis« der Besucher beiträgt; ferner in einer auf das $\mathrm{Mu}$ seum und der Abgrenzung zu Fremdanbietern aufmerksam machenden Information.

Mit diesen Überlegungen und Ausführungen soll verdeutlicht werden, daß Marketing-Konzeptionen von Museen und Shops aufeinander abzustimmen sind, aber unverwechselbare, attraktive, einzigartige Leistungen in den Vordergrund stellen sollten, so daß die Herausforderung für das Museumsmanagement lautet: »Kompatibilität und Besonderheit«.

\section{Literatur}

Günter, Bernd (1998a): »Besucherorientierung - eine Herausforderung für Museen und Ausstellungen«. In: Marita Anna Scher (Hg.): (Umwelt-)Ausstellungen und ihre Wirkung, Oldenburg, S. 51-55.

Günter, Bernd (1998b): »Wozu braucht ein Museum Besucher?« In: Hans Albert Treff (Hg.) mit dem International Council of Museums ICOM: Museen unter Rentabilitätsdruck. München, S. 67-75.

Helm, Sabrina/Klar, Susanne (1997): Besucherforschung und Museumspraxis, München.

Hoffmeister, Eva (1998): Museumsläden und Marketing, Köln. 
ICOM (International Council of Museums) Deutschland (1998): ICOM Kodex der Berufsethik, München.

Kotler, Neil/Kotler, Philip (1998): Museum Strategy and Marketing, San Francisco. 


\section{Steuerpflicht von Museumsshops}

Das Erscheinungsbild von Museumsshops gestaltet sich in der Praxis vielfältig und reicht vom einfachen Verkaufstisch neben der Museumskasse über separate Räumlichkeiten für den Verkauf bis hin zum Shop im Internet. Die Möglichkeiten, in welchen Rechtsformen solche Läden betrieben werden können, sind ebenfalls sehr zahlreich. So können z. B. die Museumsshops von den Museen selber betrieben werden, oder der Förderverein des Museums betreibt den Museumsshop. Denkbar wäre (ist) aber auch, daß ein »fremder Dritter« den Verkauf organisiert. Bei einem solchen fremden Dritten kann es sich um eine $\mathrm{GmbH}$ oder um eine natürliche Person handeln.

Das Steuerrecht muß alle oben genannten Konstellationen entsprechend erfassen - unter Berücksichtigung der jeweiligen Besonderheiten des Einzelfalles, insbesondere der Rechtsform, in welcher das Museum und der Shop betrieben werden.

Damit eine entsprechende steuerrechtlich korrekte Beurteilung der wirtschaftlichen Tätigkeit erfolgen kann, muß zunächst einmal das Museum als Ganzes gesehen werden, um im zweiten Schritt die entsprechenden steuerrechtlichen Schlußfolgerungen zu treffen. 
Handelt es sich z. B. bei dem Museum um einen Regiebetrieb oder einen Eigenbetrieb einer juristischen Person des öffentlichen Rechts, bedarf es für alle weiteren steuerrechtlichen Beurteilungen grundsätzlich der Überprüfung, ob dieses einen Betrieb gewerblicher Art (BgA) darstellt.

Bei einem Museum einer juristischen Person des öffentlichen Rechts, das gegen Entgelt der Allgemeinheit offensteht und dessen Jahresumsatz (Brutto) im Sinne von $\S 1$ Absatz 1 Nr. 1 Umsatzsteuergesetz (UStG) 60.00o DM nachhaltig übersteigt, handelt es sich steuerrechtlich um einen BgA. Es bedarf keiner Gewinnerzielungsabsicht in diesem Zusammenhang.

Belaufen sich die Einnahmen der gesamten Einrichtung, inklusive der des Museumsshops, unter 60.00o DM, unterliegen diese nicht der Umsatz- und den Ertragssteuern. Bei Einnahmen von über 60.00o DM (inklusive der Eintrittsgelder) bedarf es jedoch aus steuerrechtlichen Gründen einer differenzierteren Betrachtungsweise. Das Museum einer juristischen Person des öffentlichen Rechts ist dann insgesamt unbeschränkt körperschaftsteuerpflichtig, gemäß $\S 1$ Abs. 1 Nr. 6 in Verbindung mit $\S 4$ KStG (Körperschaftsteuergesetz).

Der Steuersatz für solche Einrichtungen beträgt zur Zeit nach Abzug eines Freibetrages von 7.500 DM 42 Prozent. Die meisten Museen entrichten jedoch keine Körperschaftsteuer an die Finanzverwaltung, weil die Steuer nur von einem entsprechenden Gewinn (Betriebseinnahmen minus Betriebsausgaben) zu entrichten ist.

Grundsätzlich wird das gesamte Museum, inklusive des Museumsshops, als ein BgA angesehen. Dies hat zur Konsequenz, daß den Mehreinnahmen aus dem Shopbetrieb Mehrausgaben aus dem sonstigen Museumsbereich, z. B. Personalkosten, gegenüberstehen, so daß die Einrichtung insgesamt keinen Gewinn erwirtschaftet.

Die 60.00o DM-Grenze ist für die Museen unerheblich, welche in der Rechtsform einer GmbH geführt werden. Solche Einrichtungen sind grundsätzlich körperschaftsteuerpflichtig. Auch die o.g. Freibetragsgrenze findet hier keine Anwendung. Der thesaurierte Gewinn unterliegt einem Steuersatz von 45 Prozent. Bei Ausschüttung verringert sich dieser auf 30 Prozent, zuzüglich der 
Kapitalertragsteuer. Gegebenenfalls sind entsprechende Gewinne der Gewerbesteuer zu unterwerfen.

\section{Umsatzsteuer}

Grundsätzlich sind alle entgeltlichen Lieferungen und sonstigen Leistungen, welche die Museen (Einnahmen über 60.00o DM im Jahr) im Leistungsaustausch erbringen, umsatzsteuerbar, weil die Gesamtheit aller Betriebe gewerblicher Art im Sinne von § 1 Abs. 1 Nr. 6, § 4 KStG und aller land- und forstwirtschaftlichen Betriebe das Unternehmen der juristischen Person des öffentlichen Rechts darstellen.

Die Museen müssen jedoch keine Umsatzsteuer an die Finanzbehörden abführen, soweit der Umsatz nach $\S 4$ Nr. 20 a UStG steuerbefreit sind.

\section{§ 4 UStG Steuerbefreiung bei Lieferungen, sonstigen Leistun- gen und Eigenverbrauch}

Von den unter $\S 1$ Abs. 1 Nr. 1 bis 3 fallenden Umsätzen sind steuerbefreit:

...20. a) die Umsätze folgender Einrichtungen des Bundes, der Länder, der Gemeinden oder der Gemeindeverbände: Theater, Orchester, Kammermusikensembles, Chöre, Museen, botanische Gärten, zoologische Gärten, Tierparks, Archive, Büchereien sowie Denkmäler der Bau- und Gartenbaukunst. Das gleiche gilt für die Umsätze gleichartiger Einrichtungen anderer Unternehmer, wenn die zuständige Landesbehörde bescheinigt, daß sie die gleichen kulturellen Aufgaben wie die in Satz 1 bezeichneten Einrichtungen erfüllt. Museen im Sinne dieser Vorschrift sind wissenschaftliche Sammlungen und Kunstsammlungen.

Für die Anwendung der Steuerbefreiungsvorschriften ist es grundsätzlich ohne Bedeutung, ob das Museum und somit der Shop in der Form eines Regiebetriebes oder einer $\mathrm{GmbH}$ betrieben wird.

Ist der einzige Gesellschafter einer solchen $\mathrm{GmbH}$ der Bund, das Land, die Gemeinde oder die Gemeindeverbände, bedarf es m.E. auch keiner landesbehördlichen Bescheinigung, weil es sich immer noch um eine Einrichtung der entsprechenden juristischen 
Person des öffentlichen Rechts handelt. Ansonsten ist eine solche Bescheinigung zwingend erforderlich, damit eine entsprechende Steuerbefreiung nach $\S 4 \mathrm{Nr}$. 20 a UStG gewährt werden kann. Liegt eine solche Bescheinigung für das Museum nicht vor, sind die entsprechenden Umsätze steuerbar und steuerpflichtig. Sie unterliegen jedoch dann dem ermäßigten Steuersatz gemäß § 12 Abs. 2 Nr. 7a UStG.

Jedoch nicht alle Umsätze des Museums fallen unter diese Steuerbefreiung. Dies gilt insbesondere für die Umsätze der $\mathrm{Mu}$ seumsshops. Nähere Ausführungen diesbezüglich enthält der Abschnitt 108 Absatz 2 der Umsatzsteuerrichtlinien:

Steuerfrei sind insbesondere die Leistungen der Museen, für die als Entgelte Eintrittsgelder erhoben werden, und zwar insoweit, als es sich um Sonderausstellungen, Führungen und Vorträge handelt. Die Steuerbefreiung erfaßt auch die bei diesen Leistungen üblichen $\mathrm{Ne}$ benleistungen, z. B. den Verkauf von Katalogen und Museumsführern und die Aufbewahrung der Garderobe. Als weitere typische $\mathrm{Mu}$ seumsleistungen sind anzusehen das Dulden der Anfertigung von Reproduktionen, Abgüssen und Nachbildungen sowie die Restaurierung und Pflege von Kunstwerken in Privatbesitz, die von den Museen im Interesse der Erhaltung dieser Werke für die Allgemeinheit vorgenommen werden. Der Verkauf von Kunstpostkarten, Fotografien, Dias, Plakaten, Klischees, Reproduktionen, Abgüssen, Nachbildungen, Farbdrucken und Bildbänden ist nur dann als typische $\mathrm{Mu}$ seumsleistung steuerfrei, wenn

1. es sich um Darstellungen von Objekten des betreffenden Museums handelt,

2. das Museum die genannten Gegenstände selbst herstellt oder herstellen läßt und

3. diese Gegenstände ausschließlich in diesem Museum vertrieben werden.

Der Verkauf von Literatur, die in Beziehung zu der Sammlung des betreffenden Museums steht, ist bei Vorliegen der Voraussetzungen Nr. 2 und 3 ebenfalls steuerfrei. Die Veräußerung von Museumsobjekten sowie von Altmaterial ist dagegen von der Steuerbefreiung nach $\S 4 \mathrm{Nr}$. 20 UStG ausgeschlossen. Es kann jedoch die Steuerbefreiung nach $\$ 4 \mathrm{Nr}$. $28 \mathrm{UStG}$ in Betracht kommen (vgl. Abschnitt 122). 
Museumsshops dürfen z.B. Kunstpostkarten nur dann umsatzsteuerfrei veräußern, wenn alle drei o.g. Voraussetzungen vorliegen. In diesem Zusammenhang verweise ich insbesondere auf die dritte Voraussetzung, in der es heißt, daß diese Gegenstände ausschließlich in diesem Museum vertrieben werden müssen. Dies bedeutet für die Praxis, werden solche Kunstpostkarten auch außerhalb des Museums vertrieben, ist die Veräußerung von Farbdrucken im Museumsshop steuerbar und steuerpflichtig. Die Steuerbefreiung nach $\S 4 \mathrm{Nr}$. 20 a ist somit ausgeschlossen. Die Umsätze im Museumsshop müssen dann entsprechend der Umsatzbesteuerung unterworfen werden.

Die Aufzählung in der Umsatzsteuerrichtlinie ist hinsichtlich des Umfanges der Steuerbefreiung nicht abschließend geregelt. Somit können auch andere nicht in diesem Abschnitt aufgeführte Lieferungen und sonstige Leistung steuerbefreit sein. Es muß sich jedoch um museale Nebenleistung handeln.

Unterliegt ein Umsatz nicht der Steuerbefreiung nach $\S 4 \mathrm{Nr}$. 20 a UStG, bedingt dies nicht automatisch eine Steuerpflicht, denn auch alle anderen Steuerbefreiungsvorschriften des Umsatzsteuerrechtes können hier Anwendung finden, soweit die entsprechenden Voraussetzung vorliegen. Werden z. B. vom Museum lediglich die Räumlichkeiten, in welchen der Shop betrieben wird, vermietet, handelt es sich hierbei grundsätzlich um einen umsatzsteuerfreien Vorgang, gemäß § 4 Nr. 12 UStG. Dem Museum steht es jedoch frei, mit diesem Umsatz zur Steuerpflicht zu optieren (§ 9 UStG).

Es hängt von den Gegebenheiten des Einzelfalles ab, ob eine solche Option für das Museum aus steuerrechtlichen Gründen günstig ist. Mitvermietete Betriebsvorrichtungen wie z.B. Regale sind immer von der Steuerbefreiung ausgenommen.

Liegt ein umsatzsteuerbarer und umsatzsteuerpflichtiger (Befreiungsvorschriften greifen nicht) Vorgang vor, muß überprüft werden, ob der volle oder der ermäßigte Steuersatz hier Anwendung findet. Welche Umsätze dem ermäßigten Steuersatz unterliegen, regelt der § 12 Absatz 2 abschließend. Werden z. B. im $\mathrm{Mu}-$ seumsshop Bücher verkauft, welche nicht bereits nach $\S 4 \mathrm{Nr}$. 20 a UStG steuerbefreit sind, werden diese Umsätze mit dem ermäßigten Steuersatz, gemäß § 12 Absatz 2 Nr. 1 UStG, versteuert.

Im Gegenzug kann die von einem anderen Unternehmer in Rechnung gestellte Umsatzsteuer als Vorsteuer abgezogen werden. Vom Vorsteuerabzug ausgeschlossen ist insbesondere die 
Steuer für Lieferungen und sonstige Leistungen, die der Unternehmer zur Ausführung steuerfreier Umsätze, hier $\S 4 \mathrm{Nr}$. 20 a UStG, verwendet. Hinsichtlich der sonstigen Voraussetzungen und Besonderheiten sei an dieser Stelle nur auf die Ausführungen des $\S 15$ UStG verwiesen, insbesondere des Absatzes 4, betreffend der Aufteilung von Vorsteuerbeträgen.

Handelt es sich bei dem Museum um einen Betrieb gewerblicher Art, findet die Regelungen für Kleinunternehmer nach § 19 UStG keine Anwendung, weil alle Betriebe gewerblicher Art das Unternehmen der juristischen Person des öffentlichen Rechts bilden und somit die entsprechenden Voraussetzungen nicht vorliegen. Alle anderen Museen können die Kleinunternehmerbesteuerung nach § 19 UStG für sich in Anspruch nehmen, so daß diese keine Umsatzsteuer zahlen müssen. Im Gegenzug können keine Vorsteuerbeträge gegenüber der Finanzverwaltung geltend gemacht werden.

Nach dieser Regelung brauchen die Museen keine Umsatzsteuer zu entrichten, wenn die steuerbaren Brutto-Einnahmen einschließlich des Eigenverbrauchs und die darauf entfallenden Steuern aus seiner gesamten unternehmerischen Betätigung im vorangegangenen Kalenderjahr 32.500 DM nicht überstiegen haben und im laufenden Kalenderjahr voraussichtlich 100.00o DM nicht übersteigen werden. Bei der Ermittlung des Gesamtumsatzes bleiben unter anderem die Umsätze außer Ansatz, welche z. B. nach $§ 4 \mathrm{Nr}$. 11 bis 28 UStG steuerbefreit sind (vgl. § 19 Abs. 3 UStG). Somit sind Vermietungsumsätze und die Eintrittsgelder des Museums nicht in diese Berechnung einzubeziehen.

Museen, welche grundsätzlich als Kleinunternehmer anzusehen sind, können jedoch auf diese Regelung verzichten.

Steuerrechtlich, insbesondere ertragsteuerrechtlich, werden $\mathrm{Mu}$ seen, welche von der Finanzverwaltung als gemeinnützige Einrichtung anerkannt sind, differenzierter beurteilt. Voraussetzung für eine Anerkennung durch die Finanzverwaltung ist jedoch, daß eine entsprechende Satzung vorliegt, die dem Gemeinnützigkeitsrecht im Sinne der Abgabenordnung (§ 51 AO folgende) entspricht und daß die tatsächliche Geschäftsführung mit dieser im Einklang steht.

Liegen diese Voraussetzungen vor, gliedert sich das Museum für die steuerrechtliche Betrachtung in vier Teilbereiche auf. Es handelt sich um den ideellen Bereich, die Vermögensverwaltung, den Zweckbetrieb und den wirtschaftlichen Geschäftsbetrieb. 
In diesem Zusammenhang möchte ich insbesondere auf folgende gesetzliche Vorschriften in der AO verweisen, aus welchen sich die entsprechenden Abgrenzungsmerkmale ableiten lassen:

\section{§ 14 AO Wirtschaftlicher Geschäftsbetrieb}

Ein wirtschaftlicher Geschäftsbetrieb ist eine selbständige nachhaltige Tätigkeit, durch die Einnahmen oder andere wirtschaftliche Vorteile erzielt werden und die über den Rahmen einer Vermögensverwaltung hinausgeht. Die Absicht, Gewinne zu erzielen, ist nicht erforderlich. Eine Vermögensverwaltung liegt in der Regel vor, wenn Vermögen genutzt, z.B. Kapitalvermögen verzinslich angelegt oder unbewegliches Vermögen vermietet oder verpachtet wird.

\section{§ 64 AO Steuerpflichtige wirtschaftliche Geschäftsbetriebe}

(1) Schließt das Gesetz die Steuervergünstigung insoweit aus, als ein wirtschaftlicher Geschäftsbetrieb (§ 14) unterhalten wird, so verliert die Körperschaft die Steuervergünstigung für die dem Geschäftsbetrieb zuzuordnenden Besteuerungsgrundlagen (Einkünfte, Umsätze, Vermögen), soweit der wirtschaftliche Geschäftsbetrieb kein Zweckbetrieb (\$ 65 bis 68) ist.

(2) Unterhält die Körperschaft mehrere wirtschaftliche Geschäftsbetriebe, die keine Zweckbetriebe (\$ 65 bis 68) sind, werden diese als ein wirtschaftlicher Geschäftsbetrieb behandelt.

(3) Übersteigen die Einnahmen einschließlich Umsatzsteuer aus wirtschaftlichen Geschäftsbetrieben, die keine Zweckbetriebe sind, insgesamt nicht 60.0oo Deutsche Mark im Jahr, so unterliegen die diesen Geschäftsbetrieben zuzuordnenden Besteuerungsgrundlagen nicht der Körperschaftsteuer und der Gewerbesteuer.

(4) Die Aufteilung einer Körperschaft in mehrere selbständige Körperschaften zum Zweck der mehrfachen Inanspruchnahme der Steuervergünstigung nach Absatz 3 gilt als Mißbrauch von rechtlichen Gestaltungsmöglichkeiten im Sinne des $§ 42$. 


\section{§ 65 AO Zweckbetrieb}

Ein Zweckbetrieb ist gegeben, wenn

1. der wirtschaftliche Geschäftsbetrieb in seiner Gesamtrichtung da$\mathrm{zu}$ dient, die steuerbegünstigten satzungsmäßigen Zwecke der Körperschaft zu verwirklichen,

2. die Zwecke nur durch einen solchen Geschäftsbetrieb erreicht werden können und

3. der wirtschaftliche Geschäftsbetrieb zu nicht begünstigten Betrieben derselben oder ähnlichen Art nicht in größerem Umfang in Wettbewerb tritt, als es bei Erfüllung der steuerbegünstigten Zwecke unvermeidbar ist.

\section{§ 68 AO Einzelne Zweckbetriebe}

Zweckbetriebe sind auch: ...

7. kulturelle Einrichtungen, wie Museen, Theater, und kulturelle Veranstaltungen, wie Konzerte, Kunstausstellungen; dazu gehört nicht der Verkauf von Speisen und Getränken.

Bezogen auf den Museumsshop bedeutet dies, daß dieser nunmehr einen wirtschaftlichen Geschäftsbetrieb darstellt. Zwar handelt es sich bei Museen gemäß $\S 68$ Nr. 7 AO grundsätzlich um einen Zweckbetrieb, jedoch ist der Verkauf von Speisen und Getränken ausgenommen. Es handelt sich bei dieser Ausnahme um keine abschließende Aufzählung, wie sich bereits aus dem Anwendungserlass zur AO ableiten läßt, in dem es heißt:

10. Der Verkauf von Speisen und Getränken und die Werbung bei kulturellen Veranstaltungen gehören nicht zu dem Zweckbetrieb. Diese Tätigkeiten sind gesonderte wirtschaftliche Geschäftsbetriebe. Wird für den Besuch einer kulturellen Veranstaltung mit Bewirtschaftung ein einheitlicher Eintrittspreis bezahlt, so ist dieser - ggf. im Wege der Schätzung - in einen Entgeltsanteil für den Besuch der Veranstaltung und für die Bewirtschaftungsleistung aufzuteilen.

Auch diese Ausführung beinhaltet keine abschließende Aufzählung, wie die Praxis und die hierzu ergangene Rechtsprechung zeigt. 
Bei Museumsshops handelt es sich nicht um Zweckbetriebe nach $\S$ 68 Nr. 7 AO. Eine entsprechende Anwendung § 65 AO scheidet für die Läden ebenfalls aus, weil diese in einem Wettbewerb zu anderen Anbietern stehen und diese Shops für die Erfüllung der steuerbegünstigten Zwecke nicht zwingend erforderlich sind.

Bezogen auf die einzelnen Steuerarten ergeben sich die nachfolgend dargestellten steuerrechtlichen Konsequenzen.

\section{Körperschaft- und Gewerbesteuer}

Bei den Museumsshops handelt es sich um wirtschaftliche Geschäftsbetriebe, welche in vollem Umfange körperschaftsteuerpflichtig sind, gemäß $\S 5$ Abs. 1 Nr. 9 Körperschaftsteuergesetz (KStG).

\section{§ 5 Befreiungen}

(1) Von der Körperschaftsteuer sind befreit...

9. Körperschaften, Personenvereinigungen und Vermögensmassen, die nach der Satzung, dem Stiftungszweck oder der sonstigen Verfassung und nach der tatsächlichen Geschäftsführung ausschließlich und unmittelbar gemeinnützigen, mildtätigen oder kirchlichen Zwekken dienen ( $\$ 51$ bis 68 der Abgabenordnung). Wird ein wirtschaftlicher Geschäftsbetrieb unterhalten, ist die Steuerbefreiung insoweit ausgeschlossen.

Hieraus ergibt sich im Umkehrschluss, daß der ideelle Bereich, die Vermögensverwaltung und der Zweckbetrieb körperschaftsteuerbefreit sind.

Die Überschüsse aus den Museumsshops sind jedoch nur dann körperschaftsteuerpflichtig, wenn die (Brutto-)Einnahmen aller wirtschaftlichen Geschäftsbetriebe des Museums 60.000 DM im Jahre übersteigen. Unterhält das Museum z. B. einen Shop, dessen Jahresumsatz 45.00o DM beträgt, unterliegt dieser nicht der Körperschaftsteuerpflicht. Erhält dieses Museum für durchgeführte Werbemaßnahmen im gleichen Jahr noch 20.000 DM, unterliegen die wirtschaftlichen Geschäftsbetriebe "Laden" und "Werbung" der Körperschaftsteuer, weil die Einnahmen insgesamt 60.00o DM überschreiten. Die Steuerfreiheit des übrigen Bereiches des $\mathrm{Mu}$ - 
seums (ideeller Bereich, Vermögensverwaltung und Zweckbetrieb) bleibt hiervon unberührt.

Nicht die Einnahmen, sondern der erzielte Überschuß bildet die Bemessungsgrundlage für die Körperschaftsteuer. Aus diesem Grund sind von den Einnahmen die entsprechenden Ausgaben abzusetzen.

Weil eine gemeinnützige Einrichtung aus den oben genannten vier Teilbereichen besteht, von dem nur der wirtschaftliche Geschäftsbetrieb der Körperschaftsteuer unterliegt, ist eine entsprechende Zuordnung der Einnahmen und Ausgaben zu dem jeweiligen Bereich zwingend erforderlich. Entfallen die Einnahmen und die Ausgaben nur auf einen der Bereiche, ist die Zuordnung noch relativ unproblematisch.

Schwieriger gestaltet sich die Zuordnung von Einnahmen und Ausgaben, welche mehrere Bereiche der Einrichtung betreffen. Nach einem Beschluß der obersten Finanzbehörden können in Fällen der gemischten Nutzung Aufwendungen mit dem auf den steuerpflichtigen wirtschaftlichen Geschäftsbetrieb entfallenden Anteil bei der Gewinnermittlung berücksichtigt werden. Dies gilt auch für anteilige Abschreibungsbeträge und anteilige Personalkosten.

Der Steuersatz für gemeinnützige Einrichtungen, mit der Ausnahme der gemeinnützigen $\mathrm{GmbH}$, beträgt zur Zeit nach Abzug eines Freibetrages von 7.500 DM 42 Prozent. Für GmbH beträgt der Steuersatz zur Zeit 45 Prozent.

In diesem Zusammenhang bleibt anzumerken, daß ein dauerhafter Verlust im wirtschaftlichen Geschäftsbetrieb grundsätzlich zur Aberkennung der Gemeinnützigkeit der gesamten Einrichtung führt. Dies ergibt sich aus dem allgemeinen Grundsatz des Gemeinnützigkeitsrechts, daß für die steuerbegünstigten Zwecke gebundene Mittel nicht für den Ausgleich von Verlusten aus steuerpflichtigen wirtschaftlichen Geschäftsbetrieben verwendet werden dürfen.

Bei einer Körperschaft, die mehrere steuerpflichtig wirtschaftliche Geschäftsbetriebe unterhält, ist für die Frage, ob gemeinnützigkeitsschädliche Verluste vorliegen, nicht auf das Ergebnis des einzelnen steuerpflichtigen wirtschaftlichen Geschäftsbetriebs, sondern auf das zusammengefaßte Ergebnis aller steuerpflichtigen wirtschaftlichen Geschäftsbetriebe abzustellen. Danach ist die Gemeinnützigkeit einer Körperschaft gefährdet, wenn die 
steuerpflichtigen wirtschaftlichen Geschäftsbetriebe insgesamt Verluste erwirtschaften.

Ein evtl. entstehender Verlust (unter Berücksichtigung von anteiligen Abschreibungsbeträgen) ist gemeinnützigkeitsrechtlich unschädlich, wenn der Verlust nur dadurch entstanden ist, daß ein Wirtschaftsgut aus dem ideellen Bereich lediglich zur besseren Auslastung und mit der Absicht, zusätzliche Mittel für den ideellen Bereich zu beschaffen, teil- oder zeitweise für einen steuerpflichtigen wirtschaftlichen Geschäftsbetrieb genutzt wird. Dies gilt jedoch nur unter folgenden Bedingungen:

- es wurden marktübliche Preise erzielt,

- es wurde kein größeres Wirtschaftsgut angeschafft, als es für die gemeinnützige Tätigkeit notwendig gewesen wäre und

- der wirtschaftliche Geschäftsbetrieb bildet keinen eigenständigen Bereich eines Gebäudes (z.B. Verkaufsräume eines Museumsladens).

Gewinne des wirtschaftlichen Geschäftsbetriebs sind ebenfalls der Gewerbesteuer zu unterwerfen.

Entsprechend der Freibetragsregelung bei der Körperschaftsteuer steht bei der Ermittlung des Gewerbeertrags ein Freibetrag in Höhe von 7.50o DM zu Verfügung. Nur für den darüber hinausgehenden Gewerbeertrag wird der Steuermeßbetrag nach dem Gewerbeertrag mit fünf Prozent ermittelt.

Vermietet ein gemeinnütziges Museum lediglich die Räumlichkeiten, in denen der Shop betrieben werden soll, an einen Förderverein, handelt es sich aus Sicht des Museums um eine reine Vermögensverwaltung, welche nicht der Körperschaftsteuer unterliegt.

Die Führung des Museumsshops begründet beim Förderverein einen wirtschaftlichen Geschäftsbetrieb, wenn die oben genannten Voraussetzungen gegeben sind.

\section{Umsatzsteuer}

Die von der Finanzverwaltung als gemeinnützig anerkannten $\mathrm{Mu}$ seen sind den sonstigen Einrichtungen, welche eine solche Anerkennung nicht haben (siehe oben), umsatzsteuerrechtlich grundsätzlich gleichgestellt. Lediglich sind hier die den begünstigten 
Zwecken dienenden Umsätze nur mit dem ermäßigten Steuersatz zu versteuern. Dies ergibt sich aus $§ 12$ Absatz 2 Nr. 8 a UStG.

\section{§ 12 UStG Steuersatz}

(2) Die Steuer ermäßigt sich auf sieben vom Hundert für die folgenden Umsätze: ...

8. a) die Leistungen der Körperschaften, die ausschließlich und unmittelbar gemeinnützige, mildtätige oder kirchliche Zwecke verfolgen (§§ 51 bis 68 Abgabenordnung). Das gilt nicht für Leistungen, die im Rahmen eines wirtschaftlichen Geschäftsbetriebes ausgeführt werden;

Soweit somit die getätigten Umsätze in dem Bereich der Vermögensverwaltung und in dem Zweckbetrieb nicht steuerbefreit sind, insbesondere gemäß $\S 4$ Nr. 12 und Nr. 20 a UStG, unterliegen sie nur dem ermäßigten Steuersatz.

Bei Museumsshops handelt es sich jedoch um wirtschaftliche Geschäftsbetriebe. Die in diesem Bereich getätigten Umsätze sind grundsätzlich steuerbar. Findet keine Befreiungsvorschrift, insbesondere der $\S 4$ Nr. 20 a UStG keine Anwendung, sind diese Vorgänge steuerpflichtig. Besonderheiten in der Anwendung der Befreiungsvorschriften im wirtschaftlichen Geschäftsbetrieb sieht der Gesetzgeber nicht vor, so daß die Befreiungsvorschriften auch für den Shopbereich uneingeschränkt Anwendung finden. Aus diesem Grund verweise ich auf meine bisherigen Ausführungen zu diesem Bereich, insbesondere der Anwendung des $\S 4$ Nr. 20 a UStG.

Die Höhe des Steuersatzes richtet sich nach § 12 UStG. Grundsätzlich unterliegen die Umsätze im Shopbereich dem vollen Steuersatz, weil es sich aus steuerrechtlicher Sicht um einen wirtschaftlichen Geschäftsbetrieb handelt und somit der ermäßigte Steuersatz nach $\S 12$ Abs. 2 Nr. 8 a UStG keine Anwendung findet. Jedoch können andere Ermäßigungsvorschriften des § 12 Abs. 2 hier Anwendung finden. Der steuerpflichtige Verkauf von Büchern im Museumsshop unterliegt somit nach $\S 12$ Abs. 2 Nr. 1 UStG nur dem ermäßigten Steuersatz.

Sofern die Museen im Rahmen ihrer unternehmerischen Tätigkeit Leistungen von einem anderen Unternehmen bezogen haben, können diese in Rechnung gestellte Umsatzsteuer als Vorsteuer gegenüber dem Finanzamt geltend machen. Vom Vorsteuerabzug 
ausgeschlossen ist die Steuer für Lieferungen und sonstige Leistungen, welche die Museen, inklusive der Museumsläden als wirtschaftliche Geschäftsbetriebe, zur Ausführung steuerfreier Umsätze verwenden. Hinsichtlich der sonstigen Voraussetzungen verweise ich hier auf die Ausführungen des $§ 15$ UStG, insbesondere des Absatzes 2 und 4, betreffend die Aufteilung von Vorsteuerbeträgen.

Für den Abzug der Vorsteuer unmaßgeblich ist, welchen Bereich (ideeller Bereich, Vermögensverwaltung oder wirtschaftlicher Geschäftsbetrieb) diese Rechnung betrifft. Es kommt ausschließlich darauf an, ob die bezogene Leistung oder Lieferung für die Ausführung von steuerfreien oder steuerpflichtigen Umsätzen verwendet wird.

Die Regelung für Kleinunternehmer gemäß § 19 UStG gilt auch für gemeinnützige Einrichtungen.

Die Überprüfung, betreffend die Umsatzgrenzen (32.500 DM Vorjahr und 100.00o DM Brutto-Gesamtumsatz im laufenden Kalenderjahr), bezieht sich auf den gesamten Unternehmensbereich des Museums und ist nicht auf den wirtschaftlichen Geschäftsbetrieb beschränkt. Umsätze, welche z. B. nach $\S 4 \mathrm{Nr}$. 11 bis 28 UStG steuerbefreit sind, bleiben jedoch gemäß § 19 Abs 3 UStG bei der Ermittlung des Gesamtumsatzes außer Ansatz.

Findet die Kleinunternehmerregelung keine Anwendung, weil der Bruttogesamtumsatz des Vorjahres mehr als 32.500 DM betragen hat, kann die steuerbegünstigte Körperschaft sich die Ermittlung der abziehbaren Vorsteuer erheblich vereinfachen, indem sie diese statt des Einzelnachweises mit einem Durchschnittsatz von sieben Prozent der steuerpflichtigen Umsätze ermittelt (Vorsteuerpauschale gemäß § 23 a UStG). Voraussetzung hierfür ist jedoch, daß der umsatzsteuerpflichtige Vorjahresumsatz (Netto-Einnahmen) unter 6o.0oo DM lag und die Anwendung dieser Regelung spätestens bis zum zehnten Tag nach Ablauf des ersten Voranmeldungszeitraums eines Kalenderjahres (bis zum 10.04.) gegenüber dem Finanzamt erklärt wird. Diese Erklärung entfaltet eine Bindungswirkung von fünf Jahren.

Die gleichen umsatzsteuerrechtlichen Konsequenzen ergeben sich zur Zeit in der Praxis, wenn der Förderverein den Museumsshop betreibt.

In diesem Zusammenhang sei mir eine kritische Anmerkung erlaubt. Meines Erachtens ist es fraglich, ob die Anwendung der Befreiungsvorschrift des $\S 4$ Nr. 20 a UStG bei Fördervereinen ge- 
setzeskonform ist. Denn bei den Fördervereinen handelt es sich um keine entsprechenden juristischen Personen des öffentlichen Rechts (Bund, Land, Gemeinde und Gemeindeverbände), noch betreiben diese selber die Museen, so daß entsprechende landesbehördliche Bescheinigungen nicht vorliegen.

Wird der Museumsshop von einer $\mathrm{GmbH}$ betrieben, ergeben sich grundsätzlich die gleichen umsatzsteuerrechtlichen Konsequenzen.

Abgrenzungsfragen, ob ein entsprechender Geschäftsvorfall dem Zweckbetrieb oder dem wirtschaftlichen Geschäftsbetrieb zuzurechnen ist, entscheiden sich ausschließlich nach den grundsätzlichen Bestimmungen der Abgabenordnung. Die umsatzsteuerrechtlichen Bestimmungen, insbesondere die Befreiungsvorschriften, sind für solche Abgrenzungsfragen nicht maßgeblich.

Bei dieser Abhandlung wurde bewußt nicht auf andere Steuerarten, z. B. die Lohnsteuer, eingegangen.

Weil es sich bei der steuerrechtlichen Behandlung von Museumsshops um ein sehr komplexes Thema handelt, und schon kleine Abweichungen $\mathrm{zu}$ den geschilderten Sachverhalt eine steuerrechtlich andere Würdigung zur Folge haben können, sollte vor Beginn der wirtschaftlichen Tätigkeit eine entsprechende steuerrechtliche Beratung stehen. 
Antonius J. Dommers

\title{
Organisations- und Trägerschaftsmodelle für Museumsshops
}

\author{
Formen betriebswirtschaftlicher Ausgliederung
}

Seit Mitte der 8oer Jahre kritisiert die Öffentlichkeit die mangelnde Effektivität, Effizienz, Kundenorientierung und Bürgernähe in der öffentlichen Verwaltung. Verstärkt wurde die Diskussion durch eine Krise der öffentlichen Haushalte. Es wurde vielfältig der Versuch unternommen, dem Problem durch Personaleinsparungen und Privatisierungen $\mathrm{zu}$ begegnen. In diesem Zusammenhang wurde auch versucht, die reine Hoheitsverwaltung zu trennen von den Dienstleistungsbereichen in der öffentlichen Hand. Spektakulär war die Bahn- und Postreform, die die Möglichkeiten der personellen Verschlankung über die Privatisierung im Auge hatte. Auch versprach man sich von dieser Maßnahme eine größere Flexibilität der Wirtschafts- und Dienstleistungsunternehmen und damit eine Kosteneinsparung und eine Entlastung der öffentlichen Hand. Viele andere Maßnahmen haben bei den Kommunen und Gebietskörperschaften dazu geführt, daß heute Dienstleistungsaufgaben in den unterschiedlichsten Rechtsformen erledigt werden.

$\mathrm{Zu}$ unterscheiden sind öffentlich-rechtliche und privatrechtliche Formen. Kultureinrichtungen können als Regiebetriebe, Eigenbetriebe, Stiftungen oder Zweckverbände in der öffentlichrechtlichen Form betrieben werden, wie auch in der Rechtsform einer $\mathrm{GmbH}$, eines eingetragenen Vereins oder einer Stiftung des privaten Rechts. 
$\mathrm{Zu}$ unterscheiden ist weiterhin die formale oder unechte Privatisierung von der materiellen Privatisierung. Während bei der formalen oder unechten Privatisierung eine Aufgabe ausgegliedert wird und weiterhin in kommunaler Trägerschaft bleibt (z. B. im Eigenbetrieb oder in der Eigen-GmbH), handelt es sich bei der materiellen Privatisierung um eine tatsächliche Abgabe von Aufgaben an private Dritte, die bis zur Veräußerung von öffentlichen Vermögenswerten führen kann.

Während bei der formalen oder unechten Privatisierung die Kommune oder die Dienstleistungskörperschaft weitgehend die Verantwortung in der Aufgabenerfüllung behält, wechselt bei der materiellen Privatisierung die Aufgabe von dem öffentlichen Bereich in den privaten Sektor. Im letzteren Falle ist die Einflußnahme der öffentlichen Hand schwach.

Schließlich kann die öffentliche Hand eine Aufgabe privaten Dritten zur Erledigung übertragen. Von der vertraglichen Regelung wird es abhängen, in welcher Weise die öffentliche Hand sich den Einfluß für die Aufgabenerledigung vorbehält.

Nachfolgend sollen die Möglichkeiten genannt werden, die bei der Betreibung von Museumsshops gelten können.

Museen werden überwiegend von der öffentlichen Hand unterhalten. Viele Gründe können dafür sprechen, daß der $\mathrm{Mu}$ seumsshop ausgegliedert und in einer anderen Rechtsform betrieben wird. So kann z. B. eine Verpachtung und Übertragung an einen Dritten zur wirtschaftlichen Nutzung gegen Zahlung einer Umsatz- oder Festpacht erfolgen. Mit der Übertragung der Führung des Museumsshops können auch andere Dienstleistungen verbunden sein, z.B. die Führung der Kasse zur Erhebung des Eintrittsgeldes, Aufsicht und Reinigung. Der Träger des Museums kann den Museumsbetrieb (Führung der Eingangskasse, Betrieb des Shops, Aufsicht und Reinigung) einer eigenen GmbH übertragen. Dieser Weg empfiehlt sich den gemeinnützigen Einrichtungen, die ihren wirtschaftlichen Geschäftsbetrieb strikt von dem gemeinnützigen Bereich trennen müssen. 
Ein im Rheinland seit Jahrzehnten bewährtes Modell soll vorgestellt werden

Einer der Vorreiter der Entwicklung, nämlich Kulturaufgaben in privatrechtlicher Form zu erledigen, war der Landschaftsverband Rheinland vor Jahren durch Gründung einer eigenen Gesellschaft, heute Rheinland-Verlag- und Betriebsgesellschaft (RVBG), mit der er sich ein betriebswirtschaftliches Instrumentarium für seine Kulturaufgaben schuf.

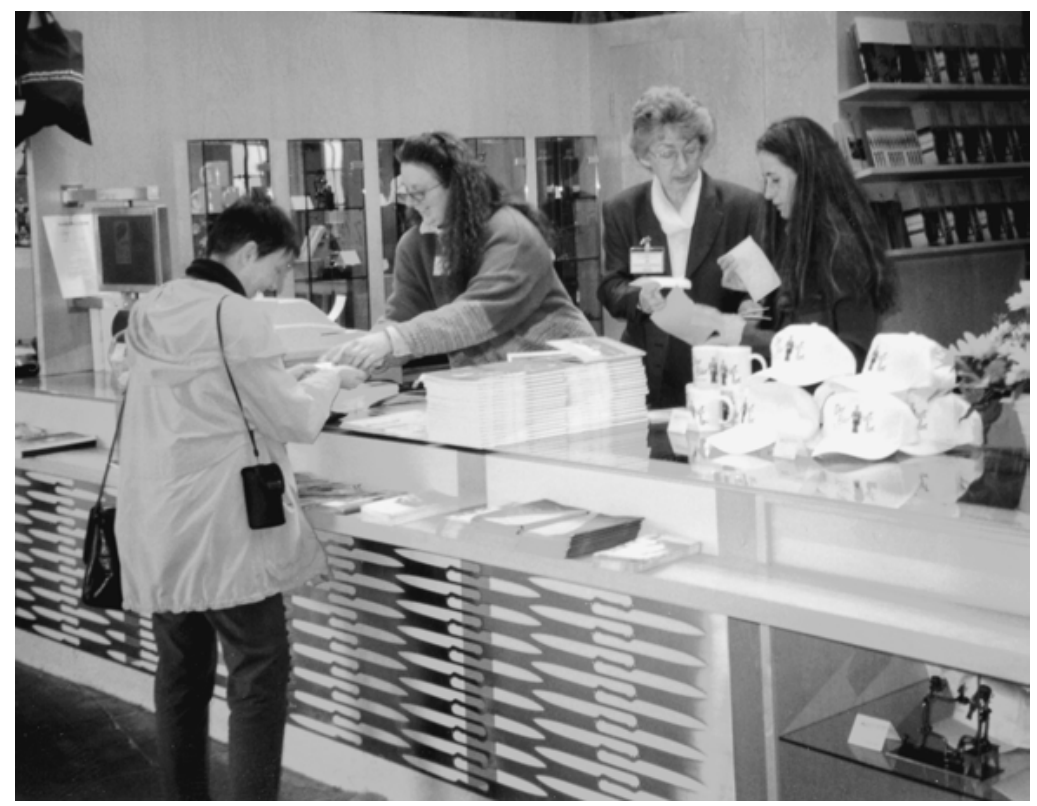

Rheinisches Industriemuseum, Gesenkschmiede Hendrichs, Solingen

Schon im Jahr 1958 konzentrierte der Landschaftsverband Rheinland in dem Rheinland-Verlag die verlegerische Tätigkeit und gab dort zunächst nur die Zeitschrift »neues Rheinland" für Landschaft und Kultur heraus. Im Jahr 1978 wurde eine Betriebsgesellschaft gegründet, die den Museen bei der Besucherbetreuung und bei der Abwicklung kaufmännischer Tätigkeiten behilflich sein sollte. Im Jahr 1987 wurden beide Gesellschaften zu einem Unternehmen zusammengeführt, das seit dieser Zeit den Namen Rheinland-Verlag- und Betriebgesellschaft des Landschaftsverbandes Rheinland $\mathrm{mbH}$ führt. 
In dem Unternehmenszweig "Verlag" sind bisher 1.700 Titel erschienen. Der Verlag führt 4o Publikations- und Schriftenreihen und betreut verlegerisch sechs Zeitschriften und Jahrbücher. Der Unternehmenszweig Verlag befindet sich am Ende der goer Jahre in einer Umstrukturierung. Der Landschaftsverband wird sich aus der verlegerischen Tätigkeit in diesem Zweig zurückziehen. Seine Publikationen wird er in Zukunft wie andere vergleichbare Kulturträger zusammen mit einem der vielen spezialisierten und bundesweit oder regional tätigen Verlage auf den Markt bringen.

In dem Unternehmenszweig "Museen« wird der Besucherservice derzeit für 22 Kultureinrichtungen besorgt. Es sind Museen des Landschaftsverbandes Rheinland und Museen anderer Träger. Darunter sind so bedeutende Einrichtungen wie der Archäologische Park Xanten, das Rheinische Freilichtmuseum Kommern und die Industriemuseen des Landschaftsverbandes Rheinland. Insgesamt 240 Mitarbeiterinnen und Mitarbeiter beschäftigt die RVBG in diesem Zweig. Mehr als 1 Mio. Museums- und Veranstaltungsbesucher werden derzeit jährlich gezählt.

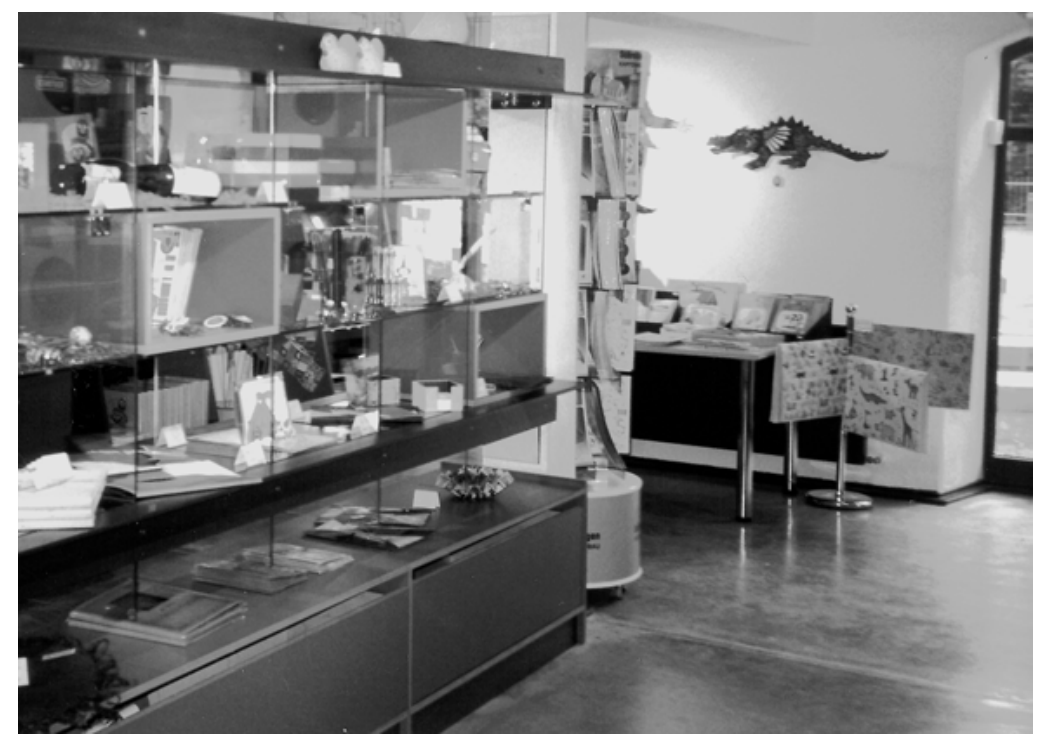

Rheinisches Industriemuseum, Alte Dombach, Bergisch Gladbach

Die Gesellschaft bietet einen Rundum-Service an, der die Werbung für den Museumsbesuch ebenso umfaßt wie die Kassenführung, den Verkauf von Museums-Souvenirs und Publikationen 
sowie die Aufsichts- und Reinigungsdienste und die Veranstaltungsdurchführung; also eine Gesellschaft mit breitem Spektrum, die sprichwörtlich rund um die Uhr tätig ist. Wenn die Mitarbeiter im Aufsichtsdienst am späten Nachmittag den Feierabend beginnen, nehmen die Nachtwachen den Dienst auf.

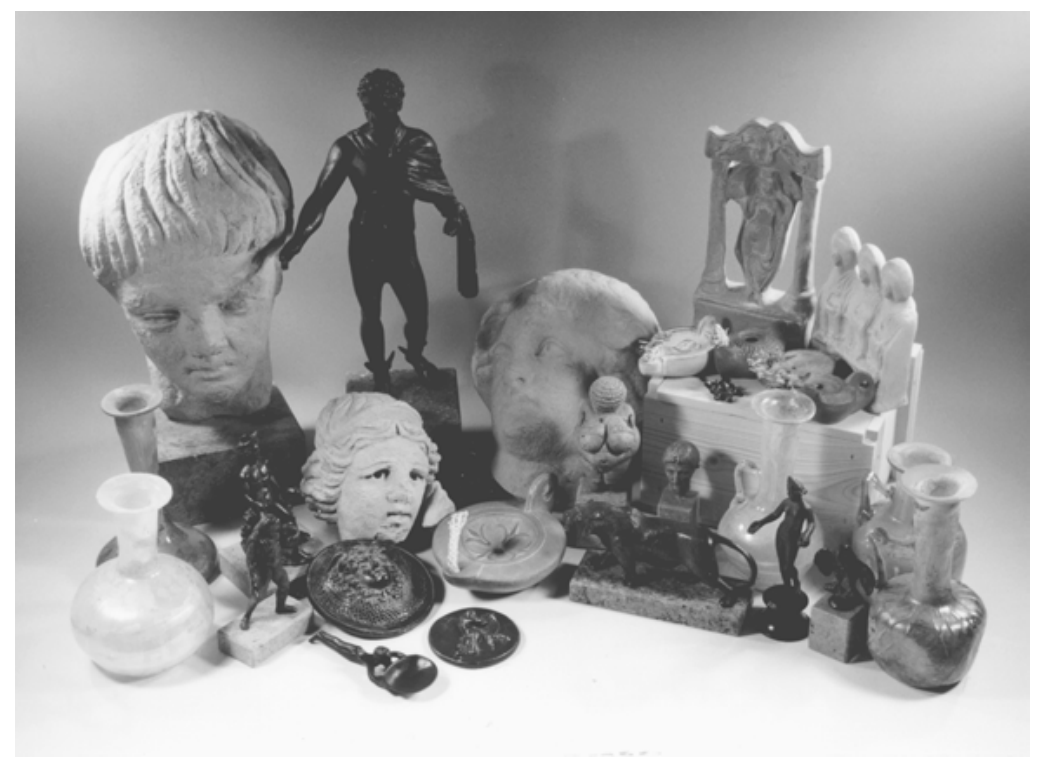

Artikel aus dem Verkaufssortiment der RVBG

Die Gesellschaft ist auf Expansion angelegt. Weitere Museen werden in nächster Zeit die Dienstleistungen der RVBG in Anspruch nehmen. Dabei erreichen alle Träger der betreuten Museen durch Synergieeffekte eine Kostensenkung bei der Betreuung der Besucher. Das reichhaltige Verkaufssortiment von derzeit nahezu 800 Artikeln weitet sich ständig aus. In enger Zusammenarbeit mit den Wissenschaftlern der betreuten Museen hat die RVBG bisher 200 eigene Artikel entwickelt. Jährlich kommen zahlreiche Artikel hinzu.

Der Dienst für den Besucher ist personalaufwendig, und so besetzt die RVBG immer wieder neue Arbeitsplätze, wenn es um die Betreuung neuer Museen oder die Durchführung von Veranstaltungen geht. Gerne in Anspruch genommen wird auch die Organisation und Durchführung von Museums-Gruppenfahrten in die betreuten Museen. 


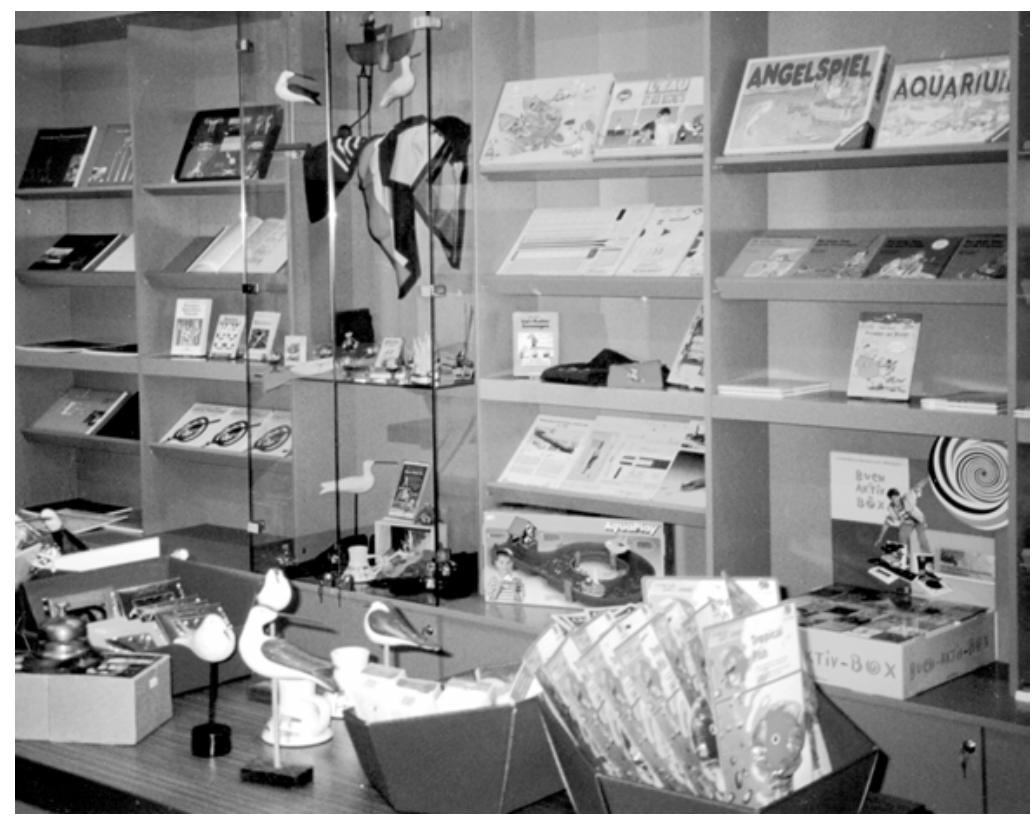

Deutsches Museum der Binnenschiffahrt, Duisburg-Ruhrort

Alleiniger Gesellschafter der RVBG ist der Landschaftsverband Rheinland. Ein Verwaltungsrat kontrolliert die Geschäftsführung und gibt die geschäftspolitischen Ziele der Gesellschaft vor. Verwaltungsorganisatorisch ist die RVBG der Abteilung für landschaftliche Kulturpflege und Umweltschutz des Landschaftsverbandes Rheinland zugeordnet, der auch die landschaftsverbandseigenen Museen unterstehen.

Eine pauschale Empfehlung für ein bestimmtes Trägerschaftsmodell ist nicht möglich. $\mathrm{Zu}$ berücksichtigen sind stets die komplexen wirtschaftlichen und steuerrechtlichen Auswirkungen einer bestimmten Wahl. Selbstbewirtschaftung oder Konzessionsvergabe an Dritte sind die grundsätzlichen Vorentscheidungen, die zu treffen sind. Bei der Selbstbewirtschaftung bleibt die Aufgabe und auch das Risiko der Bewirtschaftung des Museumsshops beim Träger. Ein Vorteil kann darin gesehen werden, daß der Träger die umfassende Einflußnahme und Kontrolle behält.

Bei der Konzessionsvergabe wird ein Dritter beteiligt, der im Wege der Pacht auf der Grundlage eines Vertrages in dem $\mathrm{Mu}$ seumsshop tätig wird. Ein Risiko für die Wirtschaftlichkeit entfällt in diesem Falle für den Museumsträger, er kann mit einer festen 


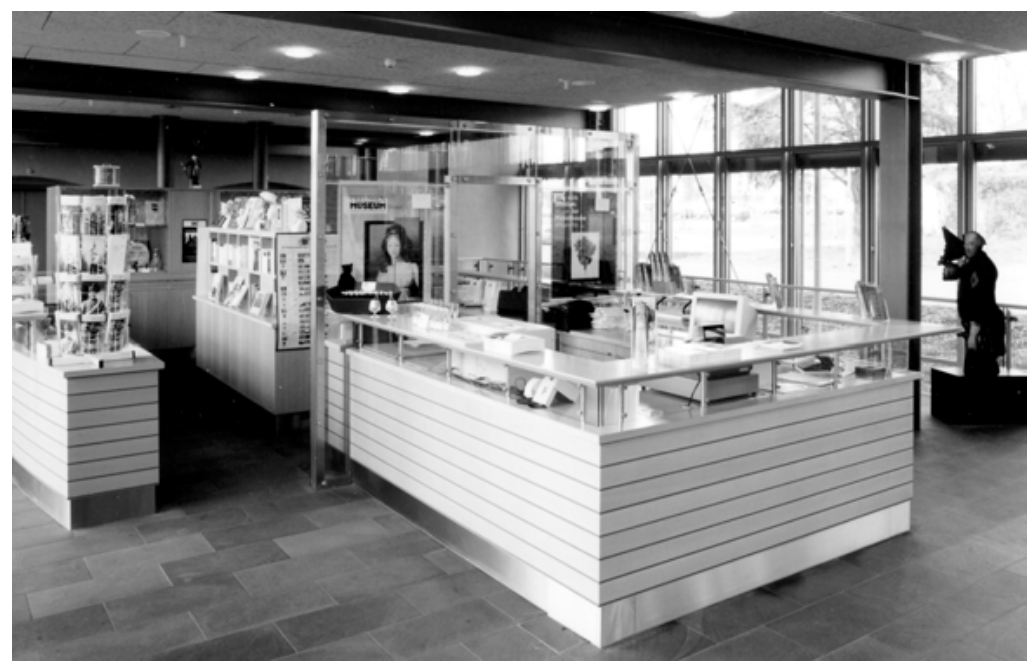

Preußen Museum Nordrhein-Westfalen, Standort Wesel

Einnahme aus der Verpachtung rechnen. Bei der Vertragsformulierung ist darauf zu achten, daß der Museumsträger sich angemessene Einflußrechte hinsichtlich des Shop-Sortiments und der Shop-Führung vorbehält.

Eine umfassende Bestandsaufnahme ist unumgänglich, bevor die Entscheidung für den Regiebetrieb, Eigenbetrieb, Zweckverband, die Stiftung, GmbH, den eingetragenen Verein oder die Übertragung an einen externen Pächter erfolgt. Aus der Erfahrung kann als Faustregel gelten, daß ein nennenswerter wirtschaftlicher Erfolg z. B. bei Verselbständigung des Shops erzielt werden kann, wenn bei einem angemessenen Eintrittsentgeld jährlich etwa 100.00o Besucher gezählt werden. Eintrittsgelder, Erlöse aus Warenverkäufen und Erlöse aus der Vermietung von Räumen unter der Durchführung von Veranstaltungen dürften dann den Aufwand decken, der durch den Shopbetrieb anfällt und schließlich einen Überschuß erzielen lassen. In diesem Zusammenhang ist es ratsam, die eingehende Beratung der bereits auf diesem Gebiet tätigen Unternehmen zu suchen. 


\section{Literatur}

Deutscher Städtetag (1996): Neue Rechtsformen für Kultureinrichtungen, Reihe 6, Heft 22, Köln.

Blanke, Thomas/Trümmer, Ralf (Hg.) (1998): Handbuch Privatisierung, Baden-Baden.

Küfner-Schmitt, Irmgard/Kulka, Michael (1998): Rechtliche Grundlagen der Privatisierung von Dienstleistungen im Museum, Handbuch des Museumsrechts 8, Berliner Schriften zur Museumskunde, Opladen. 
Anhang:

Die wesentlichen Unterschiede zwischen Regiebetrieb, Eigenbetrieb und GmbH

\begin{tabular}{|c|c|c|c|}
\hline & Regiebetrieb & Eigenbetrieb & GmbH \\
\hline $\begin{array}{l}\text { Rechtliche Ver- } \\
\text { hältnisse }\end{array}$ & $\begin{array}{l}\text { keine eigene Rechts- } \\
\text { persönlichkeit; Teil } \\
\text { des Gemeindevermö- } \\
\text { gens }\end{array}$ & $\begin{array}{l}\text { keine eigene } \\
\text { Rechtspersönlich- } \\
\text { keit; Sondervermö- } \\
\text { gen der Gemeinde; } \\
\text { Gründung muß } \\
\text { gemeinderechtlich } \\
\text { zulässig sein }\end{array}$ & $\begin{array}{l}\text { juristische Person; } \\
\text { handelsrechtlich und } \\
\text { steuerrechtlich eigen- } \\
\text { ständiges Rechtssub- } \\
\text { jekt; Gründung muß } \\
\text { gemeinderechtlich } \\
\text { zulässig sein }\end{array}$ \\
\hline Mindestkapital & keines & keines & $50.000 \mathrm{DM}$ \\
\hline $\begin{array}{l}\text { Organe und deren } \\
\text { Zuständigkeiten }\end{array}$ & $\begin{array}{l}\text { keine besondere Or- } \\
\text { ganisationsstruktur } \\
\text { und damit auch keine } \\
\text { besonderen Organe }\end{array}$ & $\begin{array}{l}\text { Werkleitung } \\
\text { Werkausschuß } \\
\text { Gemeindedirektor } \\
\text { Gemeinderat }\end{array}$ & $\begin{array}{l}\text { Geschäftsführer } \\
\text { Aufsichtsrat (fakulta- } \\
\text { tiv oder zwingend) } \\
\text { Gesellschafterver- } \\
\text { sammlung }\end{array}$ \\
\hline $\begin{array}{l}\text { Geschäftsführung } \\
\text { und Vertretung }\end{array}$ & $\begin{array}{l}\text { Gemeindedirektor ist } \\
\text { gesetzlicher Vertreter }\end{array}$ & $\begin{array}{l}\text { Werkleitung nur in- } \\
\text { soweit vertretungsbe- } \\
\text { fugt, als ihr auch Ent- } \\
\text { scheidungskompetenz } \\
\text { zukommt; ansonsten } \\
\text { Gemeindedirektor }\end{array}$ & $\begin{array}{l}\text { Geschäftsführung } \\
\text { unbegrenzt nach au- } \\
\text { ßen vertretungsbe- } \\
\text { rechtigt }\end{array}$ \\
\hline Leitungsstruktur & $\begin{array}{l}\text { lange Entschei- } \\
\text { dungswege und par- } \\
\text { zellierte Zuständig- } \\
\text { keiten }\end{array}$ & $\begin{array}{l}\text { kürzere Entschei- } \\
\text { dungswege und weni- } \\
\text { ger parzellierte } \mathrm{Zu} \text { - } \\
\text { ständigkeiten }\end{array}$ & $\begin{array}{l}\text { kurze Entschei- } \\
\text { dungswege und Ge- } \\
\text { samtzuständigkeit der } \\
\text { Geschäftsführung: } \\
\text { Identität von Verant- } \\
\text { wortung und Ent- } \\
\text { scheidung }\end{array}$ \\
\hline Personalwesen & $\begin{array}{l}\text { eingebunden in das } \\
\text { öffentliche Dienst- } \\
\text { recht; die Gemeinde } \\
\text { ist Arbeitgeber der } \\
\text { Beschäftigten }\end{array}$ & $\begin{array}{l}\text { eingebunden in das } \\
\text { öffentliche Dienst- } \\
\text { recht; die Gemeinde } \\
\text { ist Arbeitgeber der } \\
\text { Beschäftigten; eigener } \\
\text { Stellenplan; be- } \\
\text { schränkt eigene Per- } \\
\text { sonalwirtschaft } \\
\end{array}$ & $\begin{array}{l}\text { Abschluß von indivi- } \\
\text { duellen Anstellungs- } \\
\text { verträgen; Flexibilität } \\
\text { auch bei Anwendung } \\
\text { von BAT und BMT-G } \\
\text { eigene Personalwirt- } \\
\text { schaft }\end{array}$ \\
\hline $\begin{array}{l}\text { Personalvertre- } \\
\text { tung / Mitbe- } \\
\text { stimmung }\end{array}$ & \multicolumn{2}{|c|}{$\begin{array}{l}\text { unterliegen dem Landespersonalvertretungs- } \\
\text { gesetz; Personalrat }\end{array}$} & $\begin{array}{l}\text { gemäß Betriebsver- } \\
\text { fassungsgesetz; Be- } \\
\text { triebsrat }\end{array}$ \\
\hline Haftung & \multicolumn{2}{|c|}{$\begin{array}{l}\text { Gemeinde haftet uneingeschränkt für die } \\
\text { Verbindlichkeiten }\end{array}$} & $\begin{array}{l}\text { Haftung beschränkt } \\
\text { auf das Gesellschafts- } \\
\text { vermögen }\end{array}$ \\
\hline
\end{tabular}




\begin{tabular}{|c|c|c|c|}
\hline & Regiebetrieb & Eigenbetrieb & GmbH \\
\hline $\begin{array}{l}\text { Wirtschaftspla- } \\
\text { nung }\end{array}$ & Haushaltsplan & \multicolumn{2}{|c|}{$\begin{array}{l}\text { eigener Wirtschaftsplan (außerhalb des all- } \\
\text { gemeinen Haushalts) }\end{array}$} \\
\hline Finanzierung & $\begin{array}{l}\text { im Rahmen des Ge- } \\
\text { samthaushaltsplans }\end{array}$ & $\begin{array}{l}\text { beschränkt eigene } \\
\text { Kreditwirtschaft }\end{array}$ & $\begin{array}{l}\text { eigene Kreditwirt- } \\
\text { schaft }\end{array}$ \\
\hline $\begin{array}{l}\text { Rechnungswesen } \\
\text { und } \\
\text { Rechnungslegung } \\
\text { a) Ausführung des } \\
\text { Wirtschafts- } \\
\text { planes }\end{array}$ & $\begin{array}{l}\text { kassenmäßiger Voll- } \\
\text { zug durch kamerali- } \\
\text { stische Verbuchung }\end{array}$ & \multicolumn{2}{|c|}{ kaufmännische doppelte Buchführung } \\
\hline b) Abrechnung & $\begin{array}{l}\text { Jahresrechnung nach } \\
\text { Gemeinderecht }\end{array}$ & \multicolumn{2}{|c|}{$\begin{array}{l}\text { Jahresabschluß (Bilanz/Gewinn- und Ver- } \\
\text { lustrechnung/Anhang), Lagebericht }\end{array}$} \\
\hline $\begin{array}{l}\text { Prüfungswesen } \\
\text { a) örtliche } \\
\text { Prüfung }\end{array}$ & \multirow{2}{*}{\multicolumn{2}{|c|}{$\begin{array}{l}\text { Rechnungsprüfungsausschuß, Rechnungs- } \\
\text { prüfungsamt der Gemeinde } \\
\text { Gemeindeprüfungsamt }\end{array}$}} & $\begin{array}{l}\text { Prüfung gemäß Han- } \\
\text { delsgesetzbuch }\end{array}$ \\
\hline $\begin{array}{l}\text { b) überörtliche } \\
\text { Prüfung }\end{array}$ & & & $\begin{array}{l}\text { Bestätigungsprüfung } \\
\text { durch die Gemeinde }\end{array}$ \\
\hline Steuerrecht & \multicolumn{2}{|c|}{$\begin{array}{l}\text { ist steuerlich relevant, wenn ein Betrieb ge- } \\
\text { werblicher Art im Sinne des § 9 Abs. } 1 \text { des } \\
\text { Körperschaftssteuergesetzes vorliegt und un- } \\
\text { terliegt dann grundsätzlich allen Steuerarten } \\
\text { (Körperschaftssteuer, Gewerbesteuer, Um- } \\
\text { satzsteuer, Vermögenssteuer usw.) }\end{array}$} & $\begin{array}{l}\text { ist steuerlich stets re- } \\
\text { levant und unterliegt } \\
\text { demgemäß grundsätz- } \\
\text { lich allen Steuerarten }\end{array}$ \\
\hline $\begin{array}{l}\text { Bindung an VOB/ } \\
\text { VOL }\end{array}$ & ja & $\begin{array}{l}\text { landesrechtlich un- } \\
\text { terschiedlich (in NRW } \\
\text { z.B. keine Anwen- } \\
\text { dungspflicht, ausge- } \\
\text { nommen Anwendung } \\
\text { ist durch Ortsrecht } \\
\text { oder Dienstanwei- } \\
\text { sung vorgeschrieben } \\
\text { oder wird vom Zu- } \\
\text { schußgeber verlangt) }\end{array}$ & $\begin{array}{l}\text { VOB: künftig nach } \\
\text { EU-Recht } \\
\text { VOL: nein }\end{array}$ \\
\hline $\begin{array}{l}\text { Beteiligungsmög- } \\
\text { lichkeit Dritter }\end{array}$ & nein & nein & ja \\
\hline
\end{tabular}

Quelle: DST-Beiträge zur Bildungs- und Kulturpolitik, Reihe C, Heft 22 


\section{Der Museumsshop in Trägerschaft eines Fördervereins}

Vorgestellt sei hier der Museumsshop des städtischen Kunstmuseums in der Alten Post in Mülheim an der Ruhr, der seit dem 6. Dezember 1997 vom Förderkreis für das Museum in ehrenamtlicher Trägerschaft betreut wird und von diesem Verein auch eingerichtet wurde. Die Entscheidung, die Trägerschaft einem ehrenamtlichen Verein zu übertragen, lag nicht zuletzt in der Vorgeschichte des Museums begründet.

\section{Zum Museum}

Das Kunstmuseum in der Alten Post ist ein von der Stadt getragenes Haus, dessen Sammlungsschwerpunkt Kunst des 20. Jahrhunderts ist. Es wurde erst im August des Jahres 1994 in einem komplett umgestalteten Gebäude wieder neu eröffnet, nachdem es zuvor seit seiner Gründung $\mathrm{zu}$ Beginn des Jahrhunderts häufig wechselnde und eher bescheidene Räumlichkeiten zur Verfügung hatte. Seither befindet es sich im ehemaligen Hauptpostamt der Stadt, einem Gebäude aus dem ausgehenden 19. Jahrhundert, das mit seinem neogotischen Baustil eines der wenigen noch originalen historischen Gebäude der Stadt ist. Nach Vorgaben des Denkmalschutzes wurde dort ein Museum eingerichtet, das - auch in Hinsicht auf die klimatischen Bedingungen - modernen Anforderungen für Präsentation und Unterbringung entspricht. Die 
Denkmalwürdigkeit dieses Bauwerks war jedoch auch der Grund, daß das Foyer des Museums, die alte Schalterhalle der Post, so wenig wie möglich verändert wurde, um wenigstens hier noch den alten Charakter eines behördlichen Gebäudes des 19. Jahrhunderts zu erhalten. Nicht zuletzt deshalb wurde es im Vorfeld der Baumaßnahmen unterlassen, an eine etwaige spätere Planung eines Verkaufsraumes zu denken, der dort seine Heimat finden müßte, und man verzichtete auf bauliche Vorgaben für einen solchen Bereich.

\section{Warum ein Museumsshop?}

Mit Eröffnung des Museums im August 1994 verstärkte sich jedoch der Wunsch, einen Museumsshop im Foyer einzurichten. Neben der Attraktivierung des Museums selbst ließ die Lage der Alten Post in der Fußgängerzone der Innenstadt darauf hoffen, daß ein solcher Museumsshop auch für sLaufkundschaft` von Interesse sein könnte.

Durch die Erweiterung der Angebotspalette für die Besucher sollten neben Katalogen und Postkarten aus dem Sammlungsbestand auch weitere Kunstpostkarten, Kalender und Grußkarten angeboten werden. Durch Nachfragen von Gästen des Hauses wurde sehr bald deutlich, daß ein solcher Bedarf vorhanden war. Zusätzlich sollte eine optische Attraktivierung des Museums und seiner etwas schwierigen Eingangssituation nach außen hin erreicht werden. Diese deutlichere optische Präsenz sollte dann auch eine sLockfunktion besitzen, die nicht zuletzt zur Überwindung von >Museumszugangshemmungen - sprich: Schwellenangst - führen kann.

\section{Konzeption der Maßnahme}

Die Besucherzahlen des Kunstmuseums beziffern sich nach der Eröffnung auf 12.000 bis 15.000 Besucher im Jahr, wodurch einige wirtschaftliche Formen solcher Verkaufsbereiche indirekt ausgeschlossen schienen. Bei den zu erwartenden Erträgen hätte es sich für einen freien Anbieter kaum gelohnt, einen solchen Shop zu betreiben. Daher war bald klar, daß der Museumsshop in eigener Regie durchgeführt werden muß. 


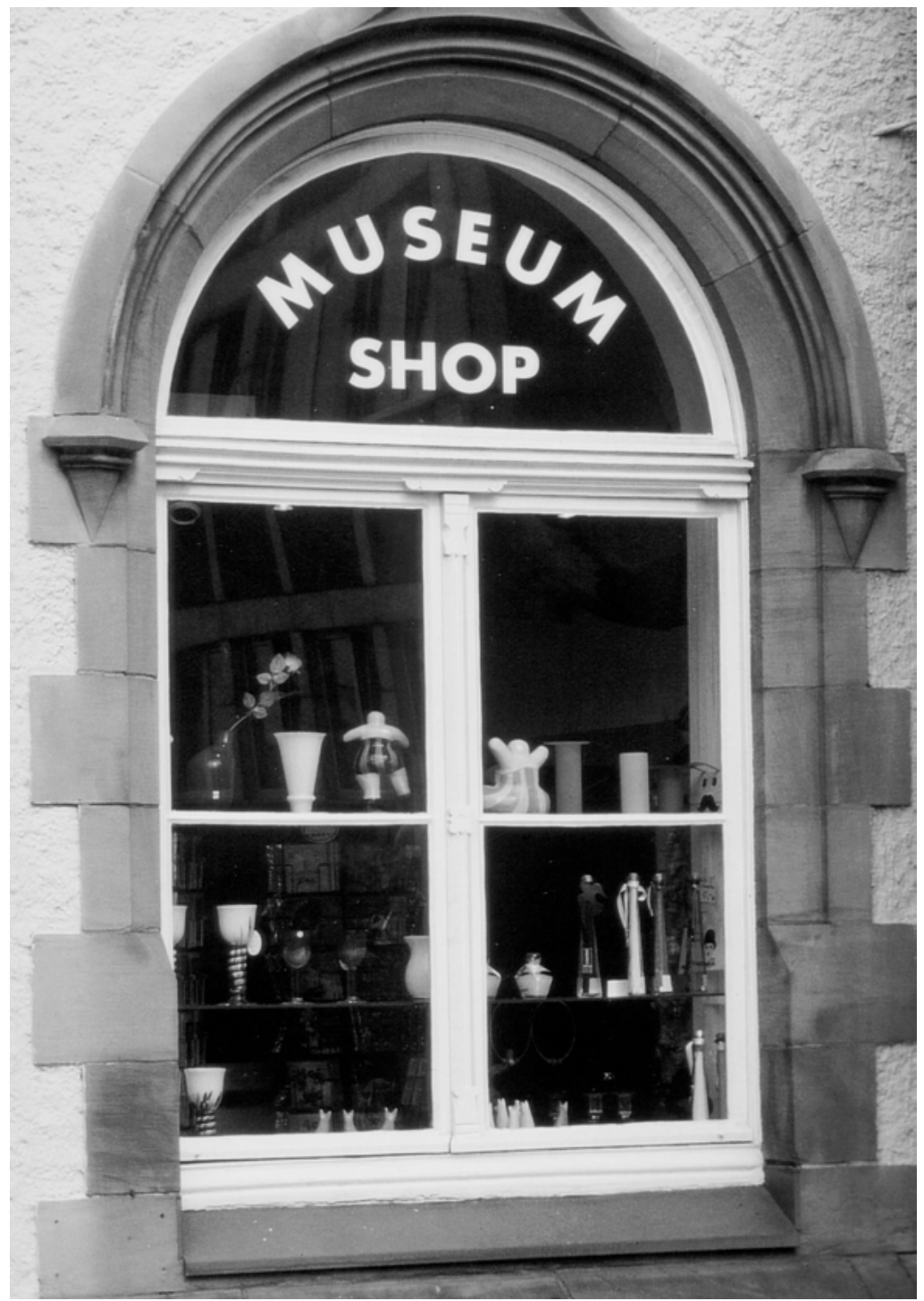

Die Frage, wer den Museumsshop realisiert, führte zu drei wichtigen Gesichtspunken: Wie kann ein solcher zusätzlicher Verkauf vorfinanziert werden, wie fließen die Gewinne dem Museum wieder zu, und wie soll er personell durchgeführt werden?

Zunächst einmal mußte die Grundeinrichtung des Museumsshops finanziert werden, was das Museum mit städtischen Mitteln nicht bestreiten konnte. Zum zweiten sollte gesichert sein, daß die 


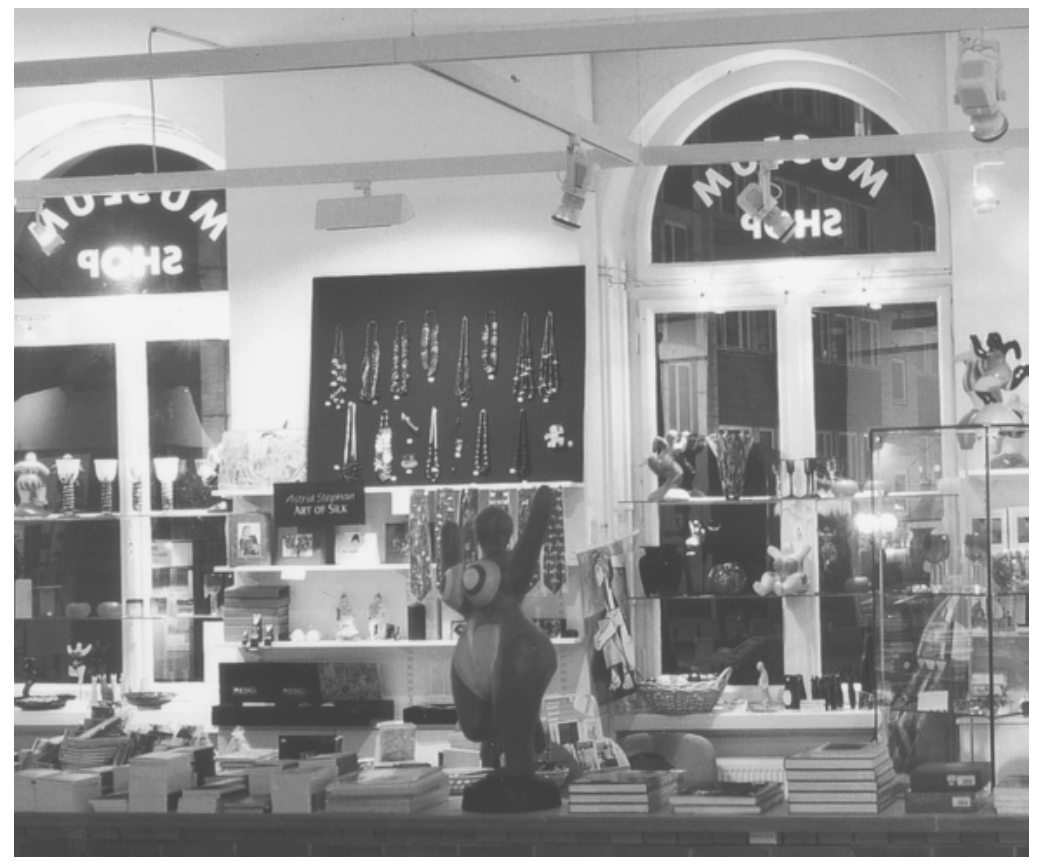

Einnahmen des Museumsshops nur zum Zweck der Unterstützung des Museums eingesetzt werden, speziell dort zu Zwecken der Sammlungserweiterung oder für besondere Ausstellungsprojekte. Da das Museum der Stadt Mülheim an der Ruhr gemeinsam mit allen anderen Kulturinstituten seit dem 1. Januar 1996 dem städtischen Kulturbetrieb, einer eigenbetriebsähnlichen Einrichtung, zugeordnet ist, ist zwar die Zuordnung in das kameralistische System nicht mehr gegeben, jedoch wären Einnahmen nicht zwangsläufig nur zur Realisierung dieser zusätzlichen Förderzwecke einzusetzen gewesen. Darüber hinaus sollte die Gestaltung des $\mathrm{Mu}$ seumsshops mit seiner Angebotspalette kontinuierlich aufgebaut, ständig erweitert und aktualisiert werden. Diese verantwortungsvolle Aufgabe verlangt entsprechenden Zeitaufwand durch Messebesuche, Aquisition und Kontakte zu anderen Museumsshops, was die relativ kleine Mannschaft der städtischen Museen in Mülheim nicht zusätzlich leisten konnte. Und nicht zuletzt stand die Frage im Raum, wer verkauft die Waren und bringt sie »an Mann und Frau«? Denn bei dieser Frage war nicht nur der Zeitaufwand ein entscheidender Faktor, sondern auch die Betreuung des $\mathrm{Mu}$ seumsshops durch besonders motiviertes Personal. Ehrenamtliche 
Mitarbeiter und Mitarbeiterinnen sind besonders engagiert in ihrer Tätigkeit, da sie sich von vornherein mit dem Museum, seinen Inhalten und der Realisierung des Museumsshops identifizieren. Daher bestand - in Analogie zu anderen Museumsshops, vor allen Dingen zu dem des Von der Heydt-Museums in Wuppertal - schon recht früh der Wunsch, die Betreuung und Realisierung verantwortlich in die Hände eines Förderkreises bzw. Museumsvereins zu legen.

Die bestehenden Erfahrungen mit dem hiesigen Förderkreis für das Museum waren bereits lange vor der Wiedereröffnung des Museums 1994 von besonderen kaufmännischen Aktivitäten bestimmt. Der Förderkreis hatte ein Faksimile der "Suite Vollard « von Picasso herausgegeben, das in den letzten Jahren dem Museum nicht unerhebliche Einnahmen beschert hatte, mittels derer Kunstwerke für den Sammlungsbestand erworben werden konnten. Dazu zählen ein Ölgemälde von Emil Schumacher, drei Aquarelle von Georg Baselitz, sechs Arbeiten auf Papier von A.R. Penck und vier Arbeiten auf Papier von Markus Lüpertz. Der kaufmännische Geist ist in diesem Förderverein sehr lebendig, und so fand der Vorstand nach einigem Suchen zwei engagierte Personen, die sich bereit erklärten, dieses schwierige Unterfangen auf sich zu nehmen. Nach diesem ersten - und wie ich meine wichtigsten - Schritt zur `Existenzgründung gelang die konkrete Umsetzung des Museumsshops innerhalb von einem knappen Jahr relativ zügig.

\section{Realisierung des Museumsshops}

Der Museumsshop wurde in der Schalterhalle angesiedelt, und zwar in dem Bereich hinter einem der denkmalgeschützten Postschalter, wobei dieser Bereich ohne Zugang in die Ausstellungsräume des Museums genutzt werden kann. Der Verkaufsraum dort ist ca. $50 \mathrm{~m}^{2}$ groß. Um den Bereich freundlicher zu gestalten, wurde die Türzugangsbreite dort erweitert, ein neuer Bodenbelag eingesetzt, der diesen Bereich abgrenzt und gleichzeitig zusammenfaßt, und es wurden Einrichtungsmöbel wie Kassentheke, Vitrinen und Schrankwandsysteme zwischen den Fenstern in Auftrag gegeben und paßgenau für diesen Bereich hergestellt. Die Gestaltung der Fenster wurde gleichzeitig als Schaufenster nach draußen umgesetzt und mit einer zusätzlichen Beleuchtung versehen, so 
daß auch außerhalb der Öffnungszeiten des Museums für Passanten ein Blick in die Auslagen möglich ist.

Dann erfolgte die Grundeinrichtung des Museumsshops mit den ersten Artikeln, wobei hier zunächst die Möglichkeit von Kommissionsware genutzt werden konnte. Als eine der ersten eigenen Maßnahmen wurde eine Weinflasche mit einem Motiv des Museumsbestandes bedruckt und zur Eröffnung des Shops angeboten. Die Einrichtung des Museumsshops hat den Förderkreis für das Museum etwa 60.00o DM Investition gekostet, dazu kamen weitere investive Maßnahmen der Stadt, die die Erweiterung des Türzugangs zu diesem Bereich noch einmal mit ungefähr 12.000 DM finanzieren mußte.

\section{"Fast ein Jahr Museumsshop»}

Die Produktpalette hat sich langsam entwickelt und sich zunächst an bestehenden Museumsshops orientiert. Die Vorgabe, den Artikelfundus auf mindestens 20 Prozent museumseigene Artikel zu konzentrieren, ist noch nicht erfüllt; die wenigen Artikel, die sich auf Sammlungsbestände des Museums beziehen, sind zur Zeit unsere Faksimile-Mappe der "Suite Vollard« von Picasso und dem einen oder anderen Museumsmotiv, das sich auf Weinflaschen, Fotoboxen oder Kalendern befindet. Bei zehn bis zwölf Wechselausstellungen im Jahr ist es nicht möglich, sich auf die aktuellen Präsentationen zu konzentrieren, da diese allzu schnellebig sind und die Artikel nicht genügend Absatz finden würden. Der Schwerpunkt soll auch in Zukunft daher die Orientierung am Sammlungsbestand sein, dessen >Vermarktung in den nächsten Jahren weiter umgesetzt wird. Aus verschiedenen Gründen werden die Kataloge des Museums zum Bestand und zu den Ausstellungen nicht im Museumsshop verkauft, da hierfür im Museumsetat ein fester Budgetbetrag vorhanden ist, der durch das Ausgliedern dieser Beträge im Budget fehlen würde, und über eine solche Gegenverrechnung mit dem Museumsshop noch keine konkreten vertraglichen Grundlagen gefunden worden sind.

Der Personalstab unseres Museumsshops besteht im Moment aus zwei ehrenamtlich/hauptamtlichen Mitarbeiterinnen, die den Shop und seine Umsetzung betreuen, einem ehrenamtlichen Mitarbeiter, der die Buchhaltung des Museumsshops übernommen hat, und zwanzig ehrenamtlichen Verkaufshilfen, so daß während 


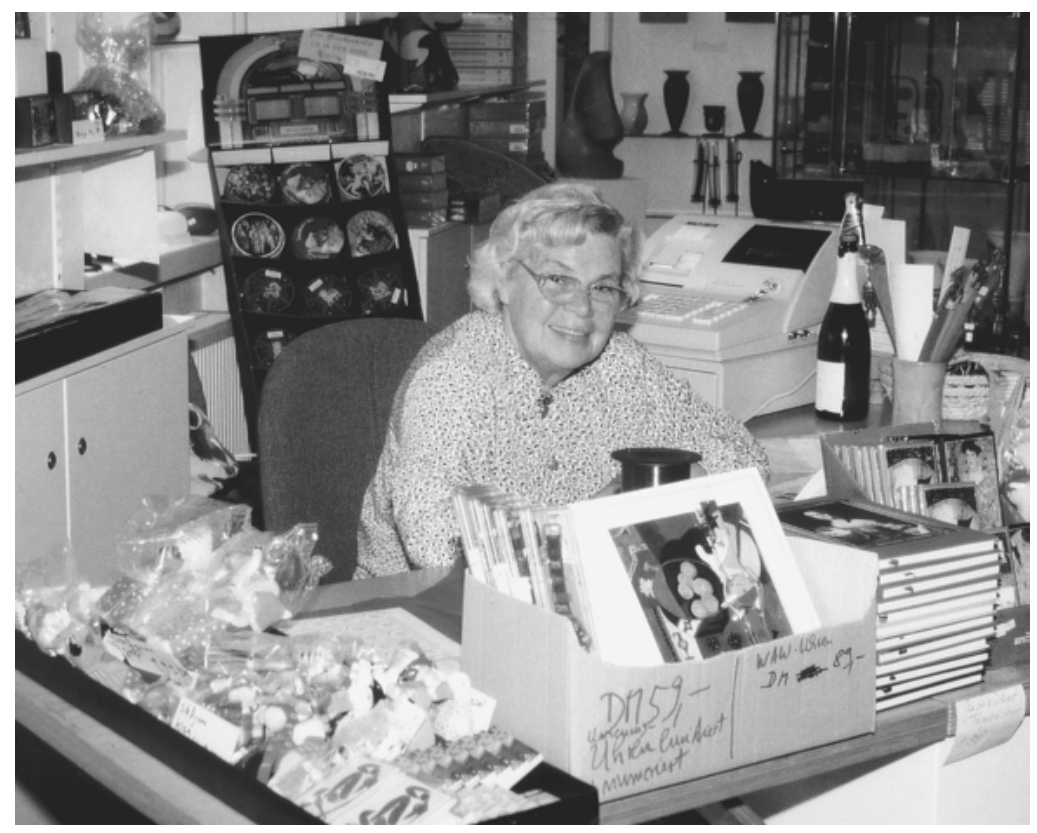

der Öffnungszeiten des Museums der Museumsshop ständig geöffnet sein kann.

Die Auswirkungen, die sich seit dem 6. Dezember 1997 für das Museum ablesen lassen, sind allesamt positiv - bis auf die rechtliche Situation und den Status, den der Museumsshop beim Finanzamt bekommen hat. Leider ist der Mülheimer Museumsshop immer noch nicht als Zweckbetrieb vom Finanzamt anerkannt, so daß Gewerbesteuer, Umsatzsteuer und Körperschaftssteuer für die Erträge des Museumsshops in Anrechnung gebracht werden. Zur Zeit wird eine vertragliche Vereinbarung erarbeitet, die dafür eine bessere Lösung finden soll. Die Attraktivierung für das Museum jedoch ist bereits erreicht, und es nimmt auch die Zahl der Besucher zu, die in Kenntnis des Museumsshops mal schnell vorbeischauen, um ein Geschenk oder etwas anderes zu suchen und dabei gleichzeitig die Informationen mitnehmen, welche Ausstellungen derzeit im Museum zu sehen sind. Im letzten Sommerschlußverkauf nämlich, zu dem etliche Kunden mehr in die Innenstadt der Stadt Mülheim gekommen sind, stiegen auch die Besucherzahlen des Museums - und dabei waren sicherlich viele, die niemals zuvor ihren Fuß in das Museum ihrer Stadt gesetzt haben. 
Am 20. Oktober 1998 wurde aus den ersten Gewinnen auch der erste Ankauf für den Sammlungsbestand des Museums getätigt. Gemeinsam mit den ehrenamtlichen Mitarbeiterinnen wurde ein Atelierbesuch bei einem jungen Mülheimer Künstler organisiert, der zu diesem Zeitpunkt eine erste Einzelausstellung im Kunstmuseum hatte. Gemeinsam mit Museumsleiterin, Künstler und den engagierten Damen des Museumsshops wurde eine sechsteilige Papierarbeit ausgewählt, die nun zum Sammlungsbestand des $\mathrm{Mu}$ seums zählt. Ein freudiger Abend für alle Beteiligten - konnte man endlich auch mit Händen greifen, wofür all' die Mühe sich gelohnt hat! 


\section{Die GmbH als Museumsshop-Betreiber}

Das Landesmuseum Koblenz hat 1981 - also vor 18 Jahren - den ersten Museumsshop seinen Besuchern angeboten. Getragen durch den 1977 gegründeten »Verein der Freunde und Förderer des Landesmuseums Koblenz e.V. « erreichten die wirtschaftlichen Aktivitäten bereits $1982 \mathrm{im}$ Kontext der Sonderausstellung »Vom Korn zum Brot« und dem damit verbundenen Betrieb einer Museumsbäckerei, die an sieben Wochentagen jeweils zwölf Stunden von gelernten Bäckern bedient wurde, Dimensionen, die zu intensiven Auseinandersetzungen mit dem Finanzamt Koblenz führen mußten. 1986 wurden deshalb die Aktivitäten erheblich reduziert, der Museumsshop geschlossen und Finanzmittel angespart, um die Einlage zur Gründung einer $\mathrm{GmbH}$ in Höhe von 50.000 DM zu erreichen.*

\section{Die proMuseum $\mathrm{GmbH}$}

Die proMuseum GmbH konnte schließlich am 20.4.1993 gegründet werden. Mit ihr war eine steuerrechtlich abgesicherte Basis geschaffen, um als Wirtschaftsförderungsgesellschaft für das Landesmuseum Koblenz geschäftliche Aktivitäten zu entwickeln; die finanziellen Erträge wurden wiederum dem Verein der Freunde und Förderer des Landesmuseums Koblenz e.V. zur satzungsgemä- 
ßen Verwendung zugeführt. Die proMuseum $\mathrm{GmbH}$ ist somit eine hundertprozentige Tochter des Fördervereins.

In $\S 2$ der $\mathrm{GmbH}$-Satzung ist der Umfang der geschäftlichen Aktivitäten der proMuseum $\mathrm{GmbH}$ definiert:

Gegenstand des Unternehmens ist der Betrieb eines Museumsshops und alle damit zusammenhängenden Geschäfte sowie die Herstellung von und der Handel mit museumsrelevanten Gegenständen. Die Gesellschaft kann alle darüber hinausreichenden Handlungen vornehmen, die mittelbar oder unmittelbar zur Förderung des Landesmuseums Koblenz geeignet sind.

Die Gesellschaft hat einen oder mehrere Geschäftsführer sowie einen Beirat, in welchem der Direktor des Landesmuseums Koblenz geborenes Mitglied ist. Aufgabe des Beirates ist es, die Geschäftsführung zu beraten. Er wählt aus seiner Mitte einen Vorsitzenden.

Eine jährliche Gesellschafterversammlung hat stattzufinden, auf ihr ist der Jahresabschluß vorzutragen und dem Geschäftsführer Entlastung zu erteilen. Die Satzung weist auch diejenigen Geschäfte aus, die der Zustimmung des Beirates bedürfen.

Bereits das Geschäftsjahr 1993, das erst am 1.5.1993 begann, wies einen erwirtschafteten Gewinn von ca. 16.500 DM aus, der in voller Höhe an den Förderverein überwiesen wurde.

\section{Das erste Betriebsjahr}

Der Betrieb über sechseinhalb Monate - das Museum schloß am 21.11.1993 für die Wintermonate 1993/1994 und zählte etwa 304.00o Besucher - hatte gezeigt,

- daß die Personalkosten mit fast 25.00o DM trotz der Minderlohnverträge in hohem Maße ergebnisreduzierend waren,

- daß der Arbeitsaufwand der Geschäftsführerin (auch im Minderlohnvertrag) durch Personaldisposition, Rechnungswesen, Einkäufe und Messebesuche, Verkaufstätigkeit an Wochenenden usw. erheblich ist,

- daß die Personaldisposition im Verkauf durch gesundheitliche und familiäre Beeinträchtigungen immer wieder zu kurzfristigen und arbeitsintensiven Umdisponierungen führte, 
- daß die Buchhaltung an eine Fachkraft im Lohnauftrag vergeben werden mußte,

- daß das Warensortiment auf Besucherwünsche und die Besucherstrukturen abzustimmen ist und in beachtlichem Maße von den Themen der Sonderausstellungen beeinflußt wird,

- daß preiswerte Waren dennoch qualitativ ansprechend sein müssen und hochwertige Waren vorsichtig $\mathrm{zu}$ disponieren sind. Warenübernahmen im Zusammenhang mit Sonderausstellungen in Kommission zeigten sich als finanzentlastend und risikoreduzierend.

Die bereits 1993 gemachten Erfahrungen bestätigten sich im Geschäftsjahr 1994 weitgehend. Umsatzsteigerungen waren durch die anhaltende wirtschaftliche Rezession kaum möglich. Auch das seit Mitte September in der Sonderausstellung "Kaffee, Mokka, Mukkefuck« betriebene "Wiener Kaffeehaus« konnte durch die entstehenden Personalkosten keine hohen Gewinne aufweisen. Infrastrukturelle Probleme durch die historische Baustruktur und Architektur der Festung Ehrenbreitstein beeinträchtigten die Arbeitsabläufe in überdurchschnittlichem Maße.

Eine eingehende Prüfung der drei rheinland-pfälzischen Landesmuseen durch den Rechnungshof Rheinland-Pfalz im Jahre 1993 diente u.a. auch der Prüfung, welche Leistungen und Arbeiten kostengünstiger erhältlich sind, einschließlich der Vergabe an private Unternehmen. Auch die Frage, ob eine andere Trägerschaftsform denkbar und rechenbar sei, wurde mit den Prüfern diskutiert.

Nach nunmehr sechs Betriebsjahren zeigten sich für uns die folgenden Kriterien, die an einem anderen Platz mit anderen Konstellationen und Rahmenbedingungen durchaus zu modifizieren sind.

\section{Wie wird ein Shop erfolgreich betrieben?}

Wollen Sie erfolgreich einen Museumsshop in Deutschland betreiben, dann haben Sie aus meiner Sicht möglichst viele der folgenden Kriterien und Vorgaben zu erfüllen: 
1. Die Sammlungen oder Ausstellungen sollten kunsthistorisch oder kunstgewerblich ausgerichtet sein. Besucher solcher $\mathrm{Mu}$ seen sind an den Objekten vielseitig interessiert, sammeln häufig selbst, kaufen die neueste Fachliteratur zu den Themen, die sie interessieren, umgeben sich gerne mit Signalen, die anderen Zeitgenossen mitteilen, daß man dabei sein will. Diese Besucher verdienen überdurchschnittlich und suchen ihre Geschenke für andere gerne in solchen Shops. Das Bewußtsein, das Museum indirekt zu unterstützen, ist ausgeprägt. Solche Museen oder Ausstellungen sprechen den Adressaten emotional positiv an. Exponate und Shopartikel sind häufig Gegenstände des persönlichen und häuslichen Ambientes.

Wer aber möchte sich schon gerne eine Bimsmaschine oder Grubenlore in sein Wohnzimmer stellen? Auch ein präparierter Haifisch wäre nur kurzfristig ein Gag Ihrer Wohnung.

2. Erfolgreich sind Sie, wenn Ihr Personal ehrenamtlich, d.h. ohne finanzielle Gegenleistung für Sie arbeitet. Etwa 2.500 Stunden im Jahr sollte Ihr Shop geöffnet sein. Bei lediglich 10 DM pro Stunde Gehalt sind dies 25.00o DM jährlich, ohne die 20 Prozent Lohnsteuer, sieben Prozent davon an Kirchensteuer und derzeit 5,5 Prozent der Lohnsteuer als Solidaritätszuschlag - also stramme 30.850 DM. Schade um dieses Geld, das Ihnen dann fehlt. Aber wie?

Bauen Sie vielfältige Verbindungen zu Service-Clubs wie Rotary, Lions oder Zonta auf, ersatzweise auch zu wichtigen Mitgliedern Ihres Fördervereins. Damen jenseits der 45 haben ihre Kinder über die Schulhürden gebracht, haben einen Gatten mit sattem Einkommen und einem social standing, das der Ehefrau eine regelmäßige Tätigkeit zum Broterwerb untersagt. Wie hilfreich bietet sich da Ihr Shop an, vor allem, wenn er sich kulturell anspruchsvoll zeigt. Leider haben wir ein solches kulturelles und soziales Engagement in Deutschland nach dem Zweiten Weltkrieg weitgehend verlernt, im Gegensatz zu den Vereinigten Staaten, wo häufig in größeren Museen genau so viele »Volunteers« wie Angestellte arbeiten.

3. Kaufen Sie vor allem vorsichtig ein. 15.000 oder 20.000 DM sind schnell investiert. Wenn der Verkauf nicht Ihren Erwartungen entspricht, haben Sie Ihr Kapital über Gebühr gebunden. Sie manövrieren sich in die Ecke, die Sie zu Kurzschluß- 
handlungen verleiten kann. Versuchen Sie statt dessen, möglichst viele Waren in Kommission zu nehmen. Sie zahlen dann am Monatsende nur das, was Sie verkauft haben. Ihr Kapital steht Thnen noch zur Verfügung. Unsere Erfahrungen zeigen, daß erstaunlich viele Lieferanten diese Konditionen akzeptieren.

4. Die Lage Ihres Shops im Museum ist so auszuwählen, daß jeder Besucher mindestens zweimal hineinschaut und der Wunsch in ihm reift, vor dem Verlassen des Hauses noch einkaufen zu gehen. Ausgewählte Waren, gut präsentiert, wecken Neugierde.

5. Zur Dekoration des Shops sollte das Museum überlegen, welche attraktiven Exponate nicht permanent präsentiert werden und den Shop zieren könnten. Bei uns sind es Modellflugzeuge der Konstruktionen von Carl Clemens Bücker, am Fuße der Festung geboren, die zu unserer Bücker-Abteilung mit zwei Originalmaschinen gehören.

6. Von besonderer Wichtigkeit ist, daß Sie rechtzeitig mit dem Träger Ihres Museums rechtskräftige und verbindliche $\mathbf{A b}$ sprachen treffen, z.B. über die mietfreie Nutzung von Räumen und Flächen oder über die Befreiung von Reinigungs- und Energiekosten, also Kosten, die Ihre Bilanz unattraktiver machen und wahrlich überflüssig sind, da ja Thre Aktivitäten und Gewinne ausschließlich dem Museumsträger und seiner Finanzdisposition zugute kommen.

7. Bedenken Sie dabei auch immer, daß es Menschen geben kann, auch in Ihrem engeren beruflichen Umfeld, die Ihnen persönlich schaden wollen und auf vermeintliche juristische Unzulänglichkeiten warten, um Ihnen eine Grube zu graben.

8. Es wäre sicherlich falsch, würde man die Themen der Sonderausstellung eines Museums primär nach kommerziellen Erwartungen auswählen. Die Verlockung besteht dennoch, gerade wenn Sie Erfahrungen gemacht haben, die Ihnen solches nahelegen, wie man anhand einer Grafik mit den Zahlen unserer Museumsbesucher und der Shopumsätze sehen kann. 


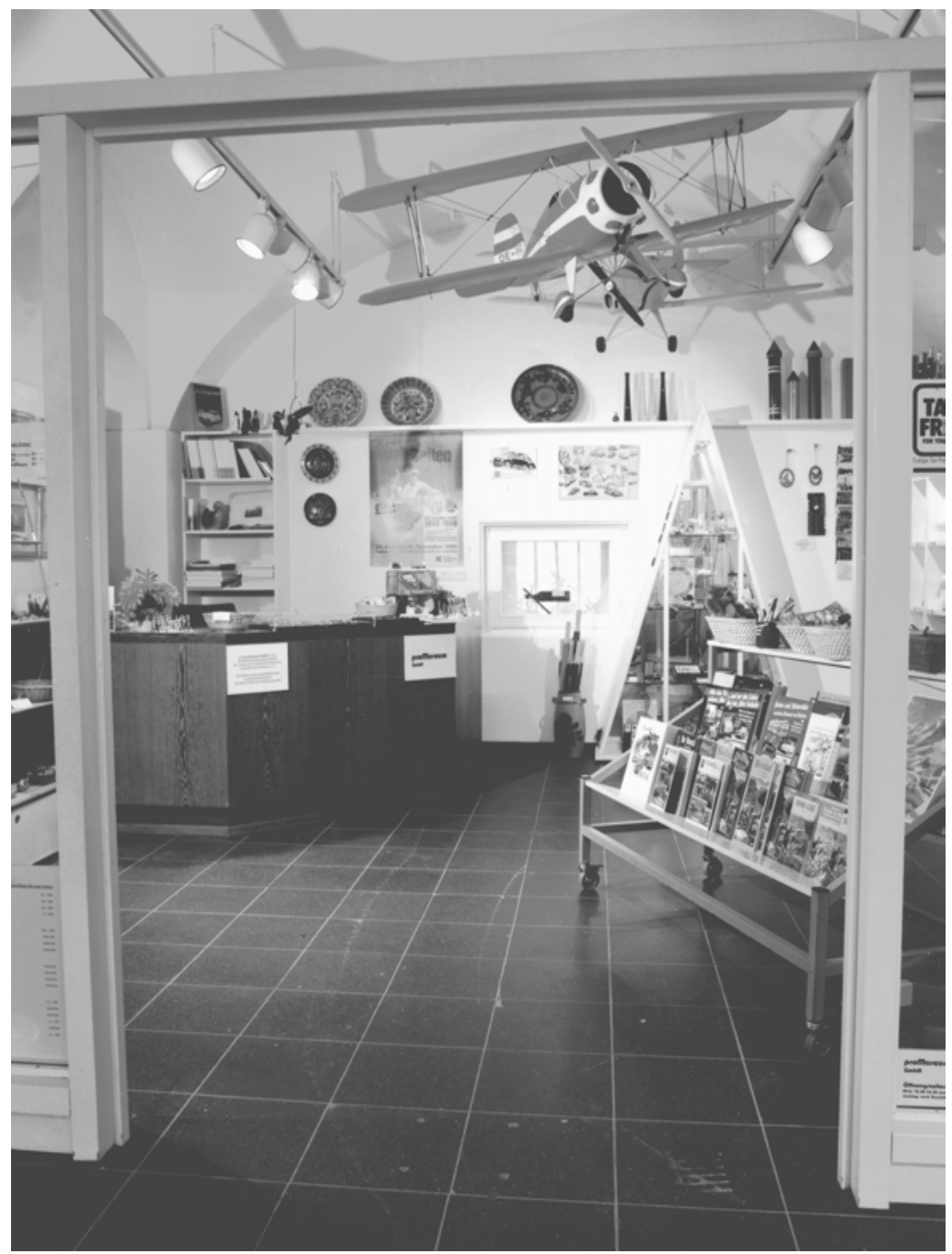

Ausgewählt habe ich die Jahre 1993 bis 1998, beginnend mit einer Zeit der noch fetten Jahre des Besucherbooms und des freien Eintritts in unser Museum. 1993 war zugleich das Gründungsjahr der proMuseum $\mathrm{GmbH}$. Besucherzahlen über 250.00o oder gar 300.00o jährlich waren keine Seltenheit bei uns. Der Abwärtstrend in der Besucherstatistik setzte bei uns zeitversetzt ein, da wir in den frühen goer Jahren für die neuen Bundesländer lohnendes Ausflugsziel (Blick auf das »Deutsche Eck«) waren. Die Shopumsätze von 132.300 DM in 1993 und 141.900 DM in 1994 mit o,44 DM 


\section{Abbildung 1}

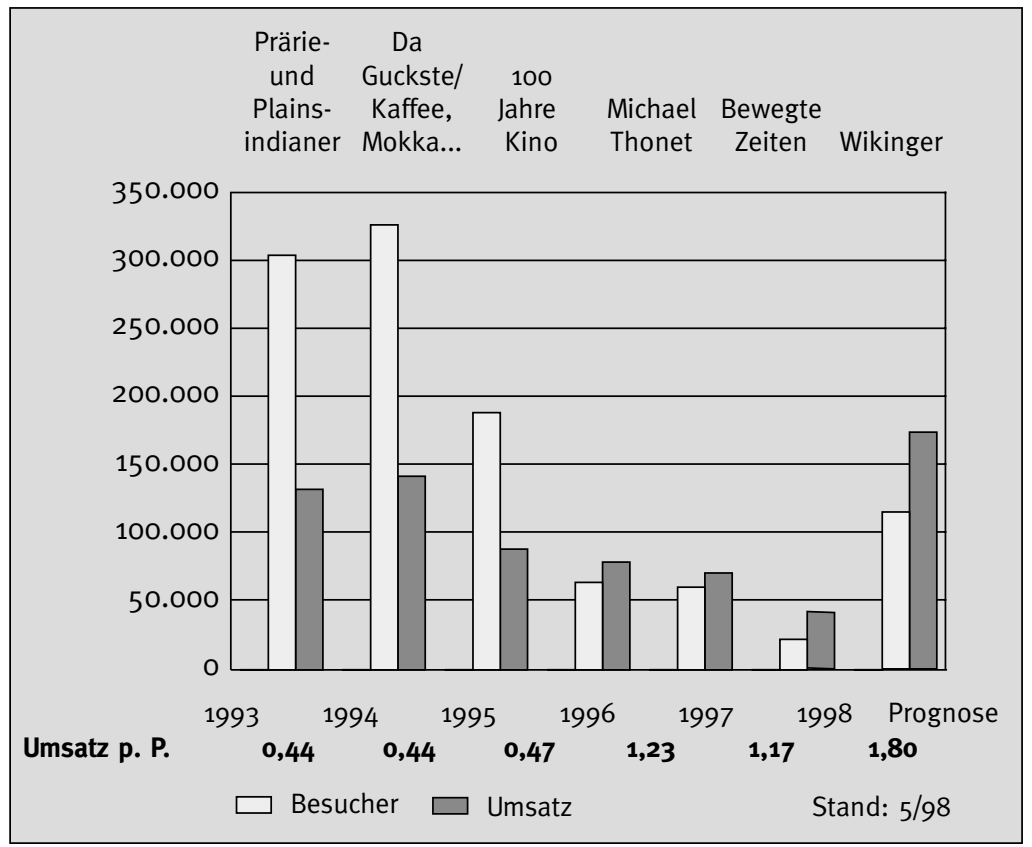

pro Besucher waren maßgeblich bestimmt durch die Sonderausstellungen. Die Prärie- und Plainsindianer brachten uns alle Indianerfans aus Deutschland und den Beneluxländern ins Haus. Navajo-Silberschmuck war der große Renner und in Deutschland noch nicht so verbreitet. Reichlich behangen zog mancher Hilfsindianer von dannen. Seltsamerweise verkaufte sich dieser im Folgejahr fast genau so gut. Es hatte sich herumgesprochen, daß er bei uns günstig erworben werden konnte. Die 94er Zahlen beinhalten auch die Umsätze eines Wiener Kaffeehauses in der Ausstellung »Kaffee-Mokka-Muckefuck. Eine Kultur- und Technikgeschichte des Kaffeetrinkens."

Der Einbruch bei den Besucherzahlen begann dann 1995. Von einem Jahr zum anderen verloren wir 42 Prozent unserer Besucher trotz einer hochattraktiven Sonderausstellung zum Thema "100 Jahre Kino". Glücklicherweise sanken die Shopumsätze nicht in gleichem Maße, sondern nur um 38 Prozent. Dafür aber erzielten wir leichte Verbesserungen bei den Pro-Kopf-Umsätzen von o,44 DM auf 0,47 DM. Die nächste Katastrophe folgte ein Jahr später, als wir nach 25 Jahren freien Eintritts 1996 erstmals wieder 
Eintritt erheben mußten. Bei lediglich 3 DM für Einzelbesucher und abgestuftem Sozialkanon verloren wir gegenüber 199566 Prozent und gegenüber 1994 gar 80,3 Prozent unserer Besucher. Wir hatten gehofft, den erneuten Besucherschwund durch eine besonders attraktive Sonderausstellung über Michael Thonet, dessen 200. Geburtstag anstand, ausgleichen zu können, was nicht gelang. Michael Thonet war offensichtlich ein Thema von allgemein geringerer Attraktivität.

Allerdings reduzierten sich die Shop-Umsätze lediglich um 10,7 Prozent, und zum ersten Male wurde mit 1,23 DM pro Besucher die 1 DM-Grenze deutlich überschritten. Das Jahr 1997 ließ uns noch einmal Besucher- und Umsatzeinbußen vermerken, und es war uns völlig unverständlich, warum die Sonderausstellung »Bewegte Zeiten. Wir werden mobil 1945-1960« mit über 50 historischen Motorfahrzeugen, Jugendträume der älteren Generation, nicht die Menschen zum Kauf motivierte. Wir müssen uns fragen, ob unser Marketing unzulänglich war.

Dieses haben wir 1998 grundlegend geändert, indem wir eine renommierte Werbeagentur mit der Öffentlichkeitsarbeit für die Hauptausstellung »Die Wikinger« beauftragten. Die dafür getätigten Investitionen haben sich nachhaltig gelohnt. Bereits am ersten Öffnungstag hatten wir 2.100 Besucher, von denen 85 Prozent nachmittags zwischen 14 und 17 Uhr kamen, so daß wir zeitweise die Ausstellung wegen Überfüllung schließen mußten. Hinzu kam aber auch, daß das Thema der Ausstellung eine vergleichbare Emotionalität auslöste, wie wir diese von der Indianerausstellung kannten.

Die ersten sechs Wochen der Ausstellung zeigten, daß der Umsatz pro Person auf 1,80 DM angestiegen ist, bedingt durch den Verkauf von Wikinger-Schmuck und Wikinger-Literatur.

Die letzte Säule zeigt den Versuch einer Hochrechnung für das Jahr 1998 auf der Basis der ersten sechs Wochen; doch müssen wir jetzt schon feststellen, daß der Besucherandrang nachläßt und wir mit neuen Werbemitteln darauf reagieren müssen.

Wir werden vielleicht das Tal, das sich auf einer anderen Grafik deutlich zeigt, überwinden, haben vielleicht auch bessere Marketingmittel gefunden.

Sie sehen, daß ein Museumsshop noch lange nicht mit etwas Goodwill, ein paar Ideen und etwas Geld funktionieren muß. Von den immer wieder zitierten 5 DM pro Kopf, die manches Kunstmuseum umsetzen will, sind wir jedenfalls noch weit entfernt. 


\section{Abbildung 2}

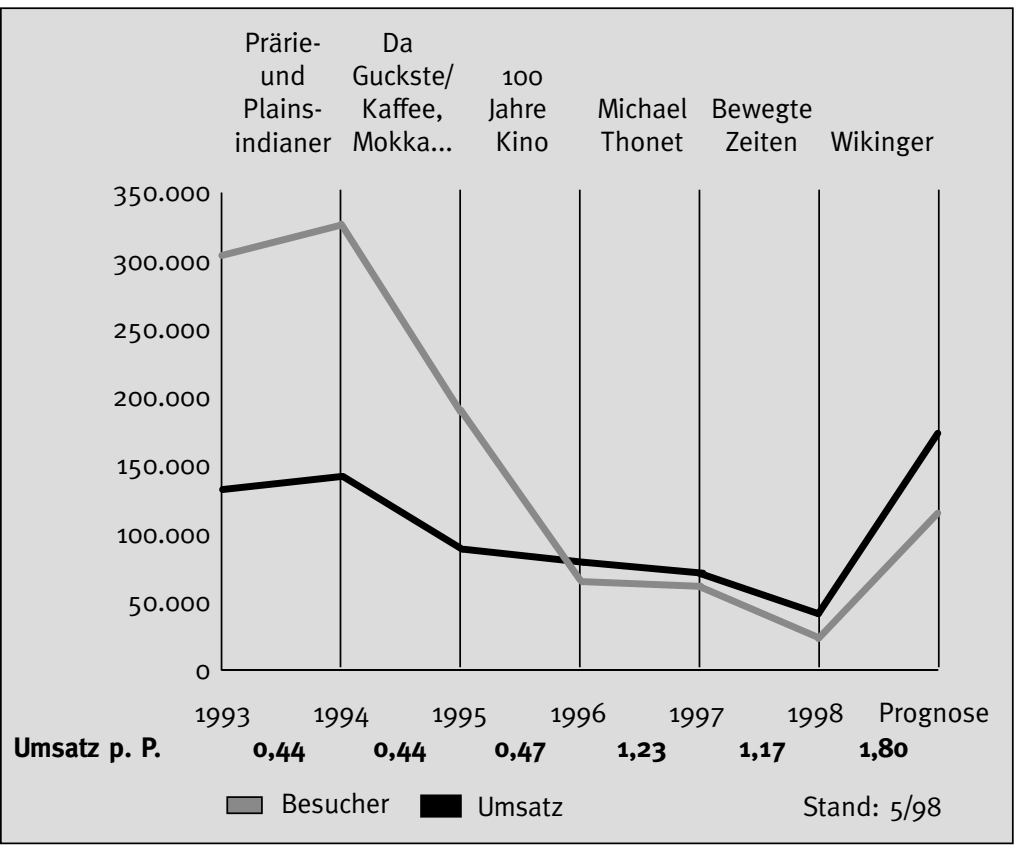

Oftmals haben wir überlegt, den Shop zu schließen. Er ist aber eines unserer wichtigen Serviceangebote an den Besucher.

Pommes frites und Curry-Würste lassen sich sicherlich ohne große Vorkenntnisse und Schulung an die Frau oder den Mann bringen, weil sich der Hunger verkaufsfördernd auswirkt. Gehen Sie aber nicht zwangsläufig davon aus, daß Sie auch der geborene Shop-Betreiber und Starverkäufer sein müssen. Sie benötigen - im Gegensatz zur Imbißbude - ein Rüstzeug, das Sie sich erst aneignen müssen. So erwartet auch der Käufer, daß Sie nicht nur über Materialien und Verarbeitungstechniken informiert sind, sondern auch inhaltlich das Objekt einordnen können; die Lektüre der Fachliteratur, die Sie im Idealfall ebenso verkaufen, sollte daher Pflicht sein. Ihr Wissen fördert den Verkauf!

Zehn mir wichtige Punkte möchte ich abschließend noch einmal festhalten; ich habe sie genannt: »Nicht vergessen!«

- Der Renner dieses Sommers kann schon im nächsten Jahr der unbegreifliche Ladenhüter sein. Dies gilt vor allem für Modeschmuck. Seien Sie auf der Hut! 
- Der Erfolg kommt selten im ersten Anlauf. Testen Sie vorsichtig Ihren Markt, korrigieren sie behutsam und schaffen Sie sich einen Beraterkreis mit Personen Ihres Vertrauens.

- Suchen Sie sich Partner, deren Rahmenbedingungen den Ihrigen gleichkommen. Gemeinsam können sie stärker sein im Einkauf, im Verkauf oder in der Aufarbeitung von Ladenhütern.

- Machen Sie sich schlau, erfahren Sie, wie es bei den Nachbarn läuft. Nicht die Kopie, das eigene Profil ist gefragt, und Sie sind der Profilgeber.

- Besuchen Sie Fachmessen, wie die Frankfurter "Ambiente«, um die Marktmöglichkeiten, die Anbieter und die Konditionen zu erfahren. Sie müssen sich Ihren Lieferanten aufdrängen, visuell bekannt machen und immer wieder präsent sein.

- Prüfen sie Kreditkartenunternehmen und lokale Kartenangebote (in Koblenz haben wir die Tourismus-Karte »KoblenzCard«) auf ihre Akzeptanz und wirtschaftliche Rentabilität, speziell für Sie!

- Haben Sie dennoch Mut zum Außergewöhnlichen, vor allem wenn dies hilft, Ihr Image als Geschenkeladen mit besonderem Profil in der Region zu festigen. Wir haben dies über Jahre geschafft. Ein Sonntag, etwa sechs Wochen vor Weihnachten, mit reduzierten Preisen für Mitglieder des Freundeskreises hilft manchem, das Problem der Weihnachtsgeschenke wenigstens teilweise zu reduzieren. Achtung: Stimmen Sie solche Preisaktionen mit Ihrer Industrie- und Handelskammer ab, damit Sie keinen Ärger bekommen!

- Achten Sie darauf, daß keine spürbare Diskrepanz zwischen dem wissenschaftlichen Standard, den Sie sich gesetzt haben, und dem Warenangebot entsteht. Vorrang muß nach wie vor unser eigentlicher wissenschaftlicher und pädagogischer Auftrag haben.

- Ein Museumsshop wird dann zur Gratwanderung, wenn er nicht ein eigenes, am Hause und an den Sammlungen orientiertes Profil zeigt. Die schnelle Mark am falschen Platz kann sich bitter rächen.

- Haben Sie dennoch den Mut des Unternehmers, der mit seinem Risiko spielt und dieses als umsatzfördernd einsetzt. Auch sie haben ein Risiko einzukalkulieren, was durchaus für Spannung sorgen kann. 
Die Jahrestagung des Deutschen Museumsbundes hat sich auch eingehend mit der veränderten Situation in unseren Museen und unserer Reaktion darauf befaßt; empfohlen werden:

- flexiblere Öffnungszeiten

- Einrichtung von Cafés

- Verkauf von Literatur und - wie einer Zeitung zu entnehmen ist - auch von Popcorn in einem Shop und

- »Events« wie »Techno-Parties«, Bierbrauen bei historischen Ausstellungen (denken Sie an unsere Backausstellung), GalaDiners für Reiche oder Happenings mit Künstlern.

Ich stimme den Kollegen bedingt zu, die eine Umwandlung unserer Museen in Rummelplätze befürchten.

Trotz allem, wofür ich in diesem Beitrag plädiert habe, teile ich auch die Meinung, daß man den Charakter des Museums als Ort der Besinnung und Auseinandersetzung mit Expositionen nicht durch falsch definiertes Marketing zerstören soll. Die Suche nach "Events«, die man uns abverlangt, darf nicht in einen Erlebniswahn münden.

Außerdem bekommen wir ja sofort die Folgen zu spüren, wie das "Museum für Arbeit und Technik« in Mannheim, dessen hohe Akzeptanz der Ausstellung »Körperwelten« zu Haushaltskürzungen der Landesmittel in Höhe von 1 Mio. DM und des Stadtzuschusses um 500.000 DM führte, so in den Tageszeitungen vom 15. Mai 1998 zu lesen.

\section{Wie geht es weiter?}

Die Geschäftsergebnisse der letzten Jahre haben einmal mehr gezeigt, daß ein positives Geschäftsergebnis nur dann zu erwarten ist,

- wenn die Personalkosten deutlich gesenkt werden können,

- die Produktpalette sich an den aktuellen Ausstellungsthemen orientiert, weil diese

- $\quad$ eine spezielle und kaufinteressierte Klientel ansprechen, und

- wenn ein Verbund mit anderen Museumsshops geschaffen wird, der günstigere Einkaufskonditionen und eine Weitergabe von Artikeln an andere Shops ermöglicht. 


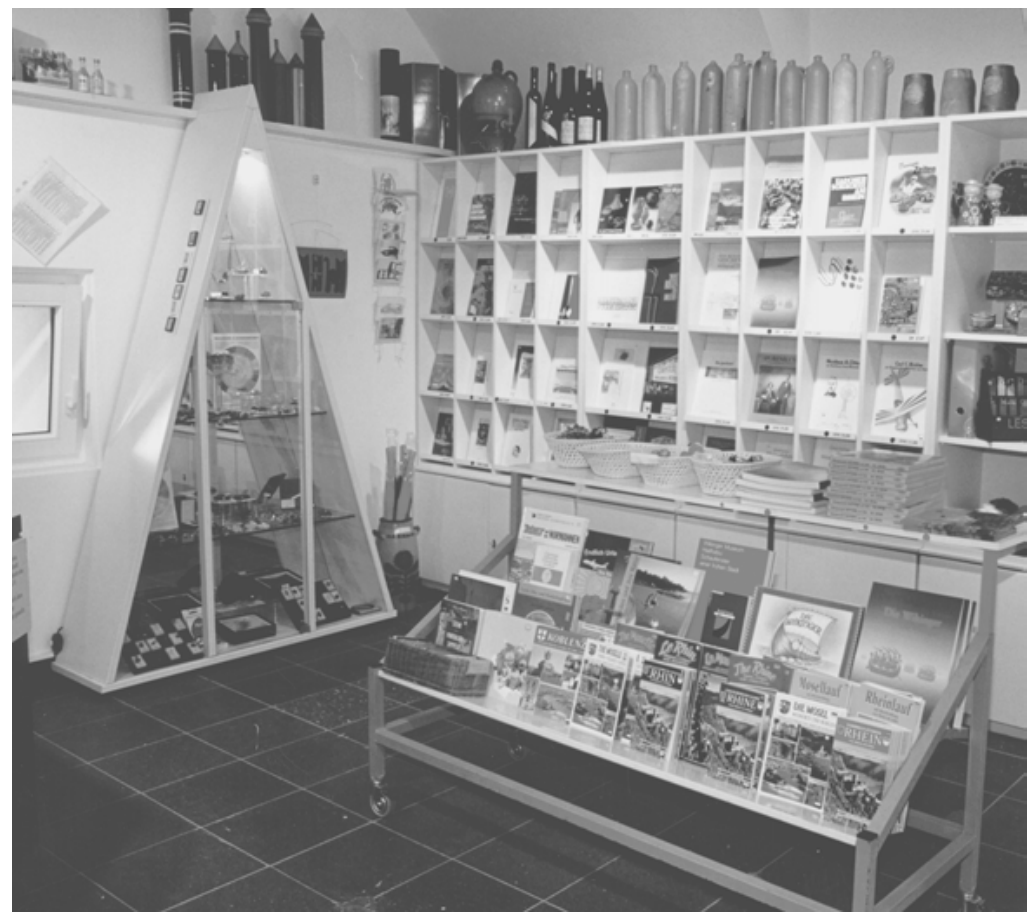

Die Geschäftsergebnisse der Jahre 1997 und 1998 rieten zu einer Vergabe der Aktivitäten an professionellere Hände, um die Risiken zu minimieren. Ausgewiesene, langjährige Betreiber mehrerer Museumsshops schienen geeignete Partner zu sein. Wir glauben, einen solchen gefunden zu haben; der vertragliche Abschluß steht derzeit (Januar 1999) unmittelbar bevor. Die mittel- bzw. langfristige Zusicherung einer zehnprozentigen Umsatzbeteiligung entspricht den Geschäftsergebnissen der letzten Jahre und befreit den früheren Betreiber von zahlreichen Problemfeldern, die zukünftig nicht mehr belasten und zeitlich binden. Freiräume für andere Aktivitäten werden wieder verfügbar.

Wichtig ist, daß der neue Geschäftspartner

- über umfangreiche Erfahrungen im Betreiben von Museumsshops verfügt, denn nicht zuletzt ist eine erhöhte Professionalität im Markt für alle Seiten vorteilhaft; 
- Warenangebote dahin translozieren kann, wo diese gefragt sind, denn 'Ladenhüter eines Shops lassen sich in einem anderen möglicherweise besser vermarkten;

- über bessere Einkaufskonditionen verfügt, die eine preisgünstigere Vermarktung ermöglichen.

Ein weiterer Vorteil eines neuen Geschäftspartners ist, daß sich steuerrechtliche Konsequenzen besser kalkulieren lassen.

Wie sich diese Erfahrungen mittel- und langfristig für unser Museum auswirken werden, kann Gegenstand späterer Berichte sein. Telefonische Zwischenberichte können unter der Telefonnummer (0261) 97 03-o gegeben werden. Da der Betrieb eines $\mathrm{Mu}-$ seumsshops aus meiner Sicht ein permanent dynamischer Prozeß ist, sollte der Erfahrungsaustausch zu unser aller Nutzen gesehen werden.

\section{Anmerkung}

* Die Aktivitäten vor Gründung der proMuseum GmbH wurden vom Autor in den folgenden Publikationen ausführlicher dargestellt und können dort nachgelesen werden:

Löber, Ulrich (1993): „Alternative GmbH?! Die Gründung einer $\mathrm{GmbH}$ am Beispiel des Landesmuseums Koblenz«. Mitteilungsblatt des Museumsverbandes Niedersachsen/Bremen 46, S. 41-56.

Löber, Ulrich (1996): »Festung Ehrenbreitstein - ein Landesmuseum mit angeschlossener GmbH«. In: Annette Zimmer (Hg.): Das Museum als Nonprofit-Organisation - Management und Marketing, Frankfurt/Main, New York, S. 443-453. 



\section{Anhang}





\section{Die Autoren}

Antonius Dommers, Fachhochschulstudium zum Verwaltungswirt; seit 1972 Leiter der Verlag- und Betriebsgesellschaft des Landschaftsverbandes Rheinland $\mathrm{mbH}$, Abtei Brauweiler in Pulheim.

Univ.-Prof. Dr. Bernd Günter, Studium der Wirtschaftswissenschaften; 1979 - 1989 Geschäftsführer des Instituts für Unternehmensführung und Unternehmensforschung der Ruhr-Universität Bochum; 1989 - 1991 Professor für Allgemeine Betriebswirtschaftslehre, FU Berlin; seit 1991 Professor für Betriebswirtschaftslehre, insbesondere Marketing, Wirtschaftswissenschaftliche Fakultät, Heinrich-Heine-Universität Düsseldorf.

Eva Hoffmeister, Kulturmanagement-Studium an der Fernuniversität - Gesamthochschule Hagen; seit Anfang der goer Jahre in der Rheinland-Verlag- und Betriebsgesellschaft $\mathrm{mbH}$ im Bereich "Besucherservice von Museen" tätig, jetzt freiberufliche Kulturmanagerin mit Schwerpunkt "kommerzielle Warenangebote/ Kulturmerchandising«.

Dr. Hans Walter Hütter, Studium von Latein, Geschichte und Pädagogik; Leiter der Abteilung Öffentlichkeitsarbeit, Museumspädagogik, Medien und stellvertretender Direktor am Haus der Geschichte der Bundesrepublik Deutschland in Bonn. 
Dr. Hartmut John, Studium der Geschichtswissenschaft, Politik und Wirtschaftsgeographie; Leiter der Abteilung "Museumsberatung « und des Fortbildungszentrums Abtei Brauweiler/ Rheinisches Archiv- und Museumsamt des Landschaftsverbandes Rheinland.

Dr. Ulrich Löber, Studium der Volkskunde, Germanistik, Vorund Frühgeschichte und Soziologie; seit 1976 Direktor des Landesmuseums Koblenz - Staatliche Sammlung technischer Kulturdenkmäler.

Claus-Peter Pithan, Studium an der Fachhochschule für Finanzen in Nordrhein-Westfalen; Mitarbeiter in der Kämmerei des Landschaftsverbandes Rheinland; Mitglied der Arbeitsgruppe "Steuerpflicht kommunaler Kultureinrichtungen« des Deutschen Städtetags.

Dr. Gabriele Uelsberg, Studium der Kunstgeschichte, Archäologie sowie Ur- und Frühgeschichte; seit 1994 Leiterin des Städtischen Museums in Mülheim a.d.Ruhr; Werkleiterin im Eigenbetrieb Kultur der Stadt Mülheim a.d. Ruhr.

Dipl.-Ing. Brigitte Voswinkel, Studium zur Diplom-Ingenieurin; Technisches Assistentin an der Bergischen Universität Wuppertal, Fachbereich Physik; seit 1990 zunächst maßgeblich beim Aufbau des Museumsshops im Von der Heydt-Museum Wuppertal beteiligt; danach ehrenamtlich im Shopmanagement tätig und u.a. zuständig für Marketing / Produktentwicklung. 


\section{Bildnachweis}

S. 3: Compania Media

S. 18, 20, 25, 27: Haus der Geschichte der Bundesrepublik

Deutschland, Bonn/Fotostudio Querbach

S. 61, 63, 67: Von der Heydt-Museum, Wuppertal/Museums Shop

Organisation

S. 95, 96, 97, 98, 99: Rheinland-Verlag- und Betriebsgesellschaft, Puhlheim

S. 105, 106, 109: Städtisches Museum Mühlheim/Ruhr

S. 116, 122: proMuseum GmbH, Koblenz 


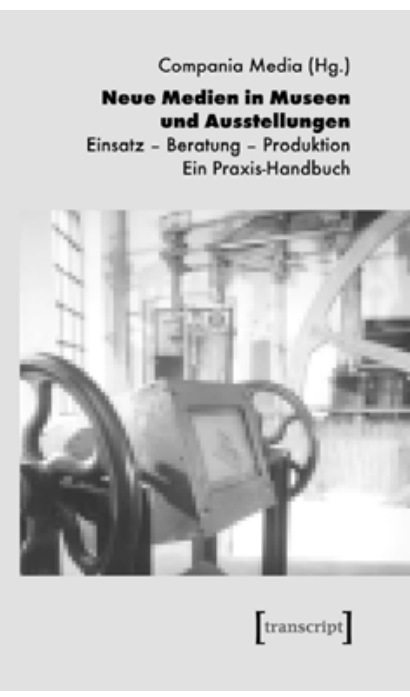

Compania Media (Hg.)

Neue Medien in Museen

und Ausstellungen

Einsatz - Beratung - Produktion Ein Praxis-Handbuch

mit Interviews und Beiträgen von (u.a.):

Dr. Bernhard Graf, Ulrike Pohler,

Wolfgang Röhrig, Jörg Schmidtsiefen 1998, 516 Seiten, ca. 100 Abb., kart., 98 DM ISBN 3-933127-00-9
Das vorliegende Praxis-Handbuch bietet u.a. folgende Informationen:

- Der Teil »Neue Medien im Einsatz bietet auf 250 Seiten die Analyse von acht Besucherinformationssystemen, $15 \mathrm{CD}$-ROMs und 12 Web-Sites aus 29 deutschen Museen. Behandelt werden u.a. die Integration von Medien in Ausstellungskonzepte und -design, die praktische Organisation und die finanzielle Seite der Produktion, auftretende Probleme und individuelle Lösungsstrategien.

- Drei Expertenbeiträge zu den Themen: "Kooperation von Museen und Medienproduzenten «, "Neue Medien und Recht" und »Technische Voraussetzungen für den Zugang zum Internet" und zwei Interviews bereichern den Informationsbestand um praxisrelevantes Sachwissen.

- Der ca. 20o Seiten umfassende "Einrichtungs- und Branchenführer « macht das Leistungsspektrum von 43 an der Schnittstelle zwischen Museum und Neuen Medien tätigen Einrichtungen des Museumswesens transparent. Die detaillierte Darstellung von 22 einschlägig ausgewiesenen Beratern und 83 Produktionsfirmen bzw. -einrichtungen unterstützt Museen dabei, geeignete Partner zur Realisierung von Projekten im Bereich Neuer Medien zu finden.

Das Praxishandbuch richtet sich an Museumsmitarbeiter, Ausstellungsgestalter, Produzenten, Berater, Bildungsinstitutionen, Pädagogen, Kulturmanager, Journalisten, Studierende und Museumsinteressierte. 


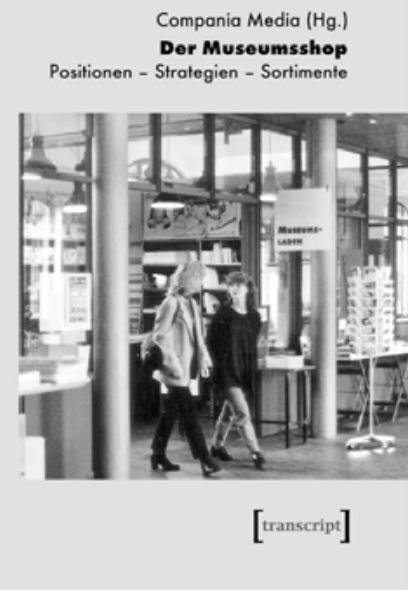

Compania Media (Hg.)

\section{Der Museumsshop}

Positionen-Strategien-Sortimente Ein Praxisführer

mit Interviews und Beiträgen von (u. a.): Hans-Jürgen Brockmeyer, Alexander Fietz, Carole Gürtler, Walter Hofmann, Jörg Inter, Mario Leupold, Hanno Rauterberg, Henning Schaper, Walter Scheuerl, Harald Siebenmorgen, Ute-Maria Sunkel, Brigitte Voswinkel und white balance 1999, 384 Seiten, ca. 100 Abb., kart., 78 DM ISBN 3-933127-02-5
Der Praxisführer bietet folgende Informationen:

- Im ersten Teil des Buches beleuchten Experten unterschiedliche Aspekte des Themas "Shoppen im Museum«. Auf ca. 120 Seiten werden u. a. die rechtlichen und organisatorischen Implikationen von Museumsshops, Fragen der Integration des Shops ins Museum und des Sortiments behandelt. Erfahrungsberichte aus fünf Museen komplettieren den Informationsbestand.

- Der zweite Teil präsentiert elf Fallbeispiele aus verschiedenen Segmenten der Museumslandschaft im deutschsprachigen Raum, wobei unterschiedliche Museumstypen und -größen und insbesondere auch die spezifische Situation der kleineren Museen Berücksichtigung finden. Die verschiedenen Modelle, Strategien und Konzepte werden auf ca. 150 Seiten ausführlich vorgestellt. Praxistips der interviewten Museumsmitarbeiter und Shopbetreiber bilden den Abschluß des zweiten Teils.

- Der dritte Teil bietet einen Branchenführer mit detaillierten Informationen zu Produzenten und Anbietern von Artikeln und Dienstleistungen für Museumsshops. Dieser hilfreiche Führer umfaßt ca. 100 Seiten.

Die Veröffentlichung richtet sich an Museumsmitarbeiter, Shopbetreiber und -mitarbeiter, Produzenten, Berater, Bildungsinstitutionen, Kulturmanager, Journalisten und Museumsinteressierte. 


\section{Fortbildungs-}

\section{zentrum}

\section{Abteilung Museumsberatung und Fortbildungszentrum Abtei Brauweiler des Rheinischen Archiv- und Museumsamtes}

Abtei

Brauweiler

\section{Schriften des Rheinischen Museumsamtes:}

Nr. 57 Neue Strukturen für Museen?

Tagungsband zum gleichnamigen Kolloquium 1993

Rheinland-Verlag, Pulheim 1994,

160 S., Abb., kart., 14,80 DM
Nr. 61 Vom Elfenbeinturm zur Fußgängerzone Tagungsband zum gleichnamigen Kolloquium 1994 Verlag Leske + Budrich, Opladen 1996, 204 S., kart., 28,00 DM

\section{Publikationen der Abteilung Museumsberatung:}

Nr. 1 Das besucherorientierte Museum

Tagungsband zum gleichnamigen Kolloquium 1995

Rheinland-Verlag, Pulheim 1997,

139 S., Abb., kart., 18,00 DM

Nr. 2 Dem «Zahn der Zeit» entrissen!

Neue Forschungen und Verfahren zur

Schädlingsbekämpfung im Museum

Rheinland-Verlag, Pulheim 1997,

186 S., Abb., kart., 25,00 DM

Nr. 3 Der Salamander - ein gar fürchterliches Thier Zur Natur- und Kulturgeschichte des Feuersalamanders Katalog zur gleichnamigen Ausstellung

Rheinland-Verlag, Pulheim 1997,

96. S., Abb., kart., 25,00 DM

Nr. 4 Zum Bedeutungswandel der Kunstmuseen.

Positionen und Visionen zu Inszenierung,

Dokumentation, Vermittlung

Tagungsband zum gleichnamigen Kolloquium 1996

Verlag für moderne Kunst, Nürnberg 1998,

198 S., Abb., kart., 45,00 DM

Nr. 5 Süßes Rheinland

Zur Kulturgeschichte des Zuckers

Katalog zur gleichnamigen Ausstellung 1998

Bouvier Verlag, Bonn 1998,

120 S., Abb., kart., 24,80 DM

Nr. 6 „Farbfehler! « Gegen das Verschwinden der Farbfotografie

Tagungsband zum gleichnamigen Kolloquium 1997

Verlag Rundbrief Fotografie, Sonderheft Nr. 5 ,

Stuttgart 1998, 200 S., Abb., kart., 29,50 DM

\section{Nr. 7 Die Lust zu Wohnen - \\ Das Rote Haus in Monschau \\ Wienand Verlag, Köln 1998,}

190 S., Abb., kart., 49,00 DM

Nr. 8 Shops und kommerzielle Warenangebote Tagungsband zur gleichnamigen Veranstaltung des Fortbildungszentrums Abtei Brauweiler 1998 transcript Verlag, Bielefeld 2000,

130 S., Abb., kart., 39,80 DM

Nr. 9 Besucher zu Stammgästen machen! Neue und kreative Wege zur Besucherbindung Tagungsband zum gleichnamigen Kolloquium des Fortbildungszentrums Abtei Brauweiler 1998 transcript Verlag, Bielefeld, erscheint Sommer 2000, ca. 180 S., kart., ca. 40 DM

\section{Nr. 10 euphorie digital?}

Aspekte der Wissensvermittlung in Kunst, Kultur und Technologie

Tagungsband zum gleichnamigen Kolloquium 1998 des Fortbildungszentrums Abtei Brauweiler und des Heinz Nixdorf MuseumsForums Paderborn transcript Verlag, Bielefeld, erscheint Sommer 2000, ca. 180 S., kart., ca. 40 DM

Nr. 11 Museen und Sammlungen im Rheinland (Museumsführer) erscheint 2000

Bestelladresse: Landschaftsverband Rheinland, Rheinisches Archiv- und Museumsamt Abteilung Museumsberatung und Fortbildungszentrum Abtei Brauweiler Ehrenfriedstraße 19, D-50259 Pulheim Tel.: 02234/9854-302 oder -313, Fax: 02234/9854-202 e-mail: t.martini@mail.lvr.de, Internet: http://www.lvr.de

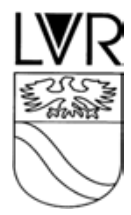

